POTENTIAL THEORY METHODS FOR SOME NONLINEAR ELLIPTIC EQUATIONS

\author{
A Dissertation \\ presented to \\ the Faculty of the Graduate School \\ at the University of Missouri \\ In Partial Fulfillment \\ of the Requirements for the Degree \\ Doctor of Philosophy \\ by \\ Adisak Seesanea \\ Dr. Igor E. Verbitsky, Dissertation Supervisor
}

July 2018 
(c) Copyright by Adisak Seesanea 2018

All Right Reserved 
The undersigned, appointed by the Dean of the Graduate School, have examined the dissertation entitled

\section{POTENTIAL THEORY METHODS FOR SOME NONLINEAR ELLIPTIC EQUATIONS}

presented by Adisak Seesanea, a candidate for the degree of Doctor of Philosophy of Mathematics, and hereby certify that in their opinion it is worthy of acceptance.

Professor Igor E. Verbitsky

Professor Tanya Christiansen

Professor Loukas Grafakos

Professor David Retzloff 
Dedicated to the memory of my mother

Yupa Seesanea 


\section{ACKNOWLEDGEMENTS}

First and foremost, I would like to express my sincere gratitude to my supervisor, Professor Igor E. Verbitsky, for introducing me to this research area, initiating fruitful discussions, and providing me invaluable opportunities, throughout my study.

I would like to extend my appreciation to the mathematicians who have taught and inspired me during my graduate journey, including Professors Tanya Christiansen, Fritz Gesztesy, Loukas Grafakos, Sergey Meleshko, Dorina Mitrea, Marius Mitrea, Peter Pivovarov, Eckart Schulz and Samuel Walsh.

I also thank Professor David Retzloff for his interest and serving as a member of my doctoral committee.

In addition, I am grateful to the Development and Promotion of Science and Technology Talents Project (DPST) in Thailand for its financial support.

Finally, I thank my family for their love and encouragement. 


\section{Table of Contents}

ACKNOWLEDGEMENTS

ABSTRACT $\quad$ v

1 Introduction 1

1.1 Overview and main results . . . . . . . . . . . . . . . . . . 1

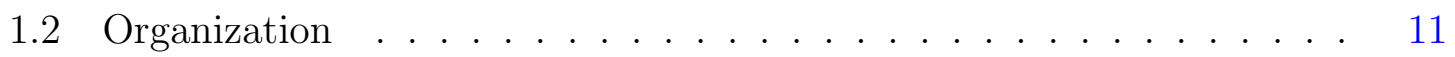

2 Preliminaries $\quad 12$

2.1 Function spaces . . . . . . . . . . . . . . . . . 12

2.2 Riesz potentials . . . . . . . . . . . . . . . . 14

2.3 Wolff potentials and energy estimates . . . . . . . . . . . 15

$2.4 \mathcal{A}$-superharmonic functions . . . . . . . . . . . . . . . . . . 17

2.5 Notion of solutions . . . . . . . . . . . . . . . . . . 19

2.6 Kernels and potentials . . . . . . . . . . . . . . . . . . . . . . 21

2.7 Some known results . . . . . . . . . . . . . . . . . . . . . 23

3 Finite energy solutions $\quad 31$

3.1 Existence result for equation $(1.1) \ldots \ldots . \ldots . \ldots . \ldots 31$

3.2 Existence result for equation $(1.2) \ldots \ldots . \ldots$. . . . . . . 40 
3.3 Existence result for equation $(1.3) \ldots \ldots \ldots$. . . . . . . 45

3.4 Uniqueness results . . . . . . . . . . . . . . . . . . . . 50

4 Generalized energy of measures $\quad 55$

5 Solutions with finite generalized energy 65

5.1 Existence result for equation $(1.3) \ldots \ldots . \ldots 65$

5.2 Proofs of Corollaries 1.4 and $1.5 \ldots \ldots$. . . . . . . . . 73

$\begin{array}{ll}\text { BIBLIOGRAPHY } & 75\end{array}$

$\begin{array}{ll}\text { VITA } & 79\end{array}$ 
Potential Theory Methods for Some Nonlinear Elliptic Equations

Adisak Seesanea

Dr. Igor E. Verbitsky, Dissertation Supervisor

\begin{abstract}
This dissertation presents a unified approach via potential theory for solvability of a class of nonlinear elliptic equations.
\end{abstract}

We give necessary and sufficient conditions for the existence of a positive finite energy solution to the quasilinear elliptic equation

$$
-\Delta_{p} u=\sigma u^{q}+\mu \quad \text { in } \mathbb{R}^{n}
$$

in the sub-natural growth case $0<q<p-1$, where $\Delta_{p}$ is the $p$-Laplacian with $1<p<\infty$, and $\sigma, \mu$ are nonnegative measurable functions (or measures) on $\mathbb{R}^{n}$. Uniqueness of such a solution in certain Sobolev space is demonstrated as well.

A similar problem in the sublinear case $0<q<1$ is treated for the fractional Laplace operator $(-\Delta)^{\alpha}$ with $0<\alpha<\frac{n}{2}$, in place of $-\Delta_{p}$ on $\mathbb{R}^{n}$.

In the classical case $\alpha=1$, we obtain the existence result for positive solutions with finite generalized energy: $\mathbb{E}_{\gamma}[u]:=\int_{\Omega}|\nabla u|^{2} u^{\gamma-1} d x<\infty$, for $0<\gamma<\infty$, to the corresponding sublinear elliptic equation on an arbitrary domain $\Omega \subseteq \mathbb{R}^{n}$ which possesses a positive Green function. When $0<\gamma \leq 1$, this yields sufficient conditions for the existence of a positive solution to the same problem in the Dirichlet space $\dot{W}_{0}^{1, p}(\Omega)$ for $1<p \leq 2$. Uniqueness of a finite energy solution $(\gamma=1)$ is proved.

Our approach is applicable to the problems with more general quasilinear elliptic $\mathcal{A}$-Laplace operators on $\mathbb{R}^{n}$, as well as linear uniformly elliptic operators with bounded measurable coefficients on domains $\Omega \subseteq \mathbb{R}^{n}$ which admit positive Green functions. 


\section{Chapter 1}

\section{Introduction}

The main objective of this work is to study a class of nonlinear elliptic equations. This chapter provides an overview, statements of main results and their consequences, together with the organization of this study.

\subsection{Overview and main results}

We consider the quasilinear elliptic equation

$$
-\Delta_{p} u=\sigma u^{q}+\mu \quad \text { in } \mathbb{R}^{n}
$$

in the sub-natural growth case $0<q<p-1$.

Here $\Delta_{p} u:=\operatorname{div}\left(|\nabla u|^{p-2} \nabla u\right)$ is the $p$-Laplacian with $1<p<\infty$, and $\sigma, \mu$ are nonnegative locally integrable functions on $\mathbb{R}^{n}$, or more generally, nonnegative locally finite Borel measures on $\mathbb{R}^{n}$ such that $\sigma \not \equiv 0$. Therefore rough and highly oscillating coefficients and data are considered here.

Such equation arises in many physical problems with nonlinear sources (fluid flows, heat transfers, etc.), which has been used in various fields such as Control Theory, Differential Geometry and Astronomy [Li]. For example, it corresponds to the study of the porous medium equation [BO, Vaz], and the Thomas-Fermi Theory where $\mu$ 
is a finite sum of electric pointwise charges, see $[\mathrm{BB}, \mathrm{P}]$ and literature cited there.

Simultaneously, both homogeneous $(\mu \equiv 0)$ and inhomogeneous equations $(\mu \not \equiv 0)$ will be studied here. Even though the former case was examined earlier in [CV1], investigating the problems with general data $\mu \geq 0$ involves nontrivial questions regarding possible interaction between $\mu$ and $\sigma$.

We establish necessary and sufficient conditions on $\sigma$ and $\mu$ for the existence of a positive finite energy solution $u \in \dot{W}_{0}^{1, p}\left(\mathbb{R}^{n}\right)$, so that $\int_{\mathbb{R}^{n}}|\nabla u|^{p} d x<+\infty$, to (1.1) (see Definition 2.1), and prove its uniqueness.

Our approach is also applicable to the existence problem for positive finite energy solutions $u \in \dot{H}^{\alpha}\left(\mathbb{R}^{n}\right)$, so that $\int_{\mathbb{R}^{n}}\left|(-\Delta)^{\frac{\alpha}{2}} u\right|^{2} d x<+\infty$ (see Definition 3.8), to the corresponding fractional Laplace equation

$$
(-\Delta)^{\alpha} u=\sigma u^{q}+\mu \quad \text { in } \mathbb{R}^{n}
$$

where $0<q<1$ and $(-\Delta)^{\alpha}$ is the fractional Laplacian with $0<\alpha<\frac{n}{2}$. Uniqueness of such a solution is proved in the case $0<\alpha \leq 1$.

In the classical case $\alpha=1$, or more generally, the corresponding sublinear equation

$$
\mathcal{L} u=\sigma u^{q}+\mu \quad \text { in } \Omega
$$

where $0<q<1$ and $\Omega \subseteq \mathbb{R}^{n}$ is an arbitrary domain (nonempty open connected set) which possesses a positive Green function.

The operator $\mathcal{L} u:=-\operatorname{div}(\mathcal{A} \nabla u)$ with bounded measurable coefficients is assumed to be uniformly elliptic, i.e., $\mathcal{A}: \Omega \rightarrow \mathbb{R}^{n \times n}$ is a real symmetric matrix-valued function on $\Omega$, and there exist positive constants $m \leq M$ such that

$$
m|\xi|^{2} \leq \mathcal{A}(x) \xi \cdot \xi \leq M|\xi|^{2}
$$


for almost every $x \in \Omega$ and every $\xi \in \mathbb{R}^{n}$.

Our method is employed to obtain necessary and sufficient conditions on $\sigma$ and $\mu$ for the existence of a positive $\mathcal{A}$-superharmonic solution (see Definition 3.13) $u \in$ $L_{l o c}^{q}(\Omega, d \sigma)$ with zero boundary values to (1.3), which has finite generalized energy:

$$
\mathbb{E}_{\gamma}[u]:=\int_{\Omega}|\nabla u|^{2} u^{\gamma-1} d x<+\infty, \quad \gamma>0 .
$$

In the case $\gamma=1$, we show that such a solution $u \in \dot{W}_{0}^{1,2}(\Omega)$ (the so-called finite energy solution) is unique.

When $0<\gamma<1$, this result yields sufficient conditions for the existence of a positive solution $u \in \dot{W}_{0}^{1, p}(\Omega)$ to $(1.3)$ where $1<p<2$.

We observe that the existence of positive weak solutions to homogeneous equation (1.3) with $\mu \equiv 0$, not necessarily of finite energy, is discussed in [QV1, QV2]. Further, the existence and uniqueness of bounded solutions to (1.3) on $\Omega=\mathbb{R}^{n}$ in the case $\mu$ is a nonnegative constant was characterized in [BK].

As was mentioned above, this work has been motivated by the results of Cao and Verbitsky [CV1], who showed that there exists a unique positive finite energy solution $u \in L_{l o c}^{q}\left(\mathbb{R}^{n}, d \sigma\right) \cap \dot{W}_{0}^{1, p}\left(\mathbb{R}^{n}\right)$ to the homogeneous equation

$$
-\Delta_{p} u=\sigma u^{q} \quad \text { in } \mathbb{R}^{n}
$$

where $0<q<p-1,1<p<\infty$ and $\sigma \in \mathcal{M}^{+}\left(\mathbb{R}^{n}\right)$ with $\sigma \not \equiv 0$, if and only if

$$
\mathbf{W}_{1, p} \sigma \in L^{\frac{(1+q)(p-1)}{p-1-q}}\left(\mathbb{R}^{n}, d \sigma\right)
$$

Here, for $1<p<\infty, 0<\alpha<\frac{n}{p}$ and $\omega \in \mathcal{M}^{+}\left(\mathbb{R}^{n}\right)$, the (homogeneous) Wolff potential $\mathbf{W}_{\alpha, p} \sigma$ is defined by [HW]

$$
\mathbf{W}_{\alpha, p} \omega(x)=\int_{0}^{\infty}\left[\frac{\omega(B(x, r))}{r^{n-\alpha p}}\right]^{\frac{1}{p-1}} \frac{d r}{r}, \quad x \in \mathbb{R}^{n}
$$


where $B(x, r)=\left\{y \in \mathbb{R}^{n}:|x-y|<r\right\}$ is a ball centered at $x \in \mathbb{R}^{n}$ of radius $r>0$. Notice that $\mathbf{W}_{\alpha, p} \omega=+\infty$ for $\alpha \geq \frac{n}{p}$ unless $\omega \equiv 0$. (See [AH, KuMi] for an overview of Wolff potentials and their applications in Analysis and PDE.)

For $1 \leq p<\infty$ and a nonempty open set $\Omega \subseteq \mathbb{R}^{n}$, by $\dot{W}_{0}^{1, p}(\Omega)$ we denote the homogeneous Sobolev (or Dirichlet) space defined [HKM, MZ] as the closure of $C_{0}^{\infty}(\Omega)$ with respect to the (semi)norm

$$
\|u\|_{\dot{W}_{0}^{1, p}(\Omega)}=\|\nabla u\|_{L^{p}(\Omega)}
$$

We denote by $W^{-1, p^{\prime}}(\Omega)=\left[\dot{W}_{0}^{1, p}(\Omega)\right]^{*}$ the dual space, where $p^{\prime}=\frac{p}{p-1}$ is the Hölder conjugate of $p$. If $p<n$ then $W^{-1, p^{\prime}}(\Omega) \subset \mathcal{D}^{\prime}(\Omega)$.

For equation (1.1) on $\mathbb{R}^{n}$, we will show that condition (1.7), combined with the natural assumption that $\mu$ has finite energy, i.e. (see [AH], Sec. 4.5),

$$
\mu \in \dot{W}^{-1, p^{\prime}}\left(\mathbb{R}^{n}\right) \Longleftrightarrow \int_{\mathbb{R}^{n}} \mathbf{W}_{1, p} \mu d \mu<+\infty
$$

is necessary and sufficient for the existence of a positive finite energy solution to (1.1). More precisely, we state our main results as follows.

Theorem 1.1. Let $1<p<n, 0<q<p-1$, and let $\sigma, \mu \in \mathcal{M}^{+}\left(\mathbb{R}^{n}\right)$ such that $\sigma \not \equiv 0$. Then there exists a positive finite energy solution $u \in L_{l o c}^{q}\left(\mathbb{R}^{n}, d \sigma\right) \cap \dot{W}_{0}^{1, p}\left(\mathbb{R}^{n}\right)$ to equation (1.1) if and only if both (1.7) and (1.8) hold. Moreover, such a solution is unique in $\dot{W}_{0}^{1, p}\left(\mathbb{R}^{n}\right)$. In the case $p \geq n$, there is only a trivial supersolution.

We sketch our method of proof of Theorem 1.1 in the inhomogeneous case $\mu \not \equiv$ 0 . (The homogeneous case $\mu \equiv 0$ is simpler since possible interaction between the nonlinear term involving $\sigma$ and $\mu$ is omitted.) 
We show that if (1.7) holds, then (1.8) implies a crucial two-weight condition

$$
\mathbf{W}_{1, p} \mu \in L^{1+q}\left(\mathbb{R}^{n}, d \sigma\right)
$$

which turns out to be necessary for the existence of a positive solution $u \in L_{l o c}^{q}\left(\mathbb{R}^{n}, d \sigma\right) \cap$ $\dot{W}_{0}^{1, p}\left(\mathbb{R}^{n}\right)$ to $(1.1)$

Given (1.7), this allows us to deduce the existence of a positive finite energy solution $u$ to equation (1.1) under assumption (1.8), by using a positive solution $\tilde{u} \in L^{1+q}\left(\mathbb{R}^{n}, d \sigma\right)$ to the corresponding nonlinear integral equation

$$
\tilde{u}=\mathbf{W}_{1, p}\left(\tilde{u}^{q} d \sigma\right)+\mathbf{W}_{1, p} \mu \quad \text { in } \mathbb{R}^{n}
$$

Such a solution $\tilde{u}$ can be constructed by an iterative method, provided (1.9) holds.

As shown in [COV1], condition (1.7) is equivalent to the trace inequality

$$
\|\varphi\|_{L^{1+q}\left(\mathbb{R}^{n}, d \sigma\right)} \leq C\|\nabla \varphi\|_{L^{p}\left(\mathbb{R}^{n}\right)}, \quad \forall \varphi \in C_{0}^{\infty}\left(\mathbb{R}^{n}\right)
$$

where $C$ is a positive constant independent of $\varphi$. A sufficient condition for (1.11) that follows from Hölder's and Sobolev's inequalities is

$$
\sigma \in L^{r}\left(\mathbb{R}^{n}\right) \quad \text { where } r=\frac{n p}{n(p-1-q)+p(1+q)}
$$

Moreover, there is an alternative charaterization of (1.11) in terms of capacities due to Maz'ya and Netrusov (see [Maz, Sec. 11.6]),

$$
\int_{0}^{\sigma\left(\mathbb{R}^{n}\right)}\left[\frac{r}{\varkappa(\sigma, r)}\right]^{\frac{1+q}{p-1-q}} d r<+\infty
$$

where $\varkappa(\sigma, r)=\inf \left\{\operatorname{cap}_{p}(E): \sigma(E) \geq r, E \subset \mathbb{R}^{n}\right.$ compact $\}$ and cap $_{p}$ is the $p$-capacity defined, for a compact set $E \subset \mathbb{R}^{n}$, by

$$
\operatorname{cap}_{p}(E)=\inf \left\{\|\nabla u\|_{L^{p}\left(\mathbb{R}^{n}\right)}^{p}: u \geq 1 \text { on } E, u \in C_{0}^{\infty}\left(\mathbb{R}^{n}\right)\right\}
$$


Thus, any one of conditions (1.7), (1.11), or (1.12), combined with (1.8), is necessary and sufficient for the existence of a positive finite energy solution to equation (1.1). The uniqueness part will be proven by first establishing the minimality of such a solution, and then using convexity of the Dirichlet integrals $\int_{\mathbb{R}^{n}}|\nabla u|^{p} d x$.

Furthermore, we are able to adjust our argument outlined above to obtain analogous results for the fractional Laplace equation (1.2) as follows.

Theorem 1.2. Let $0<q<1,0<\alpha<\frac{n}{2}$, and let $\sigma, \mu \in \mathcal{M}^{+}\left(\mathbb{R}^{n}\right)$ such that $\sigma \not \equiv 0$. Then there exists a positive finite energy solution $u \in L_{l o c}^{q}\left(\mathbb{R}^{n}, d \sigma\right) \cap \dot{H}^{\alpha}\left(\mathbb{R}^{n}\right)$ to equation (1.2) if and only if the following conditions hold:

$$
\mathbf{I}_{2 \alpha} \sigma \in L^{\frac{1+q}{1-q}}\left(\mathbb{R}^{n}, d \sigma\right)
$$

and

$$
\mu \in \dot{H}^{-\alpha}\left(\mathbb{R}^{n}\right)
$$

Moreover, if $0<\alpha \leq 1$ then such a solution is unique in $\dot{H}^{\alpha}\left(\mathbb{R}^{n}\right)$.

Here, for $0<\alpha<\frac{n}{2}$ and $\omega \in \mathcal{M}^{+}\left(\mathbb{R}^{n}\right)$, we denote by $\mathbf{I}_{2 \alpha} \omega=\mathbf{W}_{\alpha, 2} \omega$ the Riesz potential of order $2 \alpha$ (up to a normalization constant). The homogeneous Sobolev space $\dot{H}^{\alpha}\left(\mathbb{R}^{n}\right)\left(0<\alpha<\frac{n}{2}\right)$ can be defined by means of Riesz potentials,

$$
\dot{H}^{\alpha}\left(\mathbb{R}^{n}\right)=\left\{u: u=\mathbf{I}_{\alpha} f, f \in L^{2}\left(\mathbb{R}^{n}\right)\right\}
$$

equipped with norm

$$
\|u\|_{\dot{H}^{\alpha}\left(\mathbb{R}^{n}\right)}=\|f\|_{L^{2}\left(\mathbb{R}^{n}\right)} .
$$

We denote by $\dot{H}^{-\alpha}\left(\mathbb{R}^{n}\right)=\left[\dot{H}^{\alpha}\left(\mathbb{R}^{n}\right)\right]^{*}$ the space of distributions dual to $\dot{H}^{\alpha}\left(\mathbb{R}^{n}\right)$. 
Adapting the previous argument, if (1.13) holds, we first construct a positive solution $\tilde{u} \in L^{1+q}\left(\mathbb{R}^{n}, d \sigma\right)$ to the integral equation

$$
\tilde{u}=\mathbf{I}_{2 \alpha}\left(\tilde{u}^{q} d \sigma\right)+\mathbf{I}_{2 \alpha} \mu \text { in } \mathbb{R}^{n}
$$

using an iterative procedure, under the additional assumption that

$$
\mathbf{I}_{2 \alpha} \mu \in L^{1+q}\left(\mathbb{R}^{n}, d \sigma\right) .
$$

Using the nontrivial fact $(1.13) \&(1.14) \Longrightarrow(1.16)$, we deduce the existence of a solution $\tilde{u} \in L^{1+q}\left(\mathbb{R}^{n}, d \sigma\right)$ to $(1.15)$, and consequently a positive finite energy solution $u$ to equation $(1.2)$.

We observe that a sufficient condition for (1.13) that follows from Hölder's and Hardy-Littlewood-Sobolev inequalities is

$$
\sigma \in L^{r}\left(\mathbb{R}^{n}\right) \quad \text { where } r=\frac{2 n}{n(1-q)+2 \alpha(1+q)}
$$

It was shown that (1.13) is equivalent to the trace inequality

$$
\left\|\mathbf{I}_{\alpha} g\right\|_{L^{1+q}\left(\mathbb{R}^{n}, d \sigma\right)} \leq C\|g\|_{L^{2}\left(\mathbb{R}^{n}\right)}, \quad \forall g \in L^{2}\left(\mathbb{R}^{n}\right)
$$

where $C$ is a positive constant independent of $g$, see [COV2]. Thus, condition (1.13), or equivalently (1.17), equipped with condition (1.14), is necessary and sufficient for the existence of a positive finite energy solution to equation (1.2). The restriction on the value of $\alpha$ in the uniqueness result is due to availability [BF] of a certain convexity property of the Dirichlet integrals $\int_{\mathbb{R}^{n}}\left|(-\Delta)^{\frac{\alpha}{2}} u\right|^{2} d x$ in the case $\alpha \in(0,1]$.

We now consider sublinear elliptic equation (1.3) on arbitrary domains $\Omega \subseteq \mathbb{R}^{n}$ (possibly unbounded) which possess a positive Green function $G(x, y)$ associated with $\mathcal{L}$ on $\Omega \times \Omega($ see $[\mathrm{K}])$. 
An important tool in our study is the notion of the generalized energy. Suppose $u$ is a positive Green potential, i.e., $u=\mathbf{G} \omega$ for $\omega \in \mathcal{M}^{+}(\Omega)$ with $\omega \not \equiv 0$,

$$
\mathbf{G} \omega(x):=\int_{\Omega} G(x, y) d \omega(y), \quad x \in \Omega
$$

As we will show below (see Theorem 4.1), the condition (1.5) is equivalent to the generalized Green energy $\mathcal{E}_{\gamma}[\omega]$ being finite,

$$
\mathcal{E}_{\gamma}[\omega]:=\int_{\Omega}(\mathbf{G} \omega)^{\gamma} d \omega=\int_{\Omega} u^{\gamma} d \omega<+\infty
$$

which is also equivalent to $u^{\frac{\gamma+1}{2}} \in \dot{W}_{0}^{1,2}(\Omega)$. In this case, we have

$$
\int_{\Omega} u^{\gamma} d \omega=\gamma \int_{\Omega}(\mathcal{A} \nabla u \cdot \nabla u) u^{\gamma-1} d x
$$

This is well-known in the case $\gamma=1$ for the Laplacian $\mathcal{L}=-\Delta$, see [L, MZ]. Analogous integration by parts formulas with $\gamma>0$ for functions $u$ in certain Sobolev spaces with various extra restrictions on $\Omega$ and $\omega$ can be found in [MS].

Two other key ingredients in our approach are weighted norm inequalities for Green potentials of the type $\mathbf{G}: L^{r}(\Omega, d \omega) \rightarrow L^{s}(\Omega, d \omega)$ for arbitrary $\omega \in \mathcal{M}^{+}(\Omega)$, in the non-classical case $0<s<r$ and $r>1$ (Theorem 2.15), along with iterated pointwise estimates for Green potentials (Theorem 2.14) discussed below.

Employing these tools, we establish the following results.

Theorem 1.3. Let $0<q<1$ and $\gamma>0$. Suppose $G$ is a positive Green function associated with $\mathcal{L}$ in $\Omega \subseteq \mathbb{R}^{n}$. Let $\sigma, \mu \in \mathcal{M}^{+}(\Omega)$ such that $\sigma \not \equiv 0$. Then there exists a positive solution $u \in L_{l o c}^{q}(\Omega, d \sigma)$ to (1.3) which satisfies (1.5), or equivalently $u^{\frac{\gamma+1}{2}} \in \dot{W}_{0}^{1,2}(\Omega)$, if and only if the following conditions hold:

$$
\mathbf{G} \sigma \in L^{\frac{\gamma+q}{1-q}}(\Omega, d \sigma)
$$


and

$$
\mathbf{G} \mu \in L^{\gamma}(\Omega, d \mu)
$$

When $\gamma=1$, such a solution is unique in $\dot{W}_{0}^{1,2}(\Omega)$.

Corollary 1.4. Under the assumptions of Theorem 1.3, suppose that $\frac{n}{n-1}<p \leq 2$, where $n \geq 3$. If both conditions (1.20) and (1.21) hold with $\gamma:=\frac{p(n-1)-n}{n-p} \in(0,1]$, i.e.,

$$
\mathbf{G} \sigma \in L^{r}(\Omega, d \sigma) \quad \text { and } \quad \mathbf{G} \mu \in L^{s}(\Omega, d \mu)
$$

where $r:=\frac{p(n-2)}{(1-q)(n-p)}-1$ and $s:=\frac{p(n-1)-n}{n-p}$, then there exists a positive solution $u \in L_{l o c}^{q}(\Omega, d \sigma) \cap \dot{W}_{0}^{1, p}(\Omega)$ to $(1.3)$.

A sufficient condition for (1.22) is given by

$$
\sigma \in L^{r_{1}}(\Omega) \quad \text { and } \quad \mu \in L^{s_{1}}(\Omega)
$$

where $r_{1}:=\frac{n(\gamma+1)}{n(1-q)+2(\gamma+q)}$ and $s_{1}:=\frac{n(\gamma+1)}{n+2 \gamma}$ (see Proposition 5.6 below). Thus, in light of Corollary 1.4, we have the following result.

Corollary 1.5. Under the assumptions of Corollary 1.4, if

$$
\sigma \in L^{r_{2}}(\Omega) \quad \text { and } \quad \mu \in L^{s_{2}}(\Omega)
$$

where $r_{2}:=\frac{n p}{(n-p)(1-q)+2 p}$ and $s_{2}:=\frac{n p}{n+p}$, then there exists a positive solution $u \in$ $L_{l o c}^{q}(\Omega, d \sigma) \cap \dot{W}_{0}^{1, p}(\Omega)$ to $(1.3)$.

We observe that Corollary 1.5 in the case $\mu \equiv 0$ (for bounded domains $\Omega$ ) is due to Boccardo and Orsina [BO], with a different proof.

In our proof of Theorem 1.3, we start with the corresponding integral equation

$$
\tilde{u}=\mathbf{G}\left(\tilde{u}^{q} d \sigma\right)+\mathbf{G} \mu \text { in } \Omega,
$$


under some mild assumptions on the kernel $G$, which by the maximum principle are automatically satisfied by Green functions associated with elliptic operators (includ$\operatorname{ing} \mathcal{L})$ in $\Omega$.

We find a crucial relation between $\sigma$ and $\mu$, which follows from conditions (1.20) and (1.21), and yields an important two-weight condition:

$$
\mathbf{G} \mu \in L^{\gamma+q}(\Omega, d \sigma) .
$$

This supplementary fact allows us to construct a positive solution $\tilde{u} \in L^{\gamma+q}(\Omega, d \sigma) \cap$ $L^{\gamma}(\Omega, d \mu)$ to (1.25) by using an iterative procedure, under assumptions (1.20) and (1.21).

In this procedure, we employ the fact established recently in [Ver2] that condition (1.20) is equivalent to the weighted norm inequality for Green potentials,

$$
\|\mathbf{G}(f d \sigma)\|_{L^{\gamma+q}(\Omega, d \sigma)} \leq C\|f\|_{L^{\frac{\gamma+q}{q}(\Omega, d \sigma)}}, \quad \forall f \in L^{\frac{\gamma+q}{q}}(\Omega, d \sigma)
$$

where $C$ is a positive constant independent of $f$. Therefore, either (1.20) or (1.27), together with (1.21), turns out to be necessary and sufficient for the existence of a positive solution to (1.3) satisfying (1.5).

When $G$ is a positive Green function associated with $\mathcal{L}$ on $\Omega$, the integral equation (1.25) is equivalent to problem (1.3). Appealing to our characterization of the generalized Green energy, (1.5) (1.20) and (1.21) are necessary and sufficient for the existence of a positive solution $u \in L_{l o c}^{q}(\Omega, d \sigma)$ to (1.3) which satisfies (1.5).

Finally, we remark that there are some analogous results (less precise at the boundary $\partial \Omega$ ) for equation (1.1) involving the $p$-Laplace operator in domains $\Omega \subset \mathbb{R}^{n}$; see 
Remark 3.7 below. Further, in our existence results, the $p$-Laplace operator can be replaced by a more general quasilinear elliptic operator, for example the $\mathcal{A}$-Laplace operator $-\operatorname{div} \mathcal{A}(x, \nabla \cdot)$ where $\mathcal{A}(x, \xi)$ is a measurable function which satisfies standard structural assumptions ensuring that $\mathcal{A}(x, \xi) \cdot \xi \approx|\xi|^{p}$, see [CV1, HKM, MZ].

The main results presented in this dissertation are taken from joint works with Igor E. Verbitsky [SV1, SV2].

\subsection{Organization}

This work is organized as follows. In Chapter 2, we recall the necessary background facts, and collect some useful results concerning quasilinear equations and nonlinear potentials which are repeatedly referred to throughout this study. In Chapter 3, we establish explicit necessary and sufficient conditions for the existence of positive finite energy solutions to equations (1.1), (1.2) and (1.3). Uniqueness results for such solutions are also demonstrated. Chapter 4 is devoted to a characterization of the generalized Green energy. Our argument relies on the existence result for a positive finite energy solution to (1.3). In Chapter 5, we extend this result by employing such characterization to obtain necessary and sufficient conditions for the existence of a positive solutions with finite generalized energy to (1.3), and discuss its consequences. 


\section{Chapter 2}

\section{Preliminaries}

Throughout this work, let $\Omega$ be a domain (nonempty open connected set) in $\mathbb{R}^{n}$, where $n \geq 2$. Denote by $\mathcal{M}^{+}(\Omega)$ the set of all positive locally finite Borel measures on $\Omega$. Letters $c$ and $C$ stand for positive constants whose value may change at each occurrence, unless otherwise stated.

\section{$2.1 \quad$ Function spaces}

Define $C_{0}^{\infty}(\Omega)$ as the set of all smooth compactly supported functions on $\Omega$.

For $0<p<\infty$ and $\omega \in \mathcal{M}^{+}(\Omega)$, denote by $L^{p}(\Omega, d \omega)$ the space of all real-valued measurable functions $u$ on $\Omega$ such that

$$
\|u\|_{L^{p}(\Omega, d \omega)}:=\left(\int_{\Omega}|u|^{p} d \omega\right)^{\frac{1}{p}}<+\infty .
$$

The corresponding local space $L_{l o c}^{p}(\Omega, d \omega)$ consists of real-valued measurable functions $u$ on $\Omega$ such that the restriction $u_{\mid K} \in L^{p}(K, d \omega)$ for every compact set $K \subset \Omega$. When $\omega$ is ( $n$-dimensional) Lebesgue measure, $d \omega=d x$, we write $L^{p}(\Omega)$ and $L_{l o c}^{p}(\Omega)$, respectively.

For $1 \leq p<\infty$, the Sobolev space $W^{1, p}(\Omega)$ consists of all functions $u \in L^{p}(\Omega)$ 
such that $|\nabla u| \in L^{p}(\Omega)$, where

$$
\nabla u=\left(\frac{\partial u}{\partial x_{1}}, \frac{\partial u}{\partial x_{2}}, \ldots, \frac{\partial u}{\partial x_{n}}\right)
$$

is the vector of distributional partial derivatives of $u$ of order 1 . The norm on $W^{1, p}(\Omega)$ is given by

$$
\|u\|_{W^{1, p}(\Omega)}:=\|u\|_{L^{p}(\Omega)}+\|\nabla u\|_{L^{p}(\Omega)}
$$

The corresponding local space, denoted by $W_{l o c}^{1, p}(\Omega)$, is the space of all functions $u$ on $\Omega$ such that the restriction $u_{\mid D} \in W^{1, p}(D)$ for every relatively compact open set $D \subset \Omega$.

The Sobolev space $W_{0}^{1, p}(\Omega)$ is defined as the closure of $C_{0}^{\infty}(\Omega)$ in $W^{1, p}(\Omega)$. It is easy to see that $W_{0}^{1, p}\left(\mathbb{R}^{n}\right)=W^{1, p}\left(\mathbb{R}^{n}\right)$. The homogeneous version of $W_{0}^{1, p}(\Omega)$, called the homogeneous Sobolev space (or Dirichlet space), denoted by $\dot{W}_{0}^{1, p}(\Omega)$, is defined as the closure of $C_{0}^{\infty}(\Omega)$ with respect to the seminorm

$$
\|u\|_{\dot{W}_{0}^{1, p}(\Omega)}:=\|\nabla u\|_{L^{p}(\Omega)}
$$

That is, $\dot{W}_{0}^{1, p}(\Omega)$ is the set of all functions $u \in W_{l o c}^{1, p}(\Omega)$ such that $|\nabla u| \in L^{p}(\Omega)$ for which there exists a sequence $\left\{\varphi_{j}\right\}_{j=1}^{\infty} \subset C_{0}^{\infty}(\Omega)$ such that $\left\|\nabla u-\nabla \varphi_{j}\right\|_{L^{p}(\Omega)} \rightarrow 0$ as $j \rightarrow \infty$. When $1<p<n$, the dual space to $\dot{W}_{0}^{1, p}(\Omega)$, denoted by $W^{-1, p^{\prime}}(\Omega)$, is the space of distributions $\omega \in \mathcal{D}^{\prime}(\Omega)$ such that

$$
\|\omega\|_{W^{-1, p^{\prime}}(\Omega)}:=\sup \frac{|\langle\omega, u\rangle|}{\|u\|_{\dot{W}_{0}^{1, p}(\Omega)}}<+\infty
$$

where the supremum is taken over all nontrivial functions $u \in C_{0}^{\infty}(\Omega)$. Here $p^{\prime}:=\frac{p}{p-1}$ is the Hölder conjugate of $p$. For a measure $\mu \in \mathcal{M}^{+}(\Omega), \mu \in \dot{W}^{-1, p^{\prime}}(\Omega)$ if and only if there exists a positive constant $C$ such that

$$
\left|\int_{\Omega} \varphi d \mu\right| \leq C\left(\int_{\Omega}|\nabla \varphi|^{p} d x\right)^{\frac{1}{p}}, \quad \forall \varphi \in C_{0}^{\infty}(\Omega) .
$$




\subsection{Riesz potentials}

For $0<\alpha<n$, the Riesz potential $\mathbf{I}_{\alpha} f$ of a function $f \in L_{l o c}^{1}\left(\mathbb{R}^{n}\right)$ is defined by

$$
\mathbf{I}_{\alpha} f(x)=(-\Delta)^{-\frac{\alpha}{2}} f(x)=\gamma(\alpha, n) \int_{\mathbb{R}^{n}} \frac{f(y)}{|x-y|^{n-\alpha}} d y, \quad x \in \mathbb{R}^{n}
$$

where $\gamma(\alpha, n)=\frac{\Gamma\left(\frac{n-\alpha}{2}\right)}{\pi^{\frac{n}{2}} 2^{\alpha} \Gamma\left(\frac{\alpha}{2}\right)}$ is a normalization constant.

Observe that, for $f \in L^{p}\left(\mathbb{R}^{n}\right)$ and $1<p<\frac{n}{\alpha}$, the Riesz potential $\mathbf{I}_{\alpha} f$ is well-defined and finite $(\alpha, p)$-quasi everywhere (briefly, q.e.), meaning everywhere except for a set of $(\alpha, p)$-capacity zero, see $[\mathrm{AH}]$. Moreover, $\mathbf{I}_{\alpha} f$ is $(\alpha, p)$-quasicontinuous (in brief, quasicontinuous) which means that, for every $\epsilon>0$, there is an open set $E \subset \mathbb{R}^{n}$ such that $\operatorname{cap}_{\alpha, p}(E)<\epsilon$ and the restriction $\left.\mathbf{I}_{\alpha} f\right|_{E^{c}}$ is continuous on $E^{c}=\mathbb{R}^{n} \backslash E$. Here the $(\alpha, p)$-capacity of $E \subset \mathbb{R}^{n}$ is defined by

$$
\operatorname{cap}_{\alpha, p}(E):=\inf \left\{\|u\|_{L^{p}\left(\mathbb{R}^{n}\right)}^{p}: \mathbf{I}_{\alpha} u \geq 1 \text { on } E, u \geq 0 \text { a.e., } u \in L^{p}\left(\mathbb{R}^{n}\right)\right\}
$$

In a similar manner, the Riesz potential $\mathbf{I}_{\alpha} \sigma$ of order $\alpha \in(0, n)$ of a measure $\sigma \in \mathcal{M}^{+}\left(\mathbb{R}^{n}\right)$ is defined by

$$
\mathbf{I}_{\alpha} \sigma(x)=(-\Delta)^{-\frac{\alpha}{2}} \sigma(x)=(n-\alpha) \gamma(\alpha, n) \int_{0}^{\infty} \frac{\sigma(B(x, r))}{r^{n-\alpha}} \frac{d r}{r}, \quad x \in \mathbb{R}^{n}
$$

Henceforth, the normalization constant will be dropped for the sake of convenience.

For $1<p<\infty$ and $0<\alpha<\frac{n}{p}$, the fractional homogeneous Sobolev space is defined by (see [St])

$$
\dot{L}^{\alpha, p}\left(\mathbb{R}^{n}\right):=\left\{u: u=\mathbf{I}_{\alpha} f, f \in L^{p}\left(\mathbb{R}^{n}\right)\right\}
$$

equipped with norm

$$
\|u\|_{\dot{L}^{\alpha, p}\left(\mathbb{R}^{n}\right)}:=\|f\|_{L^{p}\left(\mathbb{R}^{n}\right)} .
$$


Clearly, $\dot{W}_{0}^{1, p}\left(\mathbb{R}^{n}\right)=\dot{L}^{1, p}\left(\mathbb{R}^{n}\right)$. In the case $p=2$, we use the notation

$$
\dot{L}^{\alpha, 2}\left(\mathbb{R}^{n}\right)=\dot{H}^{\alpha}\left(\mathbb{R}^{n}\right)
$$

It is well-known that when $0<\alpha<1,\|u\|_{\dot{H}^{\alpha}\left(\mathbb{R}^{n}\right)}$ is equivalent to the Gagliardo seminorm

$$
\left(\int_{\mathbb{R}^{n}} \int_{\mathbb{R}^{n}} \frac{|u(x)-u(y)|^{2}}{|x-y|^{n+2 \alpha}} d x d y\right)^{\frac{1}{2}}
$$

see, for example, [AH, Sec. 3.5].

The dual space of $\dot{L}^{\alpha, p}\left(\mathbb{R}^{n}\right)$ for $0<\alpha<\frac{n}{2}$, denoted by $\dot{L}^{-\alpha, p^{\prime}}\left(\mathbb{R}^{n}\right)$, consists of distributions $\mu \in \mathcal{D}^{\prime}\left(\mathbb{R}^{n}\right)$ such that

$$
\|\mu\|_{\dot{L}^{-\alpha, p^{\prime}}\left(\mathbb{R}^{n}\right)}=\sup \frac{|\langle\mu, u\rangle|}{\|u\|_{\dot{L}^{\alpha, p}\left(\mathbb{R}^{n}\right)}}<+\infty,
$$

where the supremum is taken over all nontrivial functions $u \in C_{0}^{\infty}\left(\mathbb{R}^{n}\right)$. In the same spirit as above when $p=2$, we use the notation $\dot{L}^{-\alpha, 2}\left(\mathbb{R}^{n}\right)=\dot{H}^{-\alpha}\left(\mathbb{R}^{n}\right)$. Thus, by duality, for a measure $\mu \in \mathcal{M}^{+}\left(\mathbb{R}^{n}\right)$ we have

$$
\mu \in \dot{H}^{-\alpha}\left(\mathbb{R}^{n}\right) \Longleftrightarrow\left\|\mathbf{I}_{\alpha} \mu\right\|_{L^{2}\left(\mathbb{R}^{n}\right)}<+\infty \Longleftrightarrow \int_{\mathbb{R}^{n}} \mathbf{I}_{2 \alpha} \mu d \mu<+\infty
$$

\subsection{Wolff potentials and energy estimates}

Let $1<p<\infty, 0<\alpha<\frac{n}{p}$ and $\sigma \in \mathcal{M}^{+}\left(\mathbb{R}^{n}\right)$. The (homogeneous) Wolff potential $\mathbf{W}_{\alpha, p} \sigma$ is defined by (see $[\mathrm{AH}],[\mathrm{KuMi}]$ )

$$
\mathbf{W}_{\alpha, p} \sigma(x)=\int_{0}^{\infty}\left[\frac{\sigma(B(x, r))}{r^{n-\alpha p}}\right]^{\frac{1}{p-1}} \frac{d r}{r}, \quad x \in \mathbb{R}^{n},
$$

where $B(x, r)=\left\{y \in \mathbb{R}^{n}:|x-y|<r\right\}$ is a ball centered at $x \in \mathbb{R}^{n}$ of radius $r>0$.

Observe that $\mathbf{W}_{\alpha, p} \sigma$ is always positive whenever $\sigma \not \equiv 0$. Moreover, either $\mathbf{W}_{\alpha, p} \sigma \equiv$ $+\infty$ or $\mathbf{W}_{\alpha, p} \sigma<+\infty$ a.e. In other words, $\mathbf{W}_{\alpha, p} \sigma<+\infty$ a.e. if and only if $\mathbf{W}_{\alpha, p} \sigma\left(x_{0}\right)<+\infty$ for some $x_{0} \in \mathbb{R}^{n}$. 
In the linear case, when $p=2, \mathbf{W}_{\alpha, 2} \sigma=\mathbf{I}_{2 \alpha} \sigma$, and in particular, $\mathbf{W}_{1,2} \sigma=\mathbf{I}_{2} \sigma$ is the Newtonian potential.

The $(\alpha, p)$-energy of $\sigma$ is given by

$$
\mathcal{E}_{\alpha, p}(\sigma):=\left\|\mathbf{I}_{\alpha} \sigma\right\|_{L^{p^{\prime}\left(\mathbb{R}^{n}\right)}}^{p^{\prime}}
$$

The fundamental Wolff's inequality, see [AH, Sec. 4.5], provides a certain estimate of the energy by means of the corresponding Wolff potential:

$$
C^{-1} \mathcal{E}_{\alpha, p}(\sigma) \leq \int_{\mathbb{R}^{n}} \mathbf{W}_{\alpha, p} \sigma d \sigma \leq C \mathcal{E}_{\alpha, p}(\sigma)
$$

where $C=C(\alpha, n, p) \geq 1$. Consequently,

$$
\mathbf{W}_{\alpha, p} \sigma \in L^{1}\left(\mathbb{R}^{n}, d \sigma\right) \Longleftrightarrow \mathcal{E}_{\alpha, p}(\sigma)<+\infty
$$

More generally, it was shown in [COV1] (see also [COV2]) that for $0 \leq q<p, p>1$,

$$
\mathbf{W}_{\alpha, p} \sigma \in L^{\frac{q(p-1)}{p-q}}\left(\mathbb{R}^{n}, d \sigma\right)
$$

is equivalent to the trace inequality

$$
\left(\int_{\mathbb{R}^{n}}\left|\mathbf{I}_{\alpha} g\right|^{q} d \sigma\right)^{\frac{1}{q}} \leq C\left(\int_{\mathbb{R}^{n}}|g|^{p} d x\right)^{\frac{1}{p}}, \quad \forall g \in L^{p}\left(\mathbb{R}^{n}\right)
$$

where $C$ is a positive constant independent of $g$. When $\alpha=k<\frac{n}{2}$ is a positive integer, (2.2) is equivalent to the generalized Sobolev inequality

$$
\left(\int_{\mathbb{R}^{n}}|g|^{q} d \sigma\right)^{\frac{1}{q}} \leq C\left(\int_{\mathbb{R}^{n}}\left|\nabla^{k} g\right|^{p} d x\right)^{\frac{1}{p}}, \quad \forall g \in C_{0}^{\infty}\left(\mathbb{R}^{n}\right)
$$

where $C$ is a positive constant independent of $g$. 


\section{$2.4 \mathcal{A}$-superharmonic functions}

Let $1<p<\infty$. Suppose $\mathcal{A}: \mathbb{R}^{n} \times \mathbb{R}^{n} \rightarrow \mathbb{R}^{n}$ be a vector-valued function which satisfies the following structural conditions:

- Carathéodory conditions:

$$
\begin{aligned}
& \text { the map } x \mapsto \mathcal{A}(x, \xi) \text { is measurable for all } \xi \in \mathbb{R}^{n} \text {; } \\
& \text { the map } \xi \mapsto \mathcal{A}(x, \xi) \text { is continuous for a.e. } x \in \mathbb{R}^{n} \text {. }
\end{aligned}
$$

- Growth conditions: there are constants $0<\alpha \leq \beta<\infty$ such that for all $\xi \in \mathbb{R}^{n}$ and for a.e. $x \in \mathbb{R}^{n}$,

$$
\mathcal{A}(x, \xi) \cdot \xi \geq \alpha|\xi|^{p} \quad \text { and } \quad|\mathcal{A}(x, \xi)| \leq \beta|\xi|^{p-1} \text {. }
$$

- Monotonicity condition: for all $\xi_{1}, \xi_{2} \in \mathbb{R}^{n}$ and for a.e. $x \in \mathbb{R}^{n}$,

$$
\left[\mathcal{A}\left(x, \xi_{1}\right)-\mathcal{A}\left(x, \xi_{2}\right)\right] \cdot\left(\xi_{1}-\xi_{2}\right)>0
$$

- Homogeneity condition: for every $\lambda \in \mathbb{R} \backslash\{0\}$, for all $\xi_{1}, \xi_{2} \in \mathbb{R}^{n}$ and for a.e. $x \in \mathbb{R}^{n}$,

$$
\mathcal{A}(x, \lambda \xi)=\lambda|\lambda|^{p-2} \mathcal{A}(x, \xi) .
$$

The $\mathcal{A}$-Laplacian is defined by

$$
\operatorname{div} \mathcal{A}(x, \nabla u)=\nabla \cdot \mathcal{A}(x, \nabla u), \quad u \in W_{l o c}^{1, p}(\Omega)
$$

in the distributional sense, i.e., for every $\varphi \in C_{0}^{\infty}(\Omega)$,

$$
\langle\operatorname{div} \mathcal{A}(x, \nabla u), \varphi\rangle=\langle\nabla \cdot \mathcal{A}(x, \nabla u), \varphi\rangle=-\int_{\Omega} \mathcal{A}(x, \nabla u) \cdot \nabla \varphi d x
$$


A prototype of the this operator is when $\mathcal{A}(x, \xi)=|\xi|^{p-2} \xi$, the so-called $p$-Laplacian defined by

$$
\Delta_{p} u=\nabla \cdot\left(|\nabla u|^{p-2} \nabla u\right), \quad u \in W_{l o c}^{1, p}(\Omega) .
$$

When $p=2$, this reduces to the classical Laplacian $\Delta$.

A function $u \in W_{l o c}^{1, p}(\Omega)$ is said to be $\mathcal{A}$-harmonic if $u$ satisfies the equation

$$
\operatorname{div} \mathcal{A}(x, \nabla u)=0 \quad \text { in } \Omega
$$

in the distributional sense, i.e.,

$$
\int_{\Omega} \mathcal{A}(x, \nabla u) \cdot \nabla \varphi d x=0, \quad \forall \varphi \in C_{0}^{\infty}(\Omega) .
$$

It was established in [HKM, Theorem 3.70] that every $\mathcal{A}$-harmonic function $u$ has a continuous representative which coincides with $u$ a.e. We always refer to an $\mathcal{A}$ harmonic function on $\Omega$ as a continuous distributional solution to (2.9) on $\Omega$, and denote by $\mathcal{H}_{\mathcal{A}}(\Omega)$ the set of all $\mathcal{A}$-harmonic functions on $\Omega$.

A function $u: \Omega \rightarrow(-\infty,+\infty]$ is $\mathcal{A}$-superharmonic if

- $u$ is lower semicontinuous in $\Omega$;

- $u \not \equiv+\infty$ in each component of $\Omega$;

- Whenever $D$ is an relatively compact open subset of $\Omega$ and $h \in C(\bar{D}) \cap \mathcal{H}_{\mathcal{A}}(D)$,

$$
h \leq u \text { on } \partial D \Longrightarrow h \leq u \text { on } D .
$$

A function $u$ on $\Omega$ is called $\mathcal{A}$-subharmonic if $-u$ is $\mathcal{A}$-superharmonic. Observe that a function $u$ is $\mathcal{A}$-harmonic if and only if it is both $\mathcal{A}$-superharmonic and $\mathcal{A}$ subharmonic, see [HKM, Lemma 7.8]. 
Every $\mathcal{A}$-superharmonic function $u$ in $\Omega$ is $p$-quasicontinuous (in brief, quasicontinuous) in $\Omega$ [HKM, Theorem 10.9], which means that for every $\epsilon>0$, there is an open set $E \subset \Omega$ such that $\operatorname{cap}_{p}(E)<\epsilon$ and the restriction $u_{\mid E^{c}}$ is continuous on $E^{c}=\Omega \backslash E$.

Here the $p$-capacity of an open set $G \subset \Omega$ is

$$
\operatorname{cap}_{p}(G):=\sup \left\{\operatorname{cap}_{p}(K): K \subset \Omega \text { compact }\right\}
$$

where the $p$-capacity of each compact set $K \subset \Omega$ is defined by

$$
\operatorname{cap}_{p}(K):=\inf \left\{\|\nabla u\|_{L^{p}(\Omega)}^{p}: u \geq 1 \text { on } K, u \in C_{0}^{\infty}(\Omega)\right\}
$$

Notice that $\operatorname{cap}_{p}(K)$ is comparable to $\operatorname{cap}_{1, p}(K)$ for each compact set $K$ in $\mathbb{R}^{n}$.

A statement is said to hold $p$-quasi-everywhere in $\Omega$ (briefly, q.e.), if it holds everywhere except for a set of $p$-capacity zero in $\Omega$.

Denote by $\mathcal{M}_{0}^{+}(\Omega)$ the class of all measures $\omega \in \mathcal{M}^{+}(\Omega)$ which are absolutely continuous with respect to the $p$-capacity, that is, $\omega(K)=0$ whenever $\operatorname{cap}_{p}(K)=0$ for every compact subset $K$ in $\Omega$. It follows by Poincaré inequality [MZ, Corollary 1.57] that Lebesgue measure is absolutely continuous with respect to the $p$-capacity.

\subsection{Notion of solutions}

Definition 2.1. Let $1<p<\infty, q>0$ and $\sigma, \mu \in \mathcal{M}^{+}(\Omega)$. A function $u$ is said to be a finite energy solution to the equation

$$
-\operatorname{div} \mathcal{A}(x, \nabla u)=\sigma u^{q}+\mu \quad \text { in } \Omega
$$

if $u \in L_{l o c}^{q}(\Omega, d \sigma) \cap \dot{W}_{0}^{1, p}(\Omega), u \geq 0 d \sigma$-a.e. and

$$
\int_{\Omega} \mathcal{A}(x, \nabla u) \cdot \nabla \varphi d x=\int_{\Omega} \varphi u^{q} d \sigma+\int_{\Omega} \varphi d \mu, \quad \varphi \in C_{0}^{\infty}(\Omega) .
$$


Let $1<p<\infty$. As we have seen in the preceding section, the $\mathcal{A}$-Laplace operator acts naturally from $W_{\text {loc }}^{1, p}(\Omega)$ locally into the dual of $W^{1, p}(\Omega)$. Therefore in order to give a meaningful definition of a solution to the equation

$$
\operatorname{div} \mathcal{A}(x, \nabla u)=\omega \quad \text { in } \Omega
$$

with general measure data $\omega \in \mathcal{M}^{+}(\Omega)$, we need to extend the notion of distributional solutions $u$ to (2.11), for $u$ not necessarily belonging to $W_{l o c}^{1, p}(\Omega)$. We will understand such solutions in the potential-theoretic sense using $\mathcal{A}$-superharmonic functions, which is equivalent to the notion of locally renormalized solutions in terms of test functions [KKT].

A positive $\mathcal{A}$-superharmonic function $u$ does not necessarily belong to $W_{l o c}^{1, p}(\Omega)$, however its truncation

$$
T_{k}(u):=\min (u, k)
$$

does for every $k \in \mathbb{N}$. The generalized (or weak) gradient of a positive $\mathcal{A}$-superharmonic function $u$ is defined by (see [HKM]):

$$
D u:=\lim _{k \rightarrow \infty} \nabla\left(T_{k}(u)\right) \quad \text { a.e. }
$$

Let $u$ be a $\mathcal{A}$-superharmonic function in $\Omega$. Then both $|D u|^{p-1}$ and $\mathcal{A}(x, D u)$, are of class $L_{l o c}^{r}(\Omega)$ for every $1 \leq r<\frac{n}{n-1}$ (Moreover, if $p>2-\frac{1}{n}$ then $D u$ coincides with the usual gradient of $u$.), see [KM1]. This allows us to define a nonnegative $\operatorname{distribution} \operatorname{div} \mathcal{A}(x, D u)$ by

$$
-\langle\operatorname{div} \mathcal{A}(x, D u), \varphi\rangle=\int_{\Omega} \mathcal{A}(x, D u) \cdot \nabla \varphi d x, \quad \varphi \in C_{0}^{\infty}(\Omega)
$$

Thus, by the Riesz Representation Theorem, there exists a unique measure $\omega[u] \in$ 
$\mathcal{M}^{+}(\Omega)$ so that $-\operatorname{div} \mathcal{A}(x, \nabla u)=\omega[u]$. The measure $\omega[u]$ is called the Riesz measure of $u$.

Definition 2.2. For $\omega \in \mathcal{M}^{+}(\Omega)$, a function $u$ is said to be a solution to the equation

$$
-\operatorname{div} \mathcal{A}(x, D u)=\omega \quad \text { in } \Omega
$$

(in the potential-theoretic sense) if $u$ is $\mathcal{A}$-superharmonic in $\Omega$ and $\omega[u]=\omega$.

Thus, for $\sigma, \mu \in \mathcal{M}^{+}(\Omega)$, a function $u$ is said to be a solution to equation (2.10)

(in the potential-theoretic sense) if $u$ is $\mathcal{A}$-superharmonic in $\Omega$ so that $u \in L_{l o c}^{q}(\Omega, d \sigma)$ and $d \omega[u]=u^{q} d \sigma+d \mu$.

A supersolution to (2.10) is a nonnegative $\mathcal{A}$-superharmonic function $u$ in $\Omega$ so that $u \in L_{l o c}^{q}(\Omega, d \sigma)$ and

$$
\int_{\Omega} \mathcal{A}(x, \nabla u) \cdot \nabla \varphi d x \geq \int_{\Omega} u^{q} \varphi d \sigma+\int_{\Omega} \varphi d \mu, \quad \varphi \in C_{0}^{\infty}(\Omega) \text { with } \varphi \geq 0 .
$$

Note that if $u \in W_{l o c}^{1, p}(\Omega)$ is a solution (or supersolution) to equation (2.10), then the generalized gradient $D u$ coincides with the regular gradient $u$. Thus $u$ is the usual distributional solution (or supersolution, repectively).

\subsection{Kernels and potentials}

Let $G: \Omega \times \Omega \rightarrow(0, \infty]$ be a positive lower semicontinuous kernel. For $\omega \in \mathcal{M}^{+}(\Omega)$, the potential of $\omega$ is defined by

$$
\mathbf{G} \omega(x):=\int_{\Omega} G(x, y) d \omega(y), \quad x \in \Omega .
$$

Observe that $\mathbf{G} \omega(x)$ is lower semi-continuous on $\Omega \times \mathcal{M}^{+}(\Omega)$ if $G(x, y)$ is lower semicontinuous on $\Omega \times \Omega$, see [Br]. 
A positive kernel $G$ on $\Omega \times \Omega$ is said to satisfy the weak maximum principle (WMP) with constant $h \geq 1$ if for any $\omega \in \mathcal{M}^{+}(\Omega)$,

$$
\sup \{\mathbf{G} \omega(x): x \in \operatorname{supp}(\omega)\} \leq 1 \Longrightarrow \sup \{\mathbf{G} \omega(x): x \in \Omega\} \leq h .
$$

Here we use the notation $\operatorname{supp}(\omega)$ for the support of $\omega$.

When $h=1$ in (2.12), the kernel $G$ is said to satisfy the strong maximum principle. It holds for Green functions associated with the classical Laplacian $-\Delta$, or more generally the linear uniformly elliptic operator in divergence form $\mathcal{L}$, as well as the fractional Laplacian $(-\Delta)^{\alpha}$ in the case $0<\alpha \leq 1$, in every domain $\Omega \subset \mathbb{R}^{n}$ which possesses a Green function.

The WMP holds for Riesz kernels on $\mathbb{R}^{n}$ associated with $(-\Delta)^{\alpha}$ in the full range $0<\alpha<\frac{n}{2}$, and more generally for all radially nonincreasing kernels on $\mathbb{R}^{n}$, see $[\mathrm{AH}]$.

We say that a positive kernel $G$ on $\Omega \times \Omega$ is quasi-symmetric if there exists a constant $a \geq 1$ such that

$$
a^{-1} G(y, x) \leq G(x, y) \leq a G(y, x), \quad x, y \in \Omega
$$

When $a=1$ in (2.13), the kernel $G$ is said to be symmetric. There are many kernels associated with elliptic operators that are quasi-symmetric and satisfy the WMP, see $[\mathrm{An}]$.

We summarize that the Green function $G$ associated with $\mathcal{L}$ on $\Omega$ is a positive lower semicontinuous symmetric kernel, which satisfies the strong maximum principle [K, LSW]. Further, for $\omega \in \mathcal{M}^{+}(\Omega)$, Green potential $\mathbf{G} \omega$ is either $\mathcal{A}$-superharmonic or identically $+\infty$, in each component of $\Omega$, see [GH]. 


\section{7 $\quad$ Some known results}

We shall need the following weak continuity result established in [TW].

Theorem 2.3 ([TW]). Suppose $\left\{u_{j}\right\}_{j=1}^{\infty}$ is a sequence of positive $\mathcal{A}$-superharmonic functions in $\Omega$ such that $u_{j} \rightarrow u$ a.e. as $j \rightarrow \infty$, where $u$ is an $\mathcal{A}$-superharmonic function in $\Omega$. Then $\omega\left[u_{j}\right]$ converges weakly to $\omega[u]$, that is,

$$
\lim _{j \rightarrow \infty} \int_{\Omega} \varphi d \omega\left[u_{j}\right]=\int_{\Omega} \varphi d \omega[u], \quad \forall \varphi \in C_{0}^{\infty}(\Omega)
$$

The next theorem provides lower bounds for supersolutions.

Theorem 2.4 ([CV1]). Let $1<p<n, 0<q<p-1$ and $\sigma \in \mathcal{M}^{+}\left(\mathbb{R}^{n}\right)$. Suppose $u$ is a nontrivial supersolution to homogeneous equation

$$
-\operatorname{div} \mathcal{A}(x, \nabla u)=\sigma u^{q} \quad \text { in } \mathbb{R}^{n} .
$$

Then $u$ satisfies the inequality

$$
u \geq c\left(\mathbf{W}_{1, p} \sigma\right)^{\frac{p-1}{p-1-q}}
$$

where $c=c(n, p, q)>0$.

Theorem 2.5 ([CV2]). Let $1<p<n, 0<q<p-1,0<\alpha<\frac{n}{p}$ and $\sigma \in \mathcal{M}^{+}\left(\mathbb{R}^{n}\right)$.

Suppose $u \in L_{l o c}^{q}\left(\mathbb{R}^{n}, d \sigma\right)$ satisfying

$$
u \geq \mathbf{W}_{\alpha, p}\left(u^{q} d \sigma\right) \quad d \sigma-a . e .
$$

Then, $u$ satisfies the inequality

$$
u \geq c\left(\mathbf{W}_{\alpha, p} \sigma\right)^{\frac{p-1}{p-1-q}} \quad d \sigma-a . e .
$$

where $c=c(\alpha, n, p, q)>0$. 
The following important result, [KM2], is concerned with pointwise estimate of nonnegative $p$-superharmonic functions in terms of Wolff's potential.

Theorem 2.6 ([KM2]). Let $1<p<n$ and $\omega \in \mathcal{M}^{+}\left(\mathbb{R}^{n}\right)$ Suppose $u$ is a $\mathcal{A}$ superharmonic function in $\mathbb{R}^{n}$ satisfying

$$
\left\{\begin{array}{l}
-\operatorname{div} \mathcal{A}(x, \nabla u)=\omega \quad \text { in } \mathbb{R}^{n} \\
\liminf _{|x| \rightarrow \infty} u(x)=0
\end{array}\right.
$$

Then

$$
K^{-1} \mathbf{W}_{1, p} \omega \leq u \leq K \mathbf{W}_{1, p} \omega
$$

where $K=K(n, p) \geq 1$.

The next three lemmas are discussed in [CV1], which will be used in our arguments occasionally.

Lemma 2.7 ([CV1]). Let $1<p<n, 0<q<p-1$ and $\sigma \in \mathcal{M}^{+}\left(\mathbb{R}^{n}\right)$. Suppose there exists a nontrivial supersolution $u \in L_{l o c}^{q}\left(\mathbb{R}^{n}, d \sigma\right) \cap \dot{W}_{0}^{1, p}\left(\mathbb{R}^{n}\right)$ to equation (1.6) Then

$$
-\Delta_{p} u \in W^{-1, p^{\prime}}\left(\mathbb{R}^{n}\right) \cap \mathcal{M}^{+}\left(\mathbb{R}^{n}\right) \quad \text { and } \quad u \in L^{1+q}\left(\mathbb{R}^{n}, d \sigma\right),
$$

for a quasicontinuous representative of u. Consequently, (1.7) holds.

Lemma 2.8 ([CV1]). Suppose $u \in L^{1+q}\left(\mathbb{R}^{n}, d \sigma\right)$ is a nontrivial supersolution to the integral equation

$$
u=\mathbf{W}_{1, p}\left(u^{q} d \sigma\right) \quad d \sigma-a . e .
$$

Then

$$
u^{q} d \sigma \in W^{-1, p^{\prime}}\left(\mathbb{R}^{n}\right) \cap \mathcal{M}^{+}\left(\mathbb{R}^{n}\right)
$$


Lemma $2.9([\mathrm{CV} 1])$. Let $\mu, \omega \in W^{-1, p}\left(\mathbb{R}^{n}\right) \cap \mathcal{M}^{+}\left(\mathbb{R}^{n}\right)$. Suppose $u, v \in \dot{W}_{0}^{1, p}\left(\mathbb{R}^{n}\right)$ are solutions to the equations

$$
-\Delta_{p} u=\mu \quad \text { in } \mathbb{R}^{n} \quad \text { and } \quad-\Delta_{p} v=\omega \quad \text { in } \mathbb{R}^{n}
$$

respectively. If $\mu \leq \omega$, then $u \leq v$ q.e.

The following theorem is due to Brezis and Browder [BrB] (cf. [MZ, Theorem $2.39])$.

Theorem 2.10. Let $1<p<n$ and $\mu \in W^{-1, p^{\prime}}(\Omega) \cap \mathcal{M}^{+}(\Omega)$. Then for any $u \in$ $\dot{W}_{0}^{1, p}(\Omega)$ we have $u \in L^{1}(\Omega, d \mu)$ and

$$
\langle\mu, u\rangle=\int_{\Omega} u d \mu
$$

for a quasicontinuous representative of $u$.

We shall use the following facts, which are discussed in [MZ, Secs. 2.1-2.2].

Remark 2.11. Let $1<p<n$ and $\omega \in W^{-1, p^{\prime}}\left(\mathbb{R}^{n}\right) \cap \mathcal{M}^{+}\left(\mathbb{R}^{n}\right)$. There exists a unique $p$-superharmonic solution $u \in \dot{W}_{0}^{1, p}\left(\mathbb{R}^{n}\right)$ to the equation

$$
-\Delta_{p} u=\omega \quad \text { in } \mathbb{R}^{n}
$$

in the distributional sense. Moreover, $u \in L^{1}\left(\mathbb{R}^{n}, d \omega\right)$ and

$$
\langle\omega, u\rangle=\int_{\mathbb{R}^{n}} u d \omega=\|u\|_{\dot{W}_{0}^{1, p}\left(\mathbb{R}^{n}\right)}^{p}=\|\omega\|_{W^{-1, p^{\prime}}\left(\mathbb{R}^{n}\right)}^{p^{\prime}}=\left\|I_{1} \omega\right\|_{L^{p^{\prime}\left(\mathbb{R}^{n}\right)}}^{p^{\prime}}=\mathcal{E}_{1, p}(\omega)
$$

for a quasicontinuous representative of $u$.

We will need the next lemma which shows that if there exists a nontrivial supersolution $u \in L_{l o c}^{q}\left(\mathbb{R}^{n}, d \sigma\right)$ to the integral equation

$$
u=\mathbf{W}_{\alpha, p}\left(u^{q} d \sigma\right) \quad d \sigma-a . e .
$$


then $\sigma$ must be absolutely continuous with respect to cap ${ }_{\alpha, p}$.

Lemma 2.12 ([CV2]). Let $1<p<\infty, 0<\alpha<\frac{n}{p}$ and $0<q<p-1$ and $\sigma \in \mathcal{M}^{+}\left(\mathbb{R}^{n}\right)$. Suppose there exists a nontrivial supersolution $u \in L_{\text {loc }}^{q}\left(\mathbb{R}^{n}, d \sigma\right)$ to (2.15). Then there exists a positive constant c such that

$$
\sigma(E) \leq c\left[\operatorname{cap}_{\alpha, p}(E)\right]^{\frac{q}{p-1}}\left(\int_{E} u^{q} d \sigma\right)^{\frac{p-1-q}{p-1}}
$$

for all compact sets $E \subset \mathbb{R}^{n}$.

Consequently, if (1.6) has a nontrivial p-superharmonic supersolution then $\sigma$ is absolutely continuous with respect to cap $_{p}$.

The following theorem provides pointwise estimates for supersolutions to sublinear elliptic equations, see [GV, Theorem 1.3].

Theorem $2.13([\mathrm{GV}])$. Let $0<q<1, \omega \in \mathcal{M}^{+}(\Omega)$, and let $G$ be a positive lower semicontinuous kernel on $\Omega \times \Omega$, which satisfies the WMP with constant $h \geq 1$. If $u \in L_{\text {loc }}^{q}(\Omega, d \omega)$ is a positive solution to the integral inequality

$$
u \geq \mathbf{G}\left(u^{q} d \omega\right) \quad \text { in } \Omega
$$

then

$$
u(x) \geq(1-q)^{\frac{1}{1-q}} h^{\frac{-q}{1-q}}[\mathbf{G} \omega(x)]^{\frac{1}{1-q}}, \quad \forall x \in \Omega .
$$

The following pair of iterated pointwise inequalities plays an important role in this paper (see [GV, Lemma 2.5]).

Theorem $2.14([\mathrm{GV}])$. Let $\omega \in \mathcal{M}^{+}(\Omega)$, and let $G$ be a positive lower semicontinuous kernel on $\Omega \times \Omega$, which satisfies the WMP with constant $h \geq 1$. Then the following estimates hold: 
(i) If $s \geq 1$, then

$$
(\mathbf{G} \omega)^{s}(x) \leq s h^{s-1} \mathbf{G}\left((\mathbf{G} \omega)^{s-1} d \omega\right)(x), \quad \forall x \in \Omega
$$

(ii) If $0<s \leq 1$, then

$$
(\mathbf{G} \omega)^{s}(x) \geq \operatorname{sh}^{s-1} \mathbf{G}\left((\mathbf{G} \omega)^{s-1} d \omega\right)(x), \quad \forall x \in \Omega .
$$

Our argument also relies on the following result established in [Ver2, Theorem 1.1], which explicitly characterizes $(p, r)$-weighted norm inequalities

$$
\|\mathbf{G}(f d \omega)\|_{L^{r}(\Omega, d \omega)} \leq C\|f\|_{L^{p}(\Omega, d \omega)}, \quad \forall f \in L^{p}(\Omega, d \omega)
$$

where $C$ is a positive constant independent of $f$, in the case $0<r<p$ and $1<p<\infty$, for arbitrary $\omega \in \mathcal{M}^{+}(\Omega)$, under certain assumptions on the kernel $G$.

Theorem 2.15 ([Ver2]). Let $\omega \in \mathcal{M}^{+}(\Omega)$ with $\omega \not \equiv 0$, and let $G$ be a positive quasisymmetric lower semicontinuous kernel on $\Omega \times \Omega$, which satisfies the WMP.

(i) If $1<p<\infty$ and $0<r<p$, then the $(p, r)$-weighted norm inequality (2.20) holds if and only if

$$
\mathbf{G} \omega \in L^{\frac{p r}{p-r}}(\Omega, d \omega)
$$

(ii) If $0<q<1$ and $q<r<\infty$, then there exists a positive solution $u \in L^{r}(\Omega, d \omega)$ to the integral inequality (2.16) if and only if the weighted norm inequality (2.20) holds with $p=\frac{r}{q}$, that is,

$$
\|\mathbf{G}(f d \omega)\|_{L^{r}(\Omega, d \omega)} \leq C\|f\|_{L^{\frac{r}{q}}(\Omega, d \omega)}, \quad \forall f \in L^{\frac{r}{q}}(\Omega, d \omega)
$$

where $C$ is a positive constant independent of $f$; or equivalently,

$$
\mathbf{G} \omega \in L^{\frac{r}{1-q}}(\Omega, d \omega)
$$


The following inequalities are often used in the theory of Schrödinger operators and potential theory. They can be found, for example, in [HKM, Theorem 7.48] and [JMV, Proposition 1.5], respectively.

Theorem 2.16 ([HKM, JMV]). Let $\omega \in \mathcal{M}^{+}(\Omega)$ with $\omega \not \equiv 0$, and let $G$ be a positive Green function associated with $\mathcal{L}$ on $\Omega$. Suppose $u:=\mathbf{G} \omega$ so that $u \not \equiv+\infty$. Then there exists a positive constant $C$ which depends only on the ellipticity constants $m, M$ such that

$$
\int_{\Omega}|\varphi|^{2} \frac{|\nabla u|^{2}}{u^{2}} d x \leq C \int_{\Omega}|\nabla \varphi|^{2} d x
$$

and

$$
\int_{\Omega}|\varphi|^{2} \frac{d \omega}{u} \leq C \int_{\Omega}|\nabla \varphi|^{2} d x
$$

for all (quasicontinuous representatives of) $\varphi \in \dot{W}_{0}^{1,2}(\Omega)$.

Proof. With out loss of generality, we assume $\mathcal{L}=-\Delta$ due to uniform ellipticity condition (1.4). Observe that since $\sigma \not \equiv 0$ and $u \not \equiv+\infty$ in $\Omega, u$ is a positive superharmonic function in $\Omega$, and thus $u$ is quasicontinuous in $\Omega$. In particular, $u$ is finite q.e. in $\Omega$ (see [MZ, Theorem 2.68]). Further, the integrals on the left-hand side of (2.24) and (2.25) are well-defined because $\log u \in W_{l o c}^{1,2}(\Omega)$ (see [HKM, Theorem 7.48]) and $\frac{d \sigma}{u} \in \mathcal{M}^{+}(\Omega)$ since $u$ is bounded below by a positive constant on each compact subset of $\Omega$.

It suffices to establish the inequalities (2.24) and (2.25) for all test functions $\varphi \in$ $C_{0}^{\infty}(\Omega)$. In fact, if $\nu \in \mathcal{M}^{+}(\Omega)$ satisfying

$$
\int_{\Omega}|\varphi|^{2} d \nu \leq C \int_{\Omega}|\nabla \varphi|^{2} d x, \quad \forall \varphi \in C_{0}^{\infty}(\Omega)
$$

where $c$ is a positive constant independent of $\varphi$. Then, in view of the definition of the capacity, we have $\nu(K) \leq C \operatorname{cap}(K)$ for every compact set $K \subset \Omega$. In particular, 
$\nu \in \mathcal{M}_{0}^{+}(\Omega)$. Taking this into account (with measures $d \nu:=\nabla \log u d x$ and $d \nu:=$ $\frac{d \sigma}{u}$, respectively, in $\left.(2.26)\right)$, together with a standard density argument, we see that both inequalities (2.24) and (2.25) are valid for quasicontinuous representatives of $\varphi \in \dot{W}_{0}^{1,2}(\Omega)$

Given $\varphi \in C_{0}^{\infty}(\Omega)$. Without loss of generality, we may assume $\varphi \geq 0$. For fixed $k \in \mathbb{N}$, set $u_{k}=\min (u, k)$ which is a nonnegative bounded superharmonic function of the class $W_{l o c}^{1,2}(\Omega)$, and denote by $\sigma_{k}$ the Riesz measure of $u_{k}$. As in [MZ, Lemma 2.64], pick a nonnegative $C_{0}^{\infty}$-mollifier $\phi$ such that

$$
u_{k}^{(j)}:=u_{k} * \phi_{j}, \quad j \geq k
$$

is a nonnegative smooth superharmonic function and $u_{k}^{(j)} \leq u_{k}$ in $\Omega_{j}$, where $\phi_{j}=$ $j^{n} \phi(j x)$ and $\Omega_{j}=\left\{x \in \Omega: \operatorname{dist}(x, \partial \Omega)>\frac{1}{j}\right\}$. Denote by $\sigma_{k}^{(j)}$ the Riesz measure of $u_{k}^{(j)}$. For $j$ sufficiently large so that $\operatorname{supp} \varphi \subset \Omega_{j}$. Using integration by parts, together with Schwarz's inequality, we deduce that

$$
\begin{aligned}
\int_{\Omega} \varphi^{2} \frac{d \sigma_{k}^{(j)}}{u_{k}^{(j)}}+\int_{\Omega} \varphi^{2} \frac{\left|\nabla u_{k}^{(j)}\right|^{2}}{\left(u_{k}^{(j)}\right)^{2}} d x & =-\int_{\Omega} \varphi^{2} \nabla \cdot\left(\frac{\nabla u_{k}^{(j)}}{u_{k}^{(j)}}\right) d x \\
& =\int_{\Omega} \nabla\left(\varphi^{2}\right) \cdot \frac{\nabla u_{k}^{(j)}}{u_{k}^{(j)}} d x \\
& =2 \int_{\Omega} \nabla \varphi \cdot\left(\varphi \frac{\nabla u_{k}^{(j)}}{u_{k}^{(j)}}\right) d x \\
& \leq 2\left(\int_{\Omega}|\nabla \varphi|^{2} d x\right)^{\frac{1}{2}}\left(\int_{\Omega} \frac{\varphi^{2}}{\left|\nabla u_{k}^{(j)}\right|^{2}} \frac{\left(u_{k}^{(j)}\right)^{2}}{} d x\right)^{\frac{1}{2}} .
\end{aligned}
$$

On the one hand (2.27) yields, in particular, that

$$
\int_{\Omega} \varphi^{2} \frac{\left|\nabla u_{k}^{(j)}\right|^{2}}{\left(u_{k}^{(j)}\right)^{2}} d x \leq 2\left(\int_{\Omega}|\nabla \varphi|^{2} d x\right)^{\frac{1}{2}}\left(\int_{\Omega} \varphi^{2} \frac{\left|\nabla u_{k}^{(j)}\right|^{2}}{\left(u_{k}^{(j)}\right)^{2}} d x\right)^{\frac{1}{2}}
$$

that is,

$$
\int_{\Omega} \varphi^{2} \frac{\left|\nabla u_{k}^{(j)}\right|^{2}}{\left(u_{k}^{(j)}\right)^{2}} d x \leq 4 \int_{\Omega}|\nabla \varphi|^{2} d x
$$


Letting $j \rightarrow \infty$ in $(2.29)$, we get

$$
\int_{\Omega} \varphi^{2} \frac{\left|\nabla u_{k}\right|^{2}}{u_{k}^{2}} d x \leq 4 \int_{\Omega}|\nabla \varphi|^{2} d x
$$

by means of mollification and Fatou's lemma. The inequality (2.24) follows by letting $k \rightarrow \infty$ in (2.30) using the monotone convergence theorem.

On the other hand, it is clear that

$$
2\left(\int_{\Omega} \varphi^{2} \frac{d \sigma_{k}^{(j)}}{u_{k}^{(j)}}\right)^{\frac{1}{2}}\left(\int_{\Omega} \varphi^{2} \frac{\left|\nabla u_{k}^{(j)}\right|^{2}}{\left(u_{k}^{(j)}\right)^{2}} d x\right)^{\frac{1}{2}} \leq \int_{\Omega} \varphi^{2} \frac{d \sigma_{k}^{(j)}}{u_{k}^{(j)}}+\int_{\Omega} \varphi^{2} \frac{\left|\nabla u_{k}^{(j)}\right|^{2}}{\left(u_{k}^{(j)}\right)^{2}} d x .
$$

Hence, by (2.27) and (2.31), we have

$$
\int_{\Omega} \varphi^{2} \frac{d \sigma_{k}^{(j)}}{u_{k}^{(j)}} \leq \int_{\Omega}|\nabla \varphi|^{2} d x
$$

By the construction, $u_{k}^{(j)} \leq u$ in $\operatorname{supp} \varphi$. Therefore,

$$
\int_{\Omega} \varphi^{2} \frac{d \sigma_{k}^{(j)}}{u} \leq \int_{\Omega}|\nabla \varphi|^{2} d x
$$

Since $\sigma_{k}^{(j)} \rightarrow \sigma_{k}$ in $\mathcal{M}^{+}(\Omega)$, as $j \rightarrow \infty$ [L, Lemma 0.2], and $\sigma_{k} \rightarrow \sigma$ weakly, as $k \rightarrow \infty$, then, by passing to the limit first $j \rightarrow \infty$ and then $k \rightarrow \infty$ in (2.33), the inequality (2.25) follows by using [L, Lemma 0.1] and Theorem 2.3. This completes the proof of the lemma.

Remark 2.17. When $\Omega=\mathbb{R}^{n}$ and $\mathcal{L}=-\Delta$, the constants 4 and 1 , respectively, in (2.24) and (2.25), are sharp [MV, Remark 4]. Further, there is a $L^{p}$-analogue of (2.25) established in [Ver1, Theorem 1.11], i.e., with nonlinear Wolff's potential in place of $u=\mathbf{G} \sigma$, and with a different constant. 


\section{Chapter 3}

\section{Finite energy solutions}

\subsection{Existence result for equation (1.1)}

In this section, we establish necessary and sufficient conditions for the existence of a positive finite energy solution to equation (1.1). Minimality of such a solution is demonstrated as well. In the case $p \geq n$, it follows immediately from the result in [CV1] that there is only a trivial supersolution to (1.1). Henceforth, we assume $1<p<n$.

Our first theorem is stated in the general framework of nonlinear integral equations involving Wolff potentials,

$$
u=\mathbf{W}_{\alpha, p}\left(u^{q} d \sigma\right)+\mathbf{W}_{\alpha, p} \mu \quad \text { in } \mathbb{R}^{n}
$$

where $1<p<n, 0<q<p-1,0<\alpha<\frac{n}{p}$ and $\sigma, \mu \in \mathcal{M}^{+}\left(\mathbb{R}^{n}\right)$ so that $\sigma \not \equiv 0$. This theorem will be used to construct positive finite energy solutions to both equations (1.1) and (1.2) in the cases $\alpha=1$ and $p=2$, respectively.

Theorem 3.1. Let $1<p<n, 0<q<p-1,0<\alpha<\frac{n}{p}$ and $\sigma, \mu \in \mathcal{M}^{+}\left(\mathbb{R}^{n}\right)$ so that $\sigma \not \equiv 0$. Suppose that the following conditions hold:

$$
\mathbf{W}_{\alpha, p} \sigma \in L^{\frac{(1+q)(p-1)}{p-1-q}}\left(\mathbb{R}^{n}, d \sigma\right)
$$


and

$$
\mathbf{W}_{\alpha, p} \mu \in L^{1+q}\left(\mathbb{R}^{n}, d \sigma\right)
$$

Then there exists a positive solution $u \in L^{1+q}\left(\mathbb{R}^{n}, d \sigma\right)$ to integral equation (3.1).

The following result will be used in the proof of Theorem 3.1 (see [CV1, Lemma 3.3], or [COV2] for more generality).

Lemma $3.2([\mathrm{CV} 1])$. Let $1<p<\infty, 0<q<p-1,0<\alpha<\frac{n}{p}$ and $\sigma \in \mathcal{M}^{+}\left(\mathbb{R}^{n}\right)$. Suppose (3.2) holds. Then the nonlinear integral operator $T$ defined by

$$
T(g):=\left(\mathbf{W}_{\alpha, p}(|g| d \sigma)\right)^{p-1}, \quad g \in L^{\frac{1+q}{q}}\left(\mathbb{R}^{n}, d \sigma\right)
$$

is bounded from $L^{\frac{1+q}{q}}\left(\mathbb{R}^{n}, d \sigma\right)$ to $L^{\frac{1+q}{p-1}}\left(\mathbb{R}^{n}, d \sigma\right)$.

Proof of Theorem 3.1. Without loss of generality we may assume that $g \geq 0, g \in$ $L^{\frac{1+q}{q}}\left(\mathbb{R}^{n}, d \sigma\right)$. Since $(3.2)$ holds, then by Lemma 3.2 , there exists a positive constant $c$ such that

$$
\left(\int_{\mathbb{R}^{n}}\left|\mathbf{W}_{\alpha, p}(g d \sigma)\right|^{1+q} d \sigma\right)^{\frac{1}{1+q}} \leq c\left(\int_{\mathbb{R}^{n}}|g|^{\frac{1+q}{q}} d \sigma\right)^{\frac{q}{(1+q)(p-1)}},
$$

where $c$ is a positive constant that does not depend on $g \in L^{\frac{1+q}{q}}\left(\mathbb{R}^{n}, d \sigma\right)$.

In the homogeneous case $\mu \equiv 0$, we can construct a monotone increasing sequence of positive functions $\left\{u_{j}\right\}_{j=0}^{\infty} \subset L^{1+q}\left(\mathbb{R}^{n}, d \sigma\right)$ by setting

$$
u_{0}:=\kappa\left(\mathbf{W}_{\alpha, p} \sigma\right)^{\frac{1}{1-q}} \quad \text { and } \quad u_{j+1}:=\mathbf{W}_{\alpha, p}\left(u_{j}^{q} d \sigma\right), \quad \text { for } j \in \mathbb{N}_{0}
$$

where $\kappa>0$ is chosen to be sufficiently small. Then its pointwise limit $u:=\lim _{j \rightarrow \infty} u_{j}$ is a positive solution of the class $L^{1+q}\left(\mathbb{R}^{n}, d \sigma\right)$ to $(3.1)$, by the monotone convergence theorem (cf. [CV1, Theorem 3.5]). 
In the inhomogeneous case $\mu \not \equiv 0$, we set

$$
u_{0}:=\mathbf{W}_{\alpha, p} \mu \quad \text { and } \quad u_{j+1}:=\mathbf{W}_{\alpha, p}\left(u_{j}^{q} d \sigma\right)+\mathbf{W}_{\alpha, p} \mu, \quad j \in \mathbb{N}_{0} .
$$

Observe that $u_{0}>0$ since $\mu \not \equiv 0$, and

$$
u_{1}=\mathbf{W}_{\alpha, p}\left(u_{0}^{q} d \sigma\right)+u_{0} \geq u_{0} .
$$

Suppose $u_{0} \leq u_{1} \leq \ldots \leq u_{j}$ for some $j \in \mathbb{N}$. Then

$$
u_{j+1}=\mathbf{W}_{\alpha, p}\left(u_{j}^{q} d \sigma\right)+\mathbf{W}_{\alpha, p} \mu \geq \mathbf{W}_{\alpha, p}\left(u_{j-1}^{q} d \sigma\right)+\mathbf{W}_{\alpha, p} \mu=u_{j}
$$

Hence, by induction, $\left\{u_{j}\right\}_{j=0}^{\infty}$ is a nondecreasing sequence of positive functions. Moreover, each $u_{j} \in L^{1+q}\left(\mathbb{R}^{n}, d \sigma\right)$. To see this, notice that by assumption (3.3), we have

$$
u_{0}=\mathbf{W}_{\alpha, p} \mu \in L^{1+q}\left(\mathbb{R}^{n}, d \sigma\right) .
$$

Suppose $u_{0}, \ldots, u_{j} \in L^{1+q}\left(\mathbb{R}^{n}, d \sigma\right)$ for some $j \in \mathbb{N}$. By Minkowski's inequality,

$$
\begin{aligned}
\left\|u_{j+1}\right\|_{L^{1+q}\left(\mathbb{R}^{n}, d \sigma\right)} & =\left\|\mathbf{W}_{\alpha, p}\left(u_{j}^{q} d \sigma\right)+\mathbf{W}_{\alpha, p} \mu\right\|_{L^{1+q}\left(\mathbb{R}^{n}, d \sigma\right)} \\
& \leq\left\|\mathbf{W}_{\alpha, p}\left(u_{j}^{q} d \sigma\right)\right\|_{L^{1+q}\left(\mathbb{R}^{n}, d \sigma\right)}+\left\|\mathbf{W}_{\alpha, p} \mu\right\|_{L^{1+q}\left(\mathbb{R}^{n}, d \sigma\right)} .
\end{aligned}
$$

The first term on the right-hand side of (3.5) is estimated by applying (3.4) with $g:=u_{j}^{q} \in L^{\frac{1+q}{q}}\left(\mathbb{R}^{n}, d \sigma\right)$. In fact

$$
\begin{aligned}
\left\|\mathbf{W}_{\alpha, p}\left(u_{j}^{q} d \sigma\right)\right\|_{L^{1+q}\left(\mathbb{R}^{n}, d \sigma\right)} & \leq c\left(\int_{\mathbb{R}^{n}} u_{j}^{1+q} d \sigma\right)^{\frac{q}{(1+q)(p-1)}} \\
& \leq c\left(\int_{\mathbb{R}^{n}} u_{j+1}^{1+q} d \sigma\right)^{\frac{q}{(1+q)(p-1)}} \\
& =c\left\|u_{j+1}\right\|_{L^{1+q}\left(\mathbb{R}^{n}, d \sigma\right)^{\circ}}^{\frac{q}{p-1}}
\end{aligned}
$$

Combining (3.5) and (3.6), we arrive at

$$
\left\|u_{j+1}\right\|_{L^{1+q}\left(\mathbb{R}^{n}, d \sigma\right)} \leq c\left\|u_{j+1}\right\|_{L^{1+q}\left(\mathbb{R}^{n}, d \sigma\right)}^{\frac{q}{p-1}}+\left\|\mathbf{W}_{\alpha, p} \mu\right\|_{L^{1+q}\left(\mathbb{R}^{n}, d \sigma\right)} .
$$


We estimate the first term on the right-hand side of (3.7) using Young's inequality,

$$
c\left\|u_{j+1}\right\|_{L^{1+q}\left(\mathbb{R}^{n}, d \sigma\right)}^{\frac{q}{p-1}} \leq \frac{q}{p-1}\left\|u_{j+1}\right\|_{L^{1+q}\left(\mathbb{R}^{n}, d \sigma\right)}+\frac{p-1-q}{p-1} c^{\frac{p-1}{p-1-q}}
$$

Hence, by (3.7) and (3.8), we obtain

$$
\left\|u_{j+1}\right\|_{L^{1+q}\left(\mathbb{R}^{n}, d \sigma\right)} \leq c^{\frac{p-1}{p-1-q}}+\frac{p-1}{p-1-q}\left\|\mathbf{W}_{\alpha, p} \mu\right\|_{L^{1+q}\left(\mathbb{R}^{n}, d \sigma\right)}<+\infty
$$

By induction, we have shown that each $u_{j} \in L^{1+q}\left(\mathbb{R}^{n}, d \sigma\right)$. Finally, applying the Monotone Convergence Theorem to the sequence $\left\{u_{j}\right\}_{j=0}^{\infty}$, we see that the pointwise limit

$$
u:=\lim _{j \rightarrow \infty} u_{j}
$$

exists so that $u>0, u \in L^{1+q}\left(\mathbb{R}^{n}, d \sigma\right)$ and satisfies (3.1).

Remark 3.3. The converse to Theorem 3.1 is also true in a more general sense. In fact, if $u \in L^{1+q}\left(\mathbb{R}^{n}, d \sigma\right), u>0 d \sigma$-a.e., satisfies the equation

$$
u=\mathbf{W}_{\alpha, p}\left(u^{q} d \sigma\right)+\mathbf{W}_{\alpha, p} \mu \quad d \sigma-a . e .,
$$

then obviously $u \in L_{l o c}^{q}\left(\mathbb{R}^{n}, d \sigma\right)$ by Hölder's inequality, and

$$
u \geq \mathbf{W}_{\alpha, p}\left(u^{q} d \sigma\right) \quad d \sigma-a . e .
$$

Applying Theorem 2.5, we obtain a lower pointwise estimate of $u$,

$$
u \geq c\left(\mathbf{W}_{\alpha, p} \sigma\right)^{\frac{p-1}{p-1-q}} \quad d \sigma-a . e .
$$

where $c=c(\alpha, n, p, q)>0$. This implies that (3.2) holds since $u \in L^{1+q}\left(\mathbb{R}^{n}, d \sigma\right)$. Similary, (3.3) holds because $u \in L^{1+q}\left(\mathbb{R}^{n}, d \sigma\right)$ and

$$
u \geq \mathbf{W}_{\alpha, p} \mu \quad d \sigma-a . e .
$$


The next lemma is our main observation in this section. It gives us a relation between conditions (3.2), (3.3) and

$$
\mathcal{E}_{\alpha, p}(\mu)<+\infty \Longleftrightarrow \int_{\mathbb{R}^{n}}\left|\mathbf{I}_{\alpha} \mu\right|^{p^{\prime}} d x<+\infty \Longleftrightarrow \mu \in \dot{L}^{-\alpha, p^{\prime}}\left(\mathbb{R}^{n}\right)
$$

In particular case $\alpha=1$, this yields a relation between conditions (1.7), (1.8) and (1.9), respectively.

Lemma 3.4. Let $1<p<n, 0<q<p-1,0<\alpha<\frac{n}{p}$ and $\sigma, \mu \in \mathcal{M}^{+}\left(\mathbb{R}^{n}\right)$. Then conditions (3.2) and (3.3) imply (3.10).

Proof. As shown in [COV1], (3.2) holds if and only if there exists a positive constant $c$ such that

$$
\left\|\mathbf{I}_{\alpha} g\right\|_{L^{1+q}\left(\mathbb{R}^{n}, d \sigma\right)} \leq c\|g\|_{L^{p}\left(\mathbb{R}^{n}\right)}, \quad \forall g \in L^{p}\left(\mathbb{R}^{n}\right) .
$$

Since $\mu \in L^{-\alpha, p^{\prime}}\left(\mathbb{R}^{n}\right)$ then $\mathbf{I}_{\alpha} \mu \in L^{p^{\prime}}\left(\mathbb{R}^{n}\right)$. Substituting $g:=\left(\mathbf{I}_{\alpha} \mu\right)^{\frac{1}{p-1}} \in L^{p}\left(\mathbb{R}^{n}\right)$ into (3.11) yields

$$
\left\|\mathbf{I}_{\alpha}\left(\mathbf{I}_{\alpha} \mu\right)^{\frac{1}{p-1}}\right\|_{L^{1+q}\left(\mathbb{R}^{n}, d \sigma\right)} \leq c\left\|\left(\mathbf{I}_{\alpha} \mu\right)^{\frac{1}{p-1}}\right\|_{L^{p}\left(\mathbb{R}^{n}\right)}<+\infty
$$

Notice that $\mathbf{W}_{\alpha, p} \mu$ is always pointwise smaller than $\mathbf{I}_{\alpha}\left(\mathbf{I}_{\alpha} \mu\right)^{\frac{1}{p-1}}$ (see, for example, [Maz, Sec. 10.4.2]). More precisely,

$$
\mathbf{W}_{\alpha, p} \mu \leq C \mathbf{I}_{\alpha}\left(\mathbf{I}_{\alpha} \mu\right)^{\frac{1}{p-1}}
$$

where $C$ is a constant which depends only on $p$ and $n$. This yields (3.10).

The following lemma, in particular, gives necessary conditions for the existence of a positive finite energy solution to equation (1.1). 
Lemma 3.5. Let $1<p<n, 0<q<p-1$ and $\sigma, \mu \in \mathcal{M}^{+}\left(\mathbb{R}^{n}\right)$. Suppose there exists a nontrivial supersolution $u \in L_{\text {loc }}^{q}\left(\mathbb{R}^{n}, d \sigma\right) \cap \dot{W}_{0}^{1, p}\left(\mathbb{R}^{n}\right)$ to equation (1.1). Then

$$
-\Delta_{p} u \in W^{-1, p^{\prime}}\left(\mathbb{R}^{n}\right) \cap \mathcal{M}^{+}\left(\mathbb{R}^{n}\right) \quad \text { and } \quad u \in L^{1+q}\left(\mathbb{R}^{n}, d \sigma\right)
$$

for a quasicontinuous representative of $u$. Consequently, (1.7) and (1.8) hold.

Proof. It follows directly from Lemma 2.7 that

$$
-\Delta_{p} u \in W^{-1, p^{\prime}}\left(\mathbb{R}^{n}\right) \cap \mathcal{M}^{+}\left(\mathbb{R}^{n}\right) \quad \text { and } \quad u \in L^{1+q}\left(\mathbb{R}^{n}, d \sigma\right)
$$

for a quasicontinuous representative of $u$. The former implies (1.8). The latter yields (1.7) in view of the global pointwise lower bound for supersolutions (Theorem 2.4).

In the next theorem, we verify that conditions (1.7) and (1.8) are sufficient for the existstence of a positive finite energy solution to equation (1.1). Further, the minimality of such a solution is also proven.

We first observe that for $1<p<n, 0<\alpha<\frac{n}{p}, \omega, \nu \in \mathcal{M}^{+}\left(\mathbb{R}^{n}\right)$ and $\gamma, \beta \geq 0$,

$$
\mathbf{W}_{\alpha, p}(\gamma \omega+\beta \nu) \leq A\left(\gamma^{\frac{1}{p-1}} \mathbf{W}_{\alpha, p} \omega+\beta^{\frac{1}{p-1}} \mathbf{W}_{\alpha, p} \nu\right)
$$

where $A=A(\alpha, p, n) \geq 1$. This follows immediately from the definition of Wolff's potential and the estimates

$$
|a+b|^{r} \leq \begin{cases}2^{r-1}\left(|a|^{r}+|b|^{r}\right) & \text { for } 1 \leq r<\infty \\ |a|^{r}+|b|^{r} & \text { for } 0 \leq r<1\end{cases}
$$

where $a, b \in \mathbb{R}$.

Theorem 3.6. Let $1<p<n, 0<q<p-1$ and $\sigma, \mu \in \mathcal{M}^{+}\left(\mathbb{R}^{n}\right)$ such that $\sigma \not \equiv 0$. Suppose (1.7) and (1.8) hold. Then there exists a positive finite energy solution $w$ to equation (1.1). Moreover, $w$ is a minimal solution in the sense that $w \leq u$ q.e. (for 
their respective quasicontinuous representatives) for any positive finite energy solution $u$ to $(1.1)$.

Proof. We first prove the existence of $w$. Since (1.7) and (1.8) hold, then by Lemma 3.4, (1.9) holds. By Theorem 3.1 in the case $\alpha=1$, there exists a positive solution $v \in L^{1+q}\left(\mathbb{R}^{n}, d \sigma\right)$ to the integral equation

$$
v=\mathbf{W}_{1, p}\left(v^{q} d \sigma\right)+\mathbf{W}_{1, p} \mu \quad \text { in } \mathbb{R}^{n} .
$$

Using a constant multiple $c^{-1} v$, where $c>0$, in place of $v$, we have

$$
v=c^{\frac{p-1-q}{p-1}} \mathbf{W}_{1, p}\left(v^{q} d \sigma\right)+c \mathbf{W}_{1, p} \mu \quad \text { in } \mathbb{R}^{n} .
$$

Choose $c \geq(K A)^{\frac{p-1}{p-1-q}} \geq K A \geq 1$ where $K \geq 1$ is the constant in Theorem 2.6, and $A \geq 1$ is the constant in (3.12). Then, by Lemma 2.8, we have

$$
v^{q} d \sigma \in W^{-1, p^{\prime}}\left(\mathbb{R}^{n}\right) .
$$

Set

$$
w_{0}:=K^{-1} \mathbf{W}_{1, p} \mu \quad \text { and } \quad d \omega_{0}:=w_{0}^{q} d \sigma+\mu .
$$

Since $K^{-1} \leq 1 \leq c$ then $0<w_{0} \leq v$, and hence

$$
w_{0} \in L^{1+q}\left(\mathbb{R}^{n}, d \sigma\right) \quad \text { and } \quad \omega_{0} \in W^{-1, p^{\prime}}\left(\mathbb{R}^{n}\right) .
$$

As discussed in Remark 2.11, for such a measure $\omega_{0}$, there exists a unique $p$-superharmonic solution $w_{1} \in \dot{W}_{0}^{1, p}\left(\mathbb{R}^{n}\right)$ to the equation

$$
-\Delta_{p} w_{1}=\omega_{0} \quad \text { in } \quad \mathbb{R}^{n} \quad \text { and } \quad\left\|w_{1}\right\|_{\dot{W}_{0}^{1, p}\left(\mathbb{R}^{n}\right)}^{p-1}=\left\|\omega_{0}\right\|_{W^{-1, p}\left(\mathbb{R}^{n}\right)}
$$

for a quasicontinuous representative of $w_{1}$. Moreover, by Theorem 2.6,

$$
0<w_{1} \leq K \mathbf{W}_{1, p} \omega_{0} \leq K A \mathbf{W}_{1, p}\left(v^{q} d \sigma\right)+K A \mathbf{W}_{1, p} f \leq v \quad q . e .
$$


Since $\sigma$ is absolutely continuous with respect to $\operatorname{cap}_{p}$, this yields

$$
w_{1} \in L^{1+q}\left(\mathbb{R}^{n}, d \sigma\right) \quad \text { and } \quad \omega_{1}:=w_{1}^{q} d \sigma+d \mu \in W^{-1, p^{\prime}}\left(\mathbb{R}^{n}\right) .
$$

Again by Theorem 2.6,

$$
w_{0}=K^{-1} \mathbf{W}_{1, p} \mu \leq K^{-1} \mathbf{W}_{1, p} \omega_{0} \leq w_{1} \quad \text { q.e. }
$$

We now have

$$
0<w_{0} \leq w_{1} \leq v \quad \text { q.e. }
$$

We shall construct, by induction, a sequence $\left\{w_{j}\right\}_{j=1}^{\infty}$ so that

$$
\left\{\begin{array}{l}
-\Delta_{p} w_{j}=\sigma w_{j-1}^{q}+\mu \quad \text { in } \mathbb{R}^{n}, \\
w_{j} \in L^{1+q}\left(\mathbb{R}^{n}, d \sigma\right) \cap \dot{W}_{0}^{1, p}\left(\mathbb{R}^{n}\right), \\
\sup _{j \in \mathbb{N}}\left\|w_{j}\right\|_{\dot{W}_{0}^{1, p}\left(\mathbb{R}^{n}\right)}<+\infty \\
w_{j-1}^{q} d \sigma+d \mu \in W^{-1, p^{\prime}}\left(\mathbb{R}^{n}\right), \\
0<w_{j-1} \leq w_{j} \leq v \text { q.e. }
\end{array}\right.
$$

We set

$$
d \omega_{j}:=w_{j}^{q} d \sigma+d \mu, \quad j \in \mathbb{N} .
$$

Suppose $w_{1}, w_{2}, \ldots, w_{j-1}$ have been constructed. Since $\omega_{j-1} \in W^{-1, p^{\prime}}\left(\mathbb{R}^{n}\right)$, then by Remark 2.11, there exists a unique $p$-superharmonic solution $w_{j} \in \dot{W}_{0}^{1, p}\left(\mathbb{R}^{n}\right)$ to the equation

$$
-\Delta_{p} w_{j}=\omega_{j-1} \quad \text { in } \mathbb{R}^{n}
$$

Moreover,

$$
\left\|w_{j}\right\|_{\dot{W}_{0}^{1, p}\left(\mathbb{R}^{n}\right)}^{p}=\left\|\omega_{j-1}\right\|_{W^{-1, p^{\prime}\left(\mathbb{R}^{n}\right)}}^{p^{\prime}} \leq \int_{\mathbb{R}^{n}} w_{j} w_{j-1}^{q} d \sigma+\|\mu\|_{W^{-1, p^{\prime}\left(\mathbb{R}^{n}\right)}}^{p^{\prime}}
$$

Applying Theorem 2.6, we obtain

$$
w_{j} \leq K \mathbf{W}_{1, p} \omega_{j-1} \leq K A \mathbf{W}_{1, p}\left(w_{j-1}^{q} d \sigma\right)+K A \mathbf{W}_{1, p} \mu \quad q . e .
$$


Since $w_{j-1} \leq v$ q.e. then

$$
w_{j} \leq K A \mathbf{W}_{1, p}\left(v^{q} d \sigma\right)+K A \mathbf{W}_{1, p} \mu \leq v \quad q . e .
$$

Hence, $w_{j} \in L^{1+q}\left(\mathbb{R}^{n}, d \sigma\right)$ since $\sigma$ is absolutely continuous with respect to cap p $_{\text {. }}$ Furthermore,

$$
\begin{aligned}
\left\|w_{j}\right\|_{\dot{W}_{0}^{1, p}\left(\mathbb{R}^{n}\right)}^{p} & \leq \int_{\mathbb{R}^{n}} w_{j} w_{j-1}^{q} d \sigma+\|\mu\|_{W^{-1, p^{\prime}}\left(\mathbb{R}^{n}\right)}^{p^{\prime}} \\
& \leq \int_{\mathbb{R}^{n}} v^{1+q} d \sigma+\|\mu\|_{W^{-1, p^{\prime}}\left(\mathbb{R}^{n}\right)}^{p^{\prime}} \\
& <+\infty .
\end{aligned}
$$

This shows that $\left\{w_{j}\right\}_{1}^{\infty}$ is a bounded sequence in $\dot{W}_{0}^{1, p}\left(\mathbb{R}^{n}\right)$. Moreover, since $\omega_{j-2} \leq$ $\omega_{j-1}$, then by Weak Comparison Principle (Lemma 2.9), $w_{j-1} \leq w_{j}$ q.e. Hence, the sequence $\left\{w_{j}\right\}_{j=1}^{\infty}$ satisfying (3.13) has been constructed. Applying the weak continuity of $p$-Laplacian (Theorem 2.3), the Monotone Convergence Theorem and the Weak Compactness Property in $\dot{W}_{0}^{1, p}\left(\mathbb{R}^{n}\right)$, see [HKM, Lemma 1.33], we deduce that the pointwise limit $w:=\lim _{j \rightarrow \infty} w_{j}$ is a positive finite energy solution to (1.1).

We now prove the minimality of $w$. Suppose $u$ is any positive finite energy solution to (1.1). Set $d \omega:=u^{q} d \sigma+d \mu$. By Lemma 3.5, we have

$$
u \in L^{1+q}\left(\mathbb{R}^{n}, d \sigma\right) \quad \text { and } \quad \omega \in W^{-1, p^{\prime}}\left(\mathbb{R}^{n}\right) \cap \mathcal{M}^{+}\left(\mathbb{R}^{n}\right),
$$

for a quasicontinuous representative of $u$. We need to show that $w \leq u$ q.e. Notice that

$$
u \geq\left(\mathbf{W}_{1, p} \mu\right)>K^{-1}\left(\mathbf{W}_{1, p} \mu\right)=w_{0} \quad \text { q.e. }
$$

Therefore $\omega_{0} \leq \omega$ since $\sigma$ is absolutely continuous with respect to cap ${ }_{p}$. By the Weak Comparison Principle (Lemma 2.9), $w_{1} \leq u$ q.e. Arguing by induction as above, we 
see that

$$
w_{j-1} \leq w_{j} \leq u \quad \text { q.e. }
$$

It follows that $w=\lim _{j \rightarrow \infty} w_{j} \leq u$ q.e., which proves the claim.

Remark 3.7. For a similar equation in a domain $\Omega \subset \mathbb{R}^{n}$,

$$
-\Delta_{p} u=u^{q} \sigma+\mu \quad \text { in } \Omega
$$

where $1<p<n, 0<q<p-1$ and $\sigma, \mu \in \mathcal{M}^{+}(\Omega)$, we also have analogous sufficient conditions for the existence of a positive finite energy solution in terms of truncated Wolff's potential, namely:

$$
\mathbf{W}_{1, p}^{R} \sigma \in L^{\frac{(1+q)(p-1)}{p-1-q}}(\Omega, d \sigma), \quad R \geq 2 \operatorname{diam}(\Omega)
$$

and

$$
\mu \in \dot{W}^{-1, p^{\prime}}(\Omega)
$$

Here, for $1<p<\infty, 0<\alpha<\frac{n}{p}$ and $\sigma \in \mathcal{M}^{+}(\Omega)$, the truncated Wolff potential $\mathbf{W}_{\alpha, p}^{R} \sigma$ is defined by (see [KuMi])

$$
\mathbf{W}_{\alpha, p}^{R} \sigma(x)=\int_{0}^{R}\left[\frac{\sigma(B(x, r) \cap \Omega)}{r^{n-\alpha p}}\right]^{\frac{1}{p-1}} \frac{d r}{r}, \quad x \in \Omega, \quad 0<R \leq+\infty .
$$

Moreover, conditions (3.15) and (3.16) are also necessary whenever $\sigma$ and $\mu$ have compact supports in $\Omega$. These results are deduced easily from Theorem 1.1; see details in $[\mathrm{PV}]$.

\subsection{Existence result for equation (1.2)}

In this section, we employ an argument similar to the one used in the previous section to deduce necessary and sufficient conditions for the existence of a positive finite energy solution to fractional Laplace equation (1.2). 
Definition 3.8. Let $q>0,0<\alpha<\frac{n}{2}$ and $\sigma, \mu \in \mathcal{M}^{+}\left(\mathbb{R}^{n}\right)$. A finite energy solution $u$ to equation (1.2) will be understood in the sense that $u \in L_{l o c}^{q}\left(\mathbb{R}^{n}, d \sigma\right) \cap \dot{H}^{\alpha}\left(\mathbb{R}^{n}\right)$, $u \geq 0 \quad d \sigma$-a.e. such that

$$
(-\Delta)^{\frac{\alpha}{2}} u=\mathbf{I}_{\alpha}\left(u^{q} d \sigma\right)+\mathbf{I}_{\alpha} \mu \quad d x \text {-a.e. }
$$

Remark 3.9. Using the same notation as above, suppose $u$ is a positive finite energy solution to (1.2). By mean of definition, we have

$$
(-\Delta)^{\frac{\alpha}{2}} u=\mathbf{I}_{\alpha}\left(u^{q} d \sigma\right)+\mathbf{I}_{\alpha} \mu \quad d x \text {-a.e. }
$$

where $(-\Delta)^{\frac{\alpha}{2}} u=f$ is the unique function in $L^{2}\left(\mathbb{R}^{n}\right)$ such that $u=\mathbf{I}_{\alpha} f$. Therefore

$$
u(x)=\mathbf{I}_{2 \alpha}\left(u^{q} d \sigma\right)(x)+\mathbf{I}_{2 \alpha} \mu(x) \quad \text { whenever } u(x)<+\infty .
$$

Notice that $u \in L_{l o c}^{q}\left(\mathbb{R}^{n}, d \sigma\right) \cap \dot{H}^{\alpha}\left(\mathbb{R}^{n}\right)$. Then

$$
u=\mathbf{I}_{2 \alpha}\left(u^{q} d \sigma\right)+\mathbf{I}_{2 \alpha} \mu \quad d \sigma \text {-a.e. and q.e. }
$$

In particular,

$$
u \geq \mathbf{I}_{2 \alpha}\left(u^{q} d \sigma\right) \quad d \sigma \text {-a.e. }
$$

which implies, by Lemma 2.12 , that $\sigma$ is absolutely continuous with respect to cap ${ }_{\alpha, 2}$. On the other hand, (3.8) implies in particular that

$$
(-\Delta)^{\frac{\alpha}{2}} u \geq \mathbf{I}_{\alpha} \mu \quad d x \text {-a.e. }
$$

Therefore $\mathbf{I}_{\alpha} \mu \in L^{2}\left(\mathbb{R}^{n}\right)$, and hence $\mu \in \dot{H}^{-\alpha}\left(\mathbb{R}^{n}\right)$. In particular, $\mu$ is absolutely continuous with respect to $\operatorname{cap}_{\alpha, 2}$ (see, for example, [AH, Sec. 7]). In summary, $u$ satisfies integral equation (3.18) in any one of the following senses: a.e., $d \sigma$-a.e., $d \mu$-a.e., and q.e. 
The following important observation is analogous to Lemma 3.4.

Lemma 3.10. Let $0<q<1,0<\alpha<\frac{n}{2}$ and $\sigma, \mu \in \mathcal{M}^{+}\left(\mathbb{R}^{n}\right)$. Then (1.13) and (1.14) imply (1.16)

Proof. As shown in [COV1], (1.13) holds if and only if there exists a positive constant $c$ such that

$$
\left\|\mathbf{I}_{\alpha} g\right\|_{L^{1+q\left(\mathbb{R}^{n}, d \sigma\right)}} \leq c\|g\|_{L^{2}\left(\mathbb{R}^{n}\right)}, \quad \forall g \in L^{2}\left(\mathbb{R}^{n}\right) .
$$

Letting $g:=\mathbf{I}_{\alpha} \mu \in L^{2}\left(\mathbb{R}^{n}\right)$ in (3.19), we have

$$
\left\|\mathbf{I}_{2 \alpha} \mu\right\|_{L^{1+q}\left(\mathbb{R}^{n}, d \sigma\right)} \leq c\left\|\mathbf{I}_{\alpha} \mu\right\|_{L^{2}\left(\mathbb{R}^{n}\right)}<+\infty
$$

which proves (1.16).

The neccessary conditions for the existence of a positive finite energy solution to equation (1.2) are established in the following lemma.

Lemma 3.11. Let $0<q<1,0<\alpha<\frac{n}{2}$ and $\sigma, \mu \in \mathcal{M}^{+}\left(\mathbb{R}^{n}\right)$ such that $\sigma \not \equiv 0$. Suppose there exists a positive finite energy solution u to equation (1.2). Then (1.14) holds and $u \in L^{1+q}\left(\mathbb{R}^{n}, d \sigma\right)$. Consequently, (1.13) holds.

Proof. Suppose $u$ is a positive finite energy solution to (1.2). Then (1.14) holds as discussed in Remark 3.9. We next show that $u \in L^{1+q}\left(\mathbb{R}^{n}, d \sigma\right)$. By (3.17), for each nonnegative function $\varphi \in L^{2}\left(\mathbb{R}^{n}\right)$, we have

$$
\int_{\mathbb{R}^{n}}\left[(-\Delta)^{\frac{\alpha}{2}} u\right] \varphi d x=\int_{\mathbb{R}^{n}}\left[\mathbf{I}_{\alpha}\left(u^{q} d \sigma\right)\right] \varphi d x+\int_{\mathbb{R}^{n}}\left[\mathbf{I}_{\alpha} \mu\right] \varphi d x .
$$

Applying Tonelli's Theorem and Schwarz's inequality, we obtain

$$
\begin{aligned}
\left|\int_{\mathbb{R}^{n}} u^{q}\left[\mathbf{I}_{\alpha} \varphi\right] d \sigma\right| & =\left|\int_{\mathbb{R}^{n}}\left[\mathbf{I}_{\alpha}\left(u^{q} d \sigma\right)\right] \varphi d x\right| \\
& \leq\left|\int_{\mathbb{R}^{n}}\left[(-\Delta)^{\frac{\alpha}{2}} u\right] \varphi d x\right|+\left|\int_{\mathbb{R}^{n}}\left(\mathbf{I}_{\alpha} \mu\right) \varphi d x\right| \\
& \leq c\|\varphi\|_{L^{2}\left(\mathbb{R}^{n}\right)},
\end{aligned}
$$


where $c:=\left\|(-\Delta)^{\frac{\alpha}{2}} u\right\|_{L^{2}\left(\mathbb{R}^{n}\right)}+\left\|\mathbf{I}_{\alpha} \mu\right\|_{L^{2}\left(\mathbb{R}^{n}\right)}<\infty$, since $u \in \dot{H}^{\alpha}\left(\mathbb{R}^{n}\right)$ and $\mu \in \dot{H}^{-\alpha}\left(\mathbb{R}^{n}\right)$. Letting $\varphi:=(-\Delta)^{\frac{\alpha}{2}} u$, which is a nonnegative function of class $L^{2}\left(\mathbb{R}^{n}\right)$ in $(3.20)$, we get

$$
\|u\|_{L^{1+q\left(\mathbb{R}^{n}, d \sigma\right)}}^{1+q} \leq c\left\|(-\Delta)^{\frac{\alpha}{2}} u\right\|_{L^{2}\left(\mathbb{R}^{n}\right)}<+\infty
$$

This shows that $u \in L^{1+q}\left(\mathbb{R}^{n}, d \sigma\right)$. Notice that

$$
u=\mathbf{I}_{2 \alpha}\left(u^{q} d \sigma\right)+\mathbf{I}_{2 \alpha} \mu \quad d \sigma-a . e .
$$

Hence, by the discussion in Remark 3.3 in the case $p=2$, we have that (1.13) holds.

The next theorem shows that conditions (1.13) and (1.14) allow us to construct a positive finite energy solution to equation (1.2). Minimality of such a solution will be proven as well.

Theorem 3.12. Let $0<q<1,0<\alpha<\frac{n}{2}$ and $\sigma, \mu \in \mathcal{M}^{+}\left(\mathbb{R}^{n}\right)$. Suppose (1.13) and (1.14) hold. Then there exists a positive finite energy solution $w$ to equation (1.2). Moreover, $w$ is a minimal solution in the sense that $w \leq u$ q.e. for any positive finite energy solution $u$ to (1.2).

Proof. We first prove the existence of $w$. Since (1.13) and (1.14) hold, then by Lemma 3.10 it follows that (1.16) holds. By Theorem 3.1 in the case $p=2$, there exists a positive solution $w \in L^{1+q}\left(\mathbb{R}^{n}, d \sigma\right)$ to the integral equation

$$
w=\mathbf{I}_{2 \alpha}\left(w^{q} d \sigma\right)+\mathbf{I}_{2 \alpha} \mu \quad \text { in } \mathbb{R}^{n} .
$$

We will show that

$$
w \in L_{l o c}^{q}\left(\mathbb{R}^{n}, d \sigma\right) \cap \dot{H}^{\alpha}\left(\mathbb{R}^{n}\right) .
$$


Clearly, $w \in L_{l o c}^{q}\left(\mathbb{R}^{n}, d \sigma\right)$ by Hölder's inequality. In order to prove that $w \in \dot{H}^{\alpha}\left(\mathbb{R}^{n}\right)$, by duality, it suffices to show that there exists a positive constant $c$ such that

$$
\left|\int_{\mathbb{R}^{n}} w \psi d x\right| \leq c\|\psi\|_{\dot{H}^{-\alpha}\left(\mathbb{R}^{n}\right)}, \quad \psi \in C_{0}^{\infty}\left(\mathbb{R}^{n}\right) .
$$

By the semigroup property of the Riesz potentials, Tonelli's Theorem and Hölder's inequality, we have

$$
\begin{aligned}
\left|\int_{\mathbb{R}^{n}} w \psi d x\right| & \leq \int_{\mathbb{R}^{n}} \mathbf{I}_{\alpha}\left(w^{q} d \sigma\right)\left|\mathbf{I}_{\alpha} \psi\right| d x+\int_{\mathbb{R}^{n}} \mathbf{I}_{\alpha} \mu\left|\mathbf{I}_{\alpha} \psi\right| d x \\
& \leq\left\|\mathbf{I}_{\alpha}\left(w^{q} d \sigma\right)\right\|_{L^{2}\left(\mathbb{R}^{n}\right)}\left\|\mathbf{I}_{\alpha} \psi\right\|_{L^{2}\left(\mathbb{R}^{n}\right)}+\left\|\mathbf{I}_{\alpha} \mu\right\|_{L^{2}\left(\mathbb{R}^{n}\right)}\left\|\mathbf{I}_{\alpha} \psi\right\|_{L^{2}\left(\mathbb{R}^{n}\right)} \\
& =\left[\left\|\mathbf{I}_{\alpha}\left(w^{q} d \sigma\right)\right\|_{L^{2}\left(\mathbb{R}^{n}\right)}+\|\mu\|_{\dot{H}^{-\alpha}\left(\mathbb{R}^{n}\right)}\right]\|\psi\|_{\dot{H}^{-\alpha}\left(\mathbb{R}^{n}\right)}
\end{aligned}
$$

for all $\psi \in C_{0}^{\infty}\left(\mathbb{R}^{n}\right)$. Since $\|\mu\|_{\dot{H}^{-\alpha}\left(\mathbb{R}^{n}\right)}<+\infty$, we see that, in view of (3.22) and (3.23), it remains to show that

$$
\left\|\mathbf{I}_{\alpha}\left(w^{q} d \sigma\right)\right\|_{L^{2}\left(\mathbb{R}^{n}\right)}<\infty .
$$

To this end, notice that by the result in [COV1], (1.13) is equivalent to

$$
\left\|\mathbf{I}_{\alpha} g\right\|_{L^{1+q}\left(\mathbb{R}^{n}, d \sigma\right)} \leq c\|g\|_{L^{2}\left(\mathbb{R}^{n}\right)}, \quad \forall g \in L^{2}\left(\mathbb{R}^{n}\right)
$$

where $c$ is a positive constant independent of $g$. Moreover, by duality, (3.25) is equivalent to

$$
\left\|\mathbf{I}_{\alpha}(\varphi d \sigma)\right\|_{L^{2}\left(\mathbb{R}^{n}\right)} \leq c\|\varphi\|_{L^{\frac{1+q}{q}}\left(\mathbb{R}^{n}, d \sigma\right)}, \quad \forall \varphi \in L^{\frac{1+q}{q}}\left(\mathbb{R}^{n}, d \sigma\right)
$$

where $c$ is a positive constant independent of $\varphi$. Letting $\varphi:=w^{q} \in L^{\frac{1+q}{q}}\left(\mathbb{R}^{n}, d \sigma\right)$ in (3.26), we have

$$
\left\|\mathbf{I}_{\alpha}\left(w^{q} d \sigma\right)\right\|_{L^{2}\left(\mathbb{R}^{n}\right)} \leq c\|w\|_{L^{1+q}\left(\mathbb{R}^{n}, d \sigma\right)}^{q}<+\infty
$$

which proves $(3.24)$, and hence $w \in \dot{H}^{\alpha}\left(\mathbb{R}^{n}\right)$. Moreover, by (3.21), we have

$$
(-\Delta)^{\frac{\alpha}{2}} w=\mathbf{I}_{\alpha}\left(w^{q} d \sigma\right)+\mathbf{I}_{\alpha} \mu \quad \text { a.e. }
$$


This shows that $w$ is a positive finite energy solution to (1.2).

Minimality of the solution $w$ is obvious by its construction in Theorem 3.1 in the case $p=2$. Recall that $w$ is the pointwise limit $w=\lim _{j \rightarrow \infty} w_{j}$, where

$$
w_{0}:=\mathbf{I}_{2 \alpha} \mu \quad \text { and } \quad w_{j+1}:=\mathbf{I}_{2 \alpha}\left(w_{j}^{q} d \sigma\right)+\mathbf{I}_{2 \alpha} \mu, \quad j \in \mathbb{N}_{0} .
$$

If $u$ is any positive finite energy solution to (1.2). Then

$$
w_{0}=\mathbf{I}_{2 \alpha} \mu \leq \mathbf{I}_{2 \alpha}\left(u^{q} d \sigma\right)+\mathbf{I}_{2 \alpha} \mu=u \quad \text { q.e. }
$$

Consequently,

$$
w_{1}=\mathbf{I}_{2 \alpha}\left(w_{0}^{q} d \sigma\right)+\mathbf{I}_{2 \alpha} \mu \leq \mathbf{I}_{2 \alpha}\left(u^{q} d \sigma\right)+\mathbf{I}_{2 \alpha} \mu=u \quad \text { q.e. }
$$

Arguing by induction, we obtain

$$
w_{j-1} \leq w_{j} \leq u \text { q.e. for all } j \in \mathbb{N} \text {. }
$$

Therefore, $w=\lim _{j \rightarrow \infty} w_{j} \leq u$ q.e. This proves the minimality of $w$.

\subsection{Existence result for equation (1.3)}

In this section, we establish necessary and sufficient conditions for the existence of a positive finite energy solution to sublinear equation (1.3) on an arbitrary domain which possesses a positive Green function. This proves the existence result stated in Theorem 1.3 in the case $\gamma=1$. Further, it will be used in our proof of characterization

of generalized energy $\mathbb{E}_{\gamma}[u]=\int_{\Omega}|\nabla u|^{2}|u|^{\gamma-1} d x$ in the case $\gamma>1$, see Lemma 4.4 in the next chapter.

Definition 3.13. Let $q>0$ and $\sigma, \mu \in \mathcal{M}^{+}(\Omega)$. Let $G$ be a positive Green function associated with $\mathcal{L}$ on $\Omega$. A solution $u$ to equation (1.3) is understood in the sense 
that $u$ is an $\mathcal{A}$-superharmonic function on $\Omega$ such that $u \in L_{l o c}^{q}(\Omega, d \sigma)$ with $u \geq 0$ $d \sigma$-a.e., and

$$
u=\mathbf{G}\left(u^{q} d \sigma\right)+\mathbf{G} \mu \text { in } \Omega .
$$

If further $u \in \dot{W}_{0}^{1,2}(\Omega)$, it is called a finite energy solution to (1.3).

The following theorem gives necessary and sufficient conditions for the existence of a positive solution $u \in L^{1+q}(\Omega, d \sigma)$ to integral equation (3.28). It is a more general version of Theorem 3.1 in the linear case $p=2$, and will be generalized later in Theorem 5.1.

Theorem 3.14. Let $0<q<1$ and $\sigma, \mu \in \mathcal{M}^{+}(\Omega)$ with $\sigma \not \equiv 0$. Suppose $G$ is a positive quasi-symmetric lower semicontinuous kernel on $\Omega \times \Omega$, which satisfies the WMP. If (1.20) and (1.26) hold with $\gamma=1$, i.e.,

$$
\mathbf{G} \sigma \in L^{\frac{1+q}{1-q}}(\Omega, d \sigma)
$$

and

$$
\mathbf{G} \mu \in L^{1+q}(\Omega, d \sigma),
$$

respectively, then there exists a positive solution $u \in L^{1+q}(\Omega, d \sigma)$ to equation (3.28). The converse statement is valid without the quasi-symmetry assumption on $G$.

Proof. The sufficiency part is similar to the one of Theorem 3.1 when $p=2$, proved by applying Theorem 2.15 (ii) in the case $r=1+q$ in place of Lemma 3.2, and replacing Wolff's potentials by potential operators $\mathbf{G}$ associated with the kernel $G$. The necessity part follows from global pointwise lower bound (2.17):

$$
u(x) \geq c[\mathbf{G} \sigma(x)]^{\frac{1}{1-q}}, \quad \forall x \in \Omega,
$$

which does not require quasi-symmetry of $G$ (cf. Theorem 5.1 in the case $\gamma=1$ ). 
We now apply the above result to deduce necessary and sufficient conditions for the existence of a positive finite energy solution to equation (1.3). As in previous sections, we first make the following observation regarding relation between conditions (3.29), (3.30) and (1.21) in the case $\gamma=1$, i.e.,

$$
\int_{\Omega} \mathbf{G} \mu d \mu<+\infty
$$

or equivalently, $\mu$ has finite energy:

$$
\mu \in \dot{W}^{-1,2}(\Omega)
$$

provided $G$ is a positive Green function associated with $\mathcal{L}$ on $\Omega$.

Lemma 3.15. Let $0<q<1$ and $\sigma, \mu \in \mathcal{M}^{+}(\Omega)$, and let $G$ be a positive lower semicontinuous kernel on $\Omega \times \Omega$, which satisfies the WMP. Then (3.29) and (3.31) imply (3.30).

Proof. See Lemma 5.2 in the case $\gamma=1$.

We would like to mention here that there is an alternative proof of Lemma 3.15 in the particular case when $G$ is a positive Green function associated with $\mathcal{L}=-\Delta$, see [SV1, Lemma 5.3].

The next lemma shows in particular that conditions (3.29) and (3.31) are neccessary for the existence of a positive finite energy solution to equation (1.3).

Lemma 3.16. Let $0<q<1$ and $\sigma, \mu \in \mathcal{M}^{+}(\Omega)$ so that $\sigma \not \equiv 0$, and let $G$ be Green's function associated with $\mathcal{L}$ on $\Omega$. Suppose there exists a positive supersolution $u \in L_{l o c}^{q}(\Omega, d \sigma) \cap \dot{W}_{0}^{1,2}(\Omega)$ to equation (1.3). Then

$$
\mathcal{L} u \in \dot{W}^{-1,2}(\Omega) \cap \mathcal{M}^{+}(\Omega),
$$


and hence (3.31) holds. Moreover, $u \in L^{1+q}(\Omega, d \sigma)$ for a quasicontinuous representative of $u$, and consequently (3.29) holds as well.

Proof. Appealing to the uniform ellipticity condition (1.4) and Schwarz's inequality, for every $\varphi \in C_{0}^{\infty}(\Omega)$ we have

$$
|\langle\mathcal{L} u, \varphi\rangle|=\left|\int_{\Omega} \mathcal{A} \nabla u \cdot \nabla \varphi d x\right| \leq M\|\nabla u\|_{L^{2}(\Omega)}\|\nabla \varphi\|_{L^{2}(\Omega)} .
$$

Hence, $\mathcal{L} u \in \dot{W}^{-1,2}(\Omega)$. Moreover, for every nonnegative $\varphi \in C_{0}^{\infty}(\Omega)$ we have

$$
\langle\mathcal{L} u, \varphi\rangle=\int_{\Omega} \mathcal{A} \nabla u \cdot \nabla \varphi d x \geq \int_{\Omega} u^{q} \varphi d \sigma+\int_{\Omega} \varphi d \mu \geq 0 .
$$

This shows that $\mathcal{L} u \in \mathcal{M}^{+}(\Omega)$, from which it follows that (3.31) holds, and

$$
d \nu:=u^{q} d \sigma \in \dot{W}^{-1,2}(\Omega) \cap \mathcal{M}^{+}(\Omega) .
$$

Let $\left\{\varphi_{j}\right\}_{j=1}^{\infty} \subset C_{0}^{\infty}(\Omega)$ be a sequence of nonnegative functions such that $\varphi_{j} \rightarrow u$ in $\dot{W}_{0}^{1,2}(\Omega)$ as $j \rightarrow \infty$. Then

$$
\left\langle\nu, \varphi_{j}\right\rangle \leq \int_{\Omega} \mathcal{A} \nabla u \cdot \nabla \varphi_{j} d x \quad \text { for all } j \in \mathbb{N}
$$

Hence,

$$
\langle\nu, u\rangle=\lim _{j \rightarrow \infty}\left\langle\nu, \varphi_{j}\right\rangle \leq \lim _{j \rightarrow \infty} \int_{\Omega} \mathcal{A} \nabla u \cdot \nabla \varphi_{j} d x \leq M \int_{\Omega}|\nabla u|^{2} d x<+\infty .
$$

Applying the Brezis-Browder theorem (Theorem 2.10), for a quasicontinuous representative of $u$, we have

$$
\int_{\Omega} u^{1+q} d \sigma=\int_{\Omega} u d \nu=\langle\nu, u\rangle<+\infty
$$

Hence, $u \in L^{1+q}(\Omega, d \sigma)$. Consequently, by Theorem 3.14, (3.29) holds. 
Finally, if $G$ is Green's function associated with $\mathcal{L}$ on $\Omega$, the next lemma shows in particular that conditions (3.29) and (3.31) are sufficient for the existence of a minimal positive finite energy solution to equation (1.3).

Lemma 3.17. Let $0<q<1$ and $\sigma, \mu \in \mathcal{M}^{+}(\Omega)$ so that $\sigma \not \equiv 0$, and let $G$ be a positive Green function associated with $\mathcal{L}$. Suppose (3.29) and (3.31) hold. Then there exists a positive finite energy solution $w \in L_{l o c}^{q}(\Omega, d \sigma) \cap \dot{W}_{0}^{1,2}(\Omega)$ to equation (1.3). Moreover, $w$ is a minimal positive solution in the sense that $w \leq u$ q.e. for any positive solution $u \in L_{l o c}^{q}(\Omega, d \sigma) \cap \dot{W}_{0}^{1,2}(\Omega)$ to equation (1.3).

Proof. Since (3.29) and (3.31) hold, then by Lemma 3.15, it follows that (3.30) holds. In view of Theorem 3.14, there exists a positive solution $w \in L^{1+q}(\Omega, d \sigma)$ to (3.28). Obviously, $w \in L_{l o c}^{q}(\Omega, d \sigma)$ by Hölder's inequality. We will show that $w \in \dot{W}_{0}^{1,2}(\Omega)$. We observe that by the characterization of Green energy discussed in Chapter 4,

$$
\begin{aligned}
\left\|\mathbf{G}\left(w^{q} d \sigma\right)\right\|_{\dot{W}_{0}^{1,2}(\Omega)} & =\int_{\Omega}\left|\nabla \mathbf{G}\left(w^{q} d \sigma\right)\right|^{2} d x \\
& \leq c \int_{\Omega} \mathbf{G}\left(w^{q} d \sigma\right) w^{q} d \sigma \\
& \leq c\left\|\mathbf{G}\left(w^{q} d \sigma\right)\right\|_{L^{1+q}(\Omega, d \sigma)}\left\|w^{q}\right\|_{L^{\frac{1+q}{q}}(\Omega, d \sigma)} \\
& \leq c\|w\|_{L^{1+q}(\Omega, d \sigma)} \\
& <+\infty .
\end{aligned}
$$

Hence

$$
\begin{aligned}
\|w\|_{\dot{W}_{0}^{1,2}(\Omega)} & \leq\left\|\mathbf{G}\left(w^{q} d \sigma\right)\right\|_{\dot{W}_{0}^{1,2}(\Omega)}+\|\mathbf{G} \mu\|_{\dot{W}_{0}^{1,2}(\Omega)} \\
& =\left\|\mathbf{G}\left(w^{q} d \sigma\right)\right\|_{\dot{W}_{0}^{1,2}(\Omega)}+\|\mu\|_{\dot{W}^{-1,2}(\Omega)} \\
& <+\infty
\end{aligned}
$$

which proves the lemma. As in the proof of Theorem 3.12, minimality of $w$ follows immediately from its construction in Theorem 3.14. 


\subsection{Uniqueness results}

In this section, we establish the uniqueness of positive finite energy solutions to equations (1.1), (1.2) and (1.3), using the idea presented in [CV1], namely employing convexity properties of Dirichlet integrals and minimality of such solutions.

Theorem 3.18. Let $1<p<n, 0<q<p-1$ and $\sigma, \mu \in \mathcal{M}^{+}\left(\mathbb{R}^{n}\right)$. Suppose there exists a positive finite energy solution to equation (1.1). Then such a solution is unique in $\dot{W}_{0}^{1, p}\left(\mathbb{R}^{n}\right)$.

Proof. Suppose $u$ and $v$ are positive finite energy solutions to (1.1). We start with the following two observations. We first claim that

$$
\text { if } u=v d \sigma \text {-a.e. then } u=v \text { as elements of } \dot{W}_{0}^{1, p}\left(\mathbb{R}^{n}\right) \text {. }
$$

To see this, suppose $u=v d \sigma$-a.e., and set

$$
d \omega:=u^{q} d \sigma+d \mu=v^{q} d \sigma+d \mu .
$$

Then, $\omega \in \mathcal{M}^{+}\left(\mathbb{R}^{n}\right)$ and

$$
-\Delta_{p} u=-\Delta_{p} v=\omega \quad \text { in } \mathbb{R}^{n}
$$

As usual, we may consider quasicontinuous representatives of $u$ and $v$. Then, by Lemma 3.5,

$$
u, v \in L^{1+q}\left(\mathbb{R}^{n}, d \sigma\right) \quad \text { and } \quad \omega \in W^{-1, p^{\prime}}\left(\mathbb{R}^{n}\right) .
$$

As discussed in Remark 2.11, for such a measure $\omega$, a solution $u \in \dot{W}_{0}^{1, p}\left(\mathbb{R}^{n}\right)$ to the equation $-\Delta_{p} u=\omega$ in $\mathbb{R}^{n}$, is unique. Hence, $u=v$ q.e., so they coincide as elements of $\dot{W}_{0}^{1, p}\left(\mathbb{R}^{n}\right)$.

Secondly, we claim that 


$$
\text { if } u \geq v \text { q.e. then } u=v d \sigma \text {-a.e. }
$$

Suppose $u \geq v$ q.e. then $u \geq v d \sigma$-a.e. and $u \geq v d \mu$-a.e., since $\sigma$ and $\mu$ are absolutely continuous with respect to $\operatorname{cap}_{p}(\cdot)$. Testing the equations

$$
\int_{\mathbb{R}^{n}}|\nabla u|^{p-2} \nabla u \cdot \nabla \phi d x=\int_{\mathbb{R}^{n}} u^{q} \phi d \sigma+\int_{\mathbb{R}^{n}} \phi d \mu, \quad \phi \in \dot{W}_{0}^{1, p}\left(\mathbb{R}^{n}\right),
$$

and

$$
\int_{\mathbb{R}^{n}}|\nabla v|^{p-2} \nabla v \cdot \nabla \psi d x=\int_{\mathbb{R}^{n}} v^{q} \psi d \sigma+\int_{\mathbb{R}^{n}} \psi d \mu, \quad \psi \in \dot{W}_{0}^{1, p}\left(\mathbb{R}^{n}\right),
$$

with $\phi=u$ and $\psi=v$, respectively, where $\omega=u^{q} \sigma+\mu \in \dot{W}^{-1, p^{\prime}}\left(\mathbb{R}^{n}\right)$, so that Theorem 2.10 is applicable for quasi-continuous representatives of $u$ and $v$, we obtain

$$
\int_{\mathbb{R}^{n}}|\nabla u|^{p} d x=\int_{\mathbb{R}^{n}} u^{1+q} d \sigma+\int_{\mathbb{R}^{n}} u d \mu
$$

and

$$
\int_{\mathbb{R}^{n}}|\nabla v|^{p} d x=\int_{\mathbb{R}^{n}} v^{1+q} d \sigma+\int_{\mathbb{R}^{n}} v d \mu .
$$

Using convexity of the Dirichlet integral $\int_{\mathbb{R}^{n}}|\nabla \cdot|^{p} d x$ along curves of the type

$$
\lambda_{t}(x):=\left[(1-t) v^{p}(x)+t u^{p}(x)\right]^{\frac{1}{p}}, \quad t \in[0,1],
$$

see [BF, Proposition 2.6], we obtain

$$
\begin{aligned}
\int_{\mathbb{R}^{n}}\left|\nabla \lambda_{t}\right|^{p} d x & \leq(1-t) \int_{\mathbb{R}^{n}}|\nabla v|^{p} d x+t \int_{\mathbb{R}^{n}}|\nabla u|^{p} d x \\
& =t\left(\int_{\mathbb{R}^{n}}|\nabla u|^{p} d x-\int_{\mathbb{R}^{n}}|\nabla v|^{p} d x\right)+\int_{\mathbb{R}^{n}}|\nabla v|^{p} d x .
\end{aligned}
$$

Notice that $\lambda_{0}=v$. By (3.37) and (3.38), we get

$$
\int_{\mathbb{R}^{n}} \frac{\left|\nabla \lambda_{t}\right|^{p}-\left|\nabla \lambda_{0}\right|^{p}}{t} d x \leq \int_{\mathbb{R}^{n}}\left(u^{1+q}-v^{1+q}\right) d \sigma+\int_{\mathbb{R}^{n}}(u-v) d \mu .
$$

Using the inequality

$$
|a|^{p}-|b|^{p} \geq p|b|^{p-2} b \cdot(a-b) \quad \text { for } a, b \in \mathbb{R}^{n}
$$


we deduce that

$$
\left|\nabla \lambda_{t}\right|^{p}-\left|\nabla \lambda_{0}\right|^{p} \geq p\left|\nabla \lambda_{0}\right|^{p-2} \nabla \lambda_{0} \cdot\left(\nabla \lambda_{t}-\nabla \lambda_{0}\right)
$$

and hence

$$
p \int_{\mathbb{R}^{n}}|\nabla v|^{p-2} \nabla v \cdot \frac{\nabla\left(\lambda_{t}-\lambda_{0}\right)}{t} d x \leq \int_{\mathbb{R}^{n}}\left(u^{1+q}-v^{1+q}\right) d \sigma+\int_{\mathbb{R}^{n}}(u-v) d \mu .
$$

Testing (3.36) with $\psi=\lambda_{t}-\lambda_{0} \in \dot{W}_{0}^{1, p}\left(\mathbb{R}^{n}\right)$, we obtain

$$
\int_{\mathbb{R}^{n}}|\nabla v|^{p-2} \nabla v \cdot \nabla\left(\lambda_{t}-\lambda_{0}\right) d x=\int_{\mathbb{R}^{n}} v^{q}\left(\lambda_{t}-\lambda_{0}\right) d \sigma+\int_{\mathbb{R}^{n}}\left(\lambda_{t}-\lambda_{0}\right) d \mu .
$$

Thus, by (3.39) and (3.40), we have

$$
p \int_{\mathbb{R}^{n}} v^{q} \frac{\lambda_{t}-\lambda_{0}}{t} d \sigma+p \int_{\mathbb{R}^{n}} \frac{\lambda_{t}-\lambda_{0}}{t} d \mu \leq \int_{\mathbb{R}^{n}}\left(u^{1+q}-v^{1+q}\right) d \sigma+\int_{\mathbb{R}^{n}}(u-v) d \mu .
$$

Since $u \geq v$ q.e. then $\lambda_{t} \geq \lambda_{0} d \sigma$-a.e. and $\lambda_{t} \geq \lambda_{0} d \mu$-a.e. Applying Fatou's Lemma, we obtain

$$
\int_{\mathbb{R}^{n}} v^{q} \frac{u^{p}-v^{p}}{v^{p-1}} d \sigma \leq \liminf _{t \rightarrow 0} p \int_{\mathbb{R}^{n}} v^{q} \frac{\lambda_{t}-\lambda_{0}}{t} d \sigma
$$

and

$$
\int_{\mathbb{R}^{n}} \frac{u^{p}-v^{p}}{v^{p-1}} d \mu \leq \liminf _{t \rightarrow 0} p \int_{\mathbb{R}^{n}} \frac{\lambda_{t}-\lambda_{0}}{t} d \mu .
$$

Since (3.41) holds for all $t \in[0,1]$ then by (3.42) and (3.43), we arrive at

$$
\int_{\mathbb{R}^{n}} \frac{u^{p} v^{q}}{v^{p-1}}-v^{1+q} d \sigma+\int_{\mathbb{R}^{n}} \frac{u^{p}}{v^{p-1}}-v d \mu \leq \int_{\mathbb{R}^{n}}\left(u^{1+q}-v^{1+q}\right) d \sigma+\int_{\mathbb{R}^{n}}(u-v) d \mu,
$$

that is,

$$
\int_{\mathbb{R}^{n}} \frac{u^{p} v^{q}}{v^{p-1}}-u^{1+q} d \sigma+\int_{\mathbb{R}^{n}}\left(\frac{u^{p}}{v^{p-1}}-u\right) d \mu \leq 0
$$


Here both integrals on the left-hand side are nonnegative since $u \geq v d \sigma$-a.e. and $u \geq v d \mu$-a.e. Indeed,

$$
\begin{aligned}
\int_{\mathbb{R}^{n}} \frac{u^{p} v^{q}}{v^{p-1}}-u^{1+q} d \sigma & =\int_{\mathbb{R}^{n}} \frac{u^{p} v^{q}-u^{1+q} v^{p-1}}{v^{p-1}} d \sigma \\
& =\int_{\mathbb{R}^{n}} \frac{u^{1+q} v^{q}\left(u^{p-1-q}-v^{p-1-q}\right)}{v^{p-1}} d \sigma \\
& \geq 0
\end{aligned}
$$

and

$$
\int_{\mathbb{R}^{n}}\left(\frac{u^{p}}{v^{p-1}}-u\right) d \mu=\int_{\mathbb{R}^{n}} \frac{u^{p}-u v^{p-1}}{v^{p-1}} d \mu \geq 0 .
$$

Therefore, both integrals must vanish, and thus $u=v d \sigma$-a.e. and $u=v d \mu$-a.e. In particular, this proves the second claim.

Now, suppose $\tilde{w}$ is any positive finite energy solution to (1.1). Then

$$
\tilde{w} \geq w \quad \text { q.e. }
$$

where $w$ is the minimal positive finite energy solution to (1.1) constructed in Theorem 3.6. Applying the second claim above, we have

$$
\tilde{w}=w \quad d \sigma \text {-a.e. }
$$

and hence, by the first claim, they coincide as elements of $\dot{W}_{0}^{1, p}\left(\mathbb{R}^{n}\right)$.

By a slight modification of the argument above, we can establish the uniqueness of a positive finite energy solution to equation (1.2) when $0<\alpha \leq 1$.

Theorem 3.19. Let $0<q<1,0<\alpha \leq 1$, and $\sigma, \mu \in \mathcal{M}^{+}\left(\mathbb{R}^{n}\right)$. Suppose there exists a positive finite energy solution to equation (1.2). Then such a solution is unique in $\dot{H}^{\alpha}\left(\mathbb{R}^{n}\right)$. 
Proof. When $\alpha=1$, this follows from Theorem 3.18 in the case $p=2$. If $0<\alpha<1$, we use the same argument as in the proof of Theorem 3.18 together with convexity of Gagliardo seminorms established in [BF], instead of convexity of the Dirichlet integrals $\int_{\mathbb{R}^{n}}|\nabla \cdot|^{p} d x$.

Using convexity of the Dirichlet integrals $\int_{\Omega}|\nabla \cdot|^{2} d x$ (see $[\mathrm{BF}]$ ), which is comparable to $\int_{\Omega} \mathcal{A} \nabla u \cdot \nabla u d x$ due to the uniform ellipticity condition (1.4), we may argue in the same way as in the proof of Theorem 3.18 in the case $p=2$, together with to obtain the following theorem on the uniqueness of a positive finite energy solution to equation (1.3).

Theorem 3.20. Let $0<q<1$, and let $\sigma, \mu \in \mathcal{M}^{+}(\Omega)$. Suppose there exists a positive finite energy solution to equation (1.3). Then such a solution is unique in $\dot{W}_{0}^{1,2}(\Omega)$. 


\section{Chapter 4}

\section{Generalized energy of measures}

Let $\gamma>0$ and $\omega \in \mathcal{M}^{+}(\Omega)$, and let $G$ be a positive lower semicontinuous kernel on $\Omega \times \Omega$. Define the $\gamma$-energy of $\omega$ by

$$
\mathcal{E}_{\gamma}[\omega]:=\int_{\Omega}(\mathbf{G} \omega)^{\gamma} d \omega
$$

In the case $\gamma=1$, we use the notation $\mathcal{E}[\omega]:=\mathcal{E}_{1}[\omega]$. Observe that $\mathcal{E}_{\gamma}[\omega]$ is welldefined, even though it may be infinite.

When $G$ is a quasi-symmetric kernel on $\Omega \times \Omega$ which satisfies the WMP, by the definition of $\mathcal{E}_{\gamma}[\omega]$ and Theorem 2.15 with $r:=\frac{\gamma(1+\gamma)}{1+\gamma+\gamma^{2}}$ and $q:=\frac{\gamma^{2}}{1+\gamma+\gamma^{2}}$, the following statements are equivalent:

(a) $\mathcal{E}_{\gamma}[\omega]<+\infty$

(b) $\mathbf{G} \omega \in L^{\gamma}(\Omega, d \omega)$.

(c) The weighted norm inequality (2.22) is valid.

(d) There exists a positive solution $u \in L^{r}(\Omega, d \omega)$ to (2.16).

There is also a similar characterization of $\mathcal{E}_{\gamma}[\omega]$, in terms of weak-type and strongtype inequalities, for nondegenerate kernels $G$, see [QV2]. 
We now consider $\mathcal{E}_{\gamma}[\omega]$ in the case where $G$ is a positive Green function associated with $\mathcal{L}$ on $\Omega$.

Theorem 4.1. Let $\gamma>0$ and $\omega \in \mathcal{M}^{+}(\Omega)$ with $\omega \not \equiv 0$, and let $G$ be a positive Green function associated with $\mathcal{L}$ on $\Omega$. If $u:=\mathbf{G} \omega$ then condition

$$
\int_{\Omega}(\mathcal{A} \nabla u \cdot \nabla u) u^{\gamma-1} d x<+\infty
$$

is equivalent to $u^{\frac{\gamma+1}{2}} \in \dot{W}_{0}^{1,2}(\Omega)$ as well as $(a),(b),(c)$ and $(d)$ above. In this case, we have

$$
\mathcal{E}_{\gamma}[\omega]=\gamma \int_{\Omega}(\mathcal{A} \nabla u \cdot \nabla u) u^{\gamma-1} d x
$$

Remark 4.2. By uniform ellipticity condition (1.4), we see that (4.2) is equivalent to (1.5). Therefore, by our discussion above, it suffices to show that

$$
\mathcal{E}_{\gamma}[\omega]<+\infty \Longleftrightarrow \mathbb{E}_{\gamma}[u]<+\infty \Longleftrightarrow u^{\frac{\gamma+1}{2}} \in \dot{W}_{0}^{1,2}(\Omega),
$$

and also establish formula (4.3) whenever $\mathcal{E}_{\gamma}[\omega]<+\infty$.

We first prove an auxiliary fact which will be used in the proof of (4.4) when $0<\gamma<1$

Lemma 4.3. Let $0<\gamma<1$ and $\omega \in \mathcal{M}^{+}(\Omega)$, and let $G$ be a positive Green function associated with $-\mathcal{L}$ on $\Omega$. Suppose $u:=\mathbf{G} \omega \not \equiv \infty$. Then $v:=u^{\gamma}$ is a positive $\mathcal{A}$ superharmonic function on $\Omega$, and $v=\mathbf{G} \mu$, where $\mu \in \mathcal{M}^{+}(\Omega)$ is the Riesz measure of v. Moreover,

$$
\mathcal{E}_{\gamma}[\omega]<+\infty \Longleftrightarrow \mathcal{E}[\mu]<+\infty .
$$

In this case, we have

$$
\frac{\gamma+1}{2} \mathcal{E}_{\gamma}[\omega] \leq \mathcal{E}[\mu] \leq \frac{\gamma+1}{2 \gamma} \mathcal{E}_{\gamma}[\omega]
$$


Proof. Notice that $u:=\mathbf{G} \omega$ is a positive $\mathcal{A}$-superharmonic function on $\Omega$ since $\mathbf{G} \omega \not \equiv$ $+\infty$, see $[\mathrm{GH}]$. Since $0<\gamma<1$, the map $x \longmapsto x^{\gamma}$, for $x \geq 0$, is concave and increasing, it follows that $v:=u^{\gamma}$ is a positive $\mathcal{A}$-superharmonic function on $\Omega$ [HKM, Theorem 7.5]. In light of the Riesz decomposition theorem, $v=\mathbf{G} \mu+h$ where $\mu \in \mathcal{M}^{+}(\Omega)$ is the Riesz measure of $v$, and $h$ is the unique positive $\mathcal{A}$-harmonic function on $\Omega$.

Observe that $g:=h^{\frac{1}{\gamma}}$ is a positive $\mathcal{A}$-subharmonic function on $\Omega$ since $h$ is positive $\mathcal{A}$-harmonic and the map $x \longmapsto x^{\frac{1}{\gamma}}$ for $x \geq 0$, is convex [HKM, Theorem 7.5]. Therefore $-g$ is an $\mathcal{A}$-superharmonic function on $\Omega$, and thus $-g=\mathbf{G} \nu+\tilde{h}$, where $\nu \in \mathcal{M}^{+}(\Omega)$ is the Riesz measure of $-g$, and $\tilde{h}$ is the unique $\mathcal{A}$-harmonic function on $\Omega$. Since

$$
\mathbf{G} \omega=u=v^{\frac{1}{\gamma}}=(\mathbf{G} \mu+h)^{\frac{1}{\gamma}} \geq h^{\frac{1}{\gamma}}=g=-\mathbf{G} \nu-\tilde{h},
$$

we deduce $\mathbf{G}(\omega+\nu)=u+\mathbf{G} \nu \geq-\tilde{h}$. In other words, $-\tilde{h}$ is a positive $\mathcal{A}$-harmonic minorant of the potential $\mathbf{G}(\omega+\nu)$. Consequently, $-\tilde{h}=0$ and thus $g=-\mathbf{G} \nu \leq 0$. This yields $h=g^{\gamma}=0$. We have shown that $v=\mathbf{G} \mu$ is a potential.

Suppose that $\mathcal{E}_{\gamma}[\omega]<+\infty$, and let $w:=u^{\frac{\gamma+1}{2}}$. Since $\frac{\gamma+1}{2} \in(0,1)$, by a similar argument as above, $w$ is a positive $\mathcal{A}$-superharmonic function on $\Omega$, and $w=$ $\mathbf{G} \mu$, where $\mu \in \mathcal{M}^{+}(\Omega)$ is the Riesz measure of $w$. For each $k \in \mathbb{N}$, let $u_{k}:=$ $\min (u, k)$ and $w_{k}:=\min \left(w, k^{\frac{\gamma+1}{2}}\right)$. Using the same argument as above, we see that both $u_{k}$ and $w_{k}$ are potentials with the corresponding Riesz measures $\omega_{k}=\omega\left[u_{k}\right]$ and $\mu_{k}=\mu\left[w_{k}\right]$, respectively. Clearly, $\operatorname{supp}\left(\mu_{k}\right) \subset\{u \leq k\}$, thus both $u_{k}$ and $w_{k}$ are uniformly bounded (by $k$ ) $d \mu_{k}$-a.e. Using Fubini's theorem and the iterated inequality 
(2.19) with $\omega:=\mu_{k}, s:=\frac{2 \gamma}{\gamma+1}$ and $h:=1$, we estimate

$$
\begin{aligned}
\int_{\Omega} \mathbf{G} \mu_{k} d \mu=\int_{\Omega} \mathbf{G} \mu d \mu_{k} & =\int_{\Omega} \mathbf{G} \omega(\mathbf{G} \mu)^{\frac{\gamma-1}{\gamma+1}} d \mu_{k} \\
& \leq \int_{\Omega} \mathbf{G} \omega\left(\mathbf{G} \mu_{k}\right)^{\frac{\gamma-1}{\gamma+1}} d \mu_{k} \\
& =\int_{\Omega} \mathbf{G}\left(\left(\mathbf{G} \mu_{k}\right)^{\frac{\gamma-1}{\gamma+1}} d \mu_{k}\right) d \omega \\
& \leq \frac{\gamma+1}{2 \gamma} \int_{\Omega}\left(\mathbf{G} \mu_{k}\right)^{\frac{2 \gamma}{\gamma+1}} d \omega \\
& \leq \frac{\gamma+1}{2 \gamma} \int_{\Omega}(\mathbf{G} \omega)^{\gamma} d \omega \\
& =\frac{\gamma+1}{2 \gamma} \mathcal{E}_{\gamma}[\omega] .
\end{aligned}
$$

Passing to the limit $k \rightarrow \infty$ and using the monotone convergence theorem, we deduce

$$
\mathcal{E}[\mu]=\int_{\Omega} \mathbf{G} \mu d \mu \leq \frac{\gamma+1}{2 \gamma} \mathcal{E}_{\gamma}[\omega]<+\infty
$$

since $w_{k}=\mathbf{G} \mu_{k} \uparrow w=\mathbf{G} \mu$ in $\Omega$.

Conversely, suppose $\mathcal{E}[\mu]<+\infty$. By using the same notation and argument as above, we estimate

$$
\begin{aligned}
\mathcal{E}[\mu]=\int_{\Omega} \mathbf{G} \mu d \mu=\int_{\Omega}(\mathbf{G} \omega)^{\frac{\gamma+1}{2}} d \mu & \geq \int_{\Omega}\left(\mathbf{G} \omega_{k}\right)^{\frac{\gamma+1}{2}} d \mu \\
& \geq \frac{\gamma+1}{2} \int_{\Omega} \mathbf{G}\left(\left(\mathbf{G} \omega_{k}\right)^{\frac{\gamma-1}{2}} d \omega_{k}\right) d \mu \\
& \geq \frac{\gamma+1}{2} \int_{\Omega} \mathbf{G}\left((\mathbf{G} \omega)^{\frac{\gamma-1}{2}} d \omega_{k}\right) d \mu
\end{aligned}
$$

In the above estimate, we have $u:=\mathbf{G} \omega \leq k$ and $w=\mathbf{G} \mu \leq k d \omega_{k}$-a.e. Applying Fubini's theorem yields

$$
\int_{\Omega} \mathbf{G}\left((\mathbf{G} \omega)^{\frac{\gamma-1}{2}} d \omega_{k}\right) d \mu=\int_{\Omega}(\mathbf{G} \omega)^{\frac{\gamma-1}{2}} \mathbf{G} \mu d \omega_{k}=\int_{\Omega}(\mathbf{G} \omega)^{\gamma} d \omega_{k}
$$

Since $\gamma \in(0,1)$, we have $u^{\gamma}:=(\mathbf{G} \omega)^{\gamma}$ is a potential, that is, $u^{\gamma}=\mathbf{G} \nu$, where $\nu \in \mathcal{M}^{+}(\Omega)$ is the Riesz measure of $u^{\gamma}$. Applying Fubini's theorem and the monotone 
convergence theorem, we have

$$
\int_{\Omega}(\mathbf{G} \omega)^{\gamma} d \omega_{k}=\int_{\Omega} \mathbf{G} \nu d \omega_{k}=\int_{\Omega} \mathbf{G} \omega_{k} d \nu \uparrow \int_{\Omega} \mathbf{G} \omega d \nu
$$

since $\mathbf{G} \omega_{k}=u_{k} \uparrow u=\mathbf{G} \omega$ in $\Omega$ as $k \rightarrow \infty$. Hence,

$$
\frac{\gamma+1}{2} \mathcal{E}_{\gamma}[\omega]=\frac{\gamma+1}{2} \int_{\Omega} \mathbf{G} \nu d \omega=\frac{\gamma+1}{2} \int_{\Omega} \mathbf{G} \omega d \nu \leq \mathcal{E}[\mu]<+\infty
$$

This completes the proof of the lemma.

We now establish (4.4), which yields the first part of Theorem 4.1.

Lemma 4.4. Let $\gamma>0$ and $\omega \in \mathcal{M}^{+}(\Omega)$ with $\omega \neq \equiv 0$, and let $G$ be a positive Green function associated with $\mathcal{L}$ on $\Omega$. If $u:=\mathbf{G} \omega$ then (4.4) holds. In this case, there exists a positive constant $C$ which depends only on $m, M$ and $\gamma$ such that

$$
C^{-1} \mathcal{E}_{\gamma}[\omega] \leq \mathbb{E}_{\gamma}[u] \leq C \mathcal{E}_{\gamma}[\omega]
$$

Proof. Without loss of generality, we may assume that $u \not \equiv+\infty$. It follows that $u$ is a positive $\mathcal{A}$-superharmonic function in $\Omega$. Moreover, $u \in W_{\text {loc }}^{1, p}(\Omega)$ whenever $1 \leq p<\frac{n}{n-1}$, see [HKM]. Consider three cases as follows:

- Case $\gamma=1$. This is completely analogous to the classical result shown in, for example, [L, Theorem 1.20], due to uniform ellipticity assumption (1.4). In this case we further have formula (4.3) using an approximation argument demonstrated below in Lemma 4.5.

- Case $0<\gamma<1$. In light of Lemma 4.3, we have $v:=u^{\frac{\gamma+1}{2}}$ is a positive $\mathcal{A}$ superharmonic function in $\Omega$, and $v=\mathbf{G} \mu$ where $\mu \in \mathcal{M}^{+}(\Omega)$ is the Riesz measure of $v$. Moreover,

$$
\mathcal{E}_{\gamma}[\omega]<+\infty \Longleftrightarrow \mathcal{E}[\mu]<+\infty \Longleftrightarrow v \in \dot{W}_{0}^{1,2}(\Omega)
$$


In this case, we have

$$
\frac{\gamma+1}{2} \mathcal{E}_{\gamma}[\omega] \leq \mathcal{E}[\mu] \leq \frac{\gamma+1}{2 \gamma} \mathcal{E}_{\gamma}[\omega]
$$

On the other hand, notice that $\nabla v=\frac{\gamma+1}{2} u^{\frac{\gamma-1}{2}} \nabla u$ a.e. in $\Omega$. Appealing to the previous case, we deduce

$$
\mathcal{E}[\mu]=\int_{\Omega} \mathcal{A} \nabla v \cdot \nabla v d x \leq M\left(\frac{\gamma+1}{2}\right)^{2} \int_{\Omega}|\nabla u|^{2} u^{\gamma-1} d x
$$

and similarly

$$
\mathcal{E}[\mu]=\int_{\Omega} \mathcal{A} \nabla v \cdot \nabla v d x \geq m\left(\frac{\gamma+1}{2}\right)^{2} \int_{\Omega}|\nabla u|^{2} u^{\gamma-1} d x
$$

This proves both assertions (4.4) and (4.7), respectively.

- Case $\gamma>1$. Suppose that $v:=u^{\frac{\gamma+1}{2}} \in \dot{W}_{0}^{1,2}(\Omega)$. Therefore, $\mathbb{E}_{\gamma}[u]<+\infty$.

For each $k \in \mathbb{N}$, let $u_{k}=\min (u, k)$, which is a positive $\mathcal{A}$-superharmonic function of the class $L^{\infty}(\Omega) \cap W_{l o c}^{1,2}(\Omega)$. Denote the corresponding Riesz measure of each $u_{k}$ by $\omega_{k}:=\omega\left[u_{k}\right]$. Without loss of generality, we may suppose $v$ is quasicontinuous. Applying inequality (2.25) with $\phi:=v$ and $\omega:=\omega_{k}$, we obtain

$$
\int_{\Omega} u^{\gamma} d \omega_{k} \leq \int_{\Omega} v^{2} \frac{d \omega_{k}}{\mathbf{G} \omega_{k}} \leq C \int_{\Omega}|\nabla v|^{2} d x=C\left(\frac{\gamma+1}{2}\right)^{2} \int_{\Omega}|\nabla u|^{2} u^{\gamma-1} d x
$$

On the other hand, by Fubini's theorem and iterated estimate (2.18) with $\omega:=\omega_{k}$ and $s:=\gamma$, we have

$$
\begin{aligned}
\int_{\Omega} u^{\gamma} d \omega_{k}=\int_{\Omega}(\mathbf{G} \omega)^{\gamma-1} \mathbf{G} \omega d \omega_{k} & =\int_{\Omega} \mathbf{G}\left((\mathbf{G} \omega)^{\gamma-1} d \omega_{k}\right) d \omega \\
& \geq \int_{\Omega} \mathbf{G}\left(\left(\mathbf{G} \omega_{k}\right)^{\gamma-1} d \omega_{k}\right) d \omega \\
& \geq \frac{1}{\gamma} \int_{\Omega}\left(\mathbf{G} \omega_{k}\right)^{\gamma} d \omega .
\end{aligned}
$$

Therefore,

$$
\frac{1}{\gamma} \int_{\Omega}\left(\mathbf{G} \omega_{k}\right)^{\gamma} d \omega \leq C\left(\frac{\gamma+1}{2}\right)^{2} \int_{\Omega}|\nabla u|^{2} u^{\gamma-1} d x
$$


Passing to the limit $k \rightarrow \infty$ and using the monotone convergence theorem, we obtain

$$
\mathcal{E}_{\gamma}[\omega]=\int_{\Omega} u^{\gamma} d \omega \leq C \gamma\left(\frac{\gamma+1}{2}\right)^{2} \int_{\Omega}|\nabla u|^{2} u^{\gamma-1} d x<+\infty
$$

since $u_{k}=\mathbf{G} \omega_{k} \uparrow u=\mathbf{G} \omega$ in $\Omega$.

Conversely, suppose $\mathcal{E}_{\gamma}[\omega]<+\infty$. Write $\gamma=\frac{1+q}{1-q}$ where $0<q<1$, and consider the corresponding sublinear elliptic equation

$$
\mathcal{L} w=\omega w^{q} \quad \text { in } \Omega .
$$

Using a similar argument as in the proof of [SV1, Lemma 5.5], there exists a positive finite energy solution $w \in \dot{W}_{0}^{1,2}(\Omega)$ to (4.8), satisfying

$$
\|w\|_{\dot{W}_{0}^{1,2}(\Omega)} \leq c\left(\int_{\Omega}(\mathbf{G} \omega)^{\frac{1+q}{1-q}} d \omega\right)^{\frac{1}{2}}
$$

where $c=c(m, M, q)>0$. As usual, we may assume $w$ is quasicontinuous. By Theorem 2.13, w obeys the lower bound

$$
w \geq(1-q)^{\frac{1}{1-q}}(\mathbf{G} \omega)^{\frac{1}{1-q}} \text { in } \Omega .
$$

Therefore,

$$
v:=u^{\frac{\gamma+1}{2}}=(\mathbf{G} \omega)^{\frac{\gamma+1}{2}} \leq(1-q)^{-\frac{1}{1-q}} w \quad \text { in } \Omega .
$$

From this, we deduce

$$
\begin{aligned}
\int_{\Omega}|\nabla u|^{2} u^{\gamma-1} d x & =\int_{\Omega} v^{2} \frac{|\nabla u|^{2}}{u^{2}} d x \\
& \leq(1-q)^{-\frac{2}{1-q}} \int_{\Omega} w^{2} \frac{|\nabla u|^{2}}{u^{2}} d x
\end{aligned}
$$

Using inequality (2.24) with $\phi:=w$, along with (4.9), we estimate

$$
\int_{\Omega} w^{2} \frac{|\nabla u|^{2}}{u^{2}} d x \leq C\|w\|_{W^{1,2}(\Omega)}^{2} \leq C c^{2} \mathcal{E}_{\gamma}[\omega] .
$$


Hence, by (4.11) and (4.12), we arrive at

$$
\int_{\Omega}|\nabla u|^{2} u^{\gamma-1} d x \leq C c^{2}(1-q)^{-\frac{2}{1-q}} \mathcal{E}_{\gamma}[\omega]<+\infty .
$$

Moreover, for $r=\frac{2 n}{n-2}$ if $n \geq 3$, and any $r<\infty$ if $n=2$, we have that $v \in L^{r}(\Omega)$, since the same is true for $w \in \dot{W}_{0}^{1,2}(\Omega)$. Recall that $\Omega$ is assumed to be a Green domain in the case $n=2$. In other words, $v \in \dot{W}^{1,2}(\Omega)$, the corresponding Sobolev space equipped with the norm $\|v\|_{\dot{W}^{1,2}(\Omega)}=\|\nabla v\|_{L^{2}(\Omega)}+\|v\|_{L^{r}(\Omega)}$. The fact that $v \in \dot{W}_{0}^{1,2}(\Omega)$ follows from the Deny-Lions theorem (see [AH, Sec. 9.12] and the references cited there). Notice that, for $z \in \partial \Omega$, the quasi-limit $\lim _{x \rightarrow z} v(x)=0$ q.e. by (4.10), since the same is true for $w \in \dot{W}_{0}^{1,2}(\Omega)$ by [Kol], Corollary to Theorem 1. This finishes the proof of lemma.

We now complete the proof of Theorem 4.1 by establishing formula (4.3) whenever $\mathcal{E}_{\gamma}[\omega]<+\infty$, using an approximation procedure.

Lemma 4.5. Let $\gamma>0$ and $\omega \in \mathcal{M}^{+}(\Omega)$ with $\omega \neq \equiv 0$, and let $G$ be a positive Green function associated with $\mathcal{L}$ on $\Omega$. If $u:=\mathbf{G} \omega$ then formula (4.3) is valid whenever $\mathcal{E}_{\gamma}[\omega]<+\infty$

Proof. Suppose $\mathcal{E}_{\gamma}[\omega]<+\infty$. Therefore both (4.2) and (1.5) holds, in views of Lemma 4.4 and uniform ellipticity condition (1.4).

For each $k \in \mathbb{N}$, we set $u_{k}=\min (u, k)$. Notice that $u_{k}$ is a positive $\mathcal{A}$-superharmonic function of the class $L^{\infty}(\Omega) \cap W_{l o c}^{1,2}(\Omega)$. Denote the corresponding Riesz measure of $u_{k}$ by $\omega_{k}:=\omega\left[u_{k}\right]$.

Let $\left\{u_{k}^{(j)}\right\}_{j \geq k}$ be a sequence of mollified $u_{k}$, defined on $\Omega_{j}:=\{x \in \Omega: \operatorname{dist}(x, \partial \Omega)>$ 
$\left.\frac{1}{j}\right\}$. Denote $\omega_{k}^{(j)}:=\mathcal{L} u_{k}^{(j)}$. In addition, for $j \geq k$, select $\varphi_{j} \in C_{0}^{\infty}(\Omega)$ so that

$$
0 \leq \varphi_{j} \leq 1, \quad \operatorname{supp} \varphi_{j} \subset \Omega_{j}, \quad \varphi_{j} \uparrow \chi_{\Omega} \text { as } j \rightarrow \infty, \int_{\Omega}\left|\nabla \varphi_{j}\right|^{2} d x \leq \frac{1}{j^{2 \gamma+2}}
$$

Using integration by parts, we obtain

$$
\begin{aligned}
& \gamma \int_{\Omega}\left(\mathcal{A} \nabla u_{k}^{(j)} \cdot \nabla u_{k}^{(j)}\right)\left(u_{k}^{(j)}\right)^{\gamma-1} \varphi_{j} d x=\int_{\Omega} \nabla\left(\left(u_{k}^{(j)}\right)^{\gamma}\right) \cdot \mathcal{A} \nabla u_{k}^{(j)} \varphi_{j} d x \\
& =\int_{\Omega}\left(u_{k}^{(j)}\right)^{\gamma} \varphi_{j} d \omega_{k}^{(j)}-\int_{\Omega}\left(u_{k}^{(j)}\right)^{\gamma}\left(\mathcal{A} \nabla u_{k}^{(j)} \cdot \nabla \varphi_{j}\right) d x .
\end{aligned}
$$

Letting first $j \rightarrow \infty$, and then $k \rightarrow \infty$, we see that

$$
\gamma \int_{\Omega}\left|\nabla u_{k}^{(j)}\right|^{2}\left(u_{k}^{(j)}\right)^{\gamma-1} \varphi_{j} d x \longrightarrow \gamma \int_{\Omega}|\nabla u|^{2} u^{\gamma-1} d x
$$

and

$$
\int_{\Omega}\left(u_{k}^{(j)}\right)^{\gamma} \varphi_{j} d \omega_{k}^{(j)} \longrightarrow \int_{\Omega} u^{\gamma} d \omega
$$

by means of mollification, the Lebesgue dominated convergence theorem, and weak continuity of $\mathcal{L}$ (Theorem 2.3). Moreover, by Schwarz's inequality, the construction of $\varphi_{j}$, and uniform ellipticity condition (1.4), we deduce

$$
\begin{aligned}
\left|\int_{\Omega}\left(u_{k}^{(j)}\right)^{\gamma}\left(\mathcal{A} \nabla u_{k}^{(j)} \cdot \nabla \varphi_{j}\right) d x\right| & \leq M^{\frac{1}{2}}\left(\int_{\Omega}\left|\nabla \varphi_{j}\right|^{2} d x\right)^{\frac{1}{2}}\left(\int_{\Omega}\left|\nabla u_{k}^{(j)}\right|^{2}\left(u_{k}^{(j)}\right)^{2 \gamma} d x\right)^{\frac{1}{2}} \\
& \leq \frac{M^{\frac{1}{2}}}{j^{\gamma+1}}\left(\int_{\Omega}\left|\nabla u_{k}^{(j)}\right|^{2}\left(u_{k}^{(j)}\right)^{\gamma-1}\left(u_{k}^{(j)}\right)^{\gamma+1} d x\right)^{\frac{1}{2}} \\
& \leq \frac{M^{\frac{1}{2}} k^{\frac{\gamma+1}{2}}}{k^{\gamma+1}}\left(\int_{\Omega}|\nabla u|^{2} u^{\gamma-1} d x\right)^{\frac{1}{2}} \\
& =\frac{M^{\frac{1}{2}}}{k^{\frac{\gamma+1}{2}}}\left(\int_{\Omega}|\nabla u|^{2} u^{\gamma-1} d x\right)^{\frac{1}{2}},
\end{aligned}
$$

which converges to zero as $k \rightarrow \infty$. This proves (4.3).

The following lemma shows, in particular, that if $\mathcal{E}_{\gamma}[\omega]<+\infty$ for some $\gamma>0$, then $\omega \in \mathcal{M}_{0}^{+}(\Omega)$. 
Lemma 4.6. Let $\gamma>0$ and $\omega \in \mathcal{M}^{+}(\Omega)$, and let $G$ be a positive Green function associated with $\mathcal{L}$ on $\Omega$. Suppose that $u:=\mathbf{G} \omega \in L_{\text {loc }}^{\gamma}(\Omega, d \mu)$. Then for every compact set $K \subset \Omega$,

$$
\omega(K) \leq[\operatorname{cap}(K)]^{\frac{\gamma}{1+\gamma}}\left(\int_{K} u^{\gamma} d \omega\right)^{\frac{1}{1+\gamma}} .
$$

In particular, $\omega \in \mathcal{M}_{0}^{+}(\Omega)$.

Proof. Let $K$ be a compact subset of $\Omega$. By (2.25), we have

$$
\int_{K} \frac{d \omega}{u} \leq \operatorname{cap}(K)
$$

On the other hand, by Hölder's inequality,

$$
\omega(K)=\int_{K} u^{\frac{-\gamma}{1+\gamma}} u^{\frac{\gamma}{1+\gamma}} d \omega \leq\left(\int_{K} u^{-1} d \omega\right)^{\frac{\gamma}{1+\gamma}}\left(\int_{K} u^{\gamma} d \omega\right)^{\frac{1}{1+\gamma}} .
$$

Thus, (4.13) follows from (4.14) and (4.15). 


\section{Chapter 5}

\section{Solutions with finite generalized energy}

In this chapter, we complete the proof of our main results stated in Theorem 1.3 using the argument outlined earlier in Chapter 1. Its consequences are also discussed here.

\subsection{Existence result for equation (1.3)}

Our first theorem gives necessary and sufficient conditions for the existence of a positive solution $u \in L^{\gamma+q}(\Omega, d \sigma)$ to the integral equation (3.28) in the sublinear case $0<q<1$, under some mild assumptions on kernel $G$ satisfied by the Green function associated with $\mathcal{L}$ on $\Omega$.

Theorem 5.1. Let $0<q<1, \gamma>0$ and $\sigma, \mu \in \mathcal{M}^{+}(\Omega)$ with $\sigma \not \equiv 0$. Suppose $G$ is a positive quasi-symmetric lower semicontinuous kernel on $\Omega \times \Omega$, which satisfies the $W M P$. If (1.20) and (1.26) hold, then there exists a positive solution $u \in L^{\gamma+q}(\Omega, d \sigma)$ to equation (3.28). The converse statement is valid without the quasi-symmetry assumption on $G$.

Proof. Suppose (1.20) and (1.26) hold. In the homogeneous case $\mu \equiv 0$, we can construct a monotone increasing sequence of positive functions $\left\{u_{j}\right\}_{j=0}^{\infty} \subset L^{\gamma+q}(\Omega, d \sigma)$ 
by setting

$$
u_{0}:=\kappa(\mathbf{G} \sigma)^{\frac{1}{1-q}} \quad \text { and } \quad u_{j+1}:=\mathbf{G}\left(u_{j}^{q} d \sigma\right), \quad \text { for } j \in \mathbb{N}_{0}
$$

where $\kappa>0$ is chosen to be sufficiently small. Then its pointwise limit $u:=\lim _{j \rightarrow \infty} u_{j}$ is a positive solution of the class $L^{\gamma+q}(\Omega, d \sigma)$ to (3.28), by the monotone convergence theorem (see [Ver2, Theorem 1.1 (ii)] for more details).

In the inhomogeneous case $\mu \not \equiv 0$, we set

$$
u_{0}:=\mathbf{G} \mu, \quad u_{j+1}:=\mathbf{G}\left(u_{j}^{q} d \sigma\right)+\mathbf{G} \mu, \quad \text { for } j \in \mathbb{N}_{0} .
$$

Observe that $u_{0}>0$ since $\mu \not \equiv 0$, and

$$
u_{1}=\mathbf{G}\left(u_{0}^{q} d \sigma\right)+u_{0} \geq u_{0}
$$

Suppose $u_{0} \leq u_{1} \leq \ldots \leq u_{j}$ for some $j \in \mathbb{N}$. Then

$$
u_{j+1}=\mathbf{G}\left(u_{j}^{q} d \sigma\right)+\mathbf{G} \mu \geq \mathbf{G}\left(u_{j-1}^{q} d \sigma\right)+\mathbf{G} \mu=u_{j}
$$

Hence, by induction, $\left\{u_{j}\right\}_{j=0}^{\infty}$ is an increasing sequence of positive functions. Further, each $u_{j} \in L^{\gamma+q}(\Omega, d \sigma)$. Notice that

$$
u_{0}=\mathbf{G} \mu \in L^{\gamma+q}(\Omega, d \sigma),
$$

by the assumption (1.26). Suppose $u_{0}, \ldots, u_{j} \in L^{\gamma+q}(\Omega, d \sigma)$ for some $j \in \mathbb{N}$. Observe that

$$
\begin{aligned}
\left\|u_{j+1}\right\|_{L^{\gamma+q}(\Omega, d \sigma)} & =\left\|\mathbf{G}\left(u_{j}^{q} d \sigma\right)+\mathbf{G} \mu\right\|_{L^{\gamma+q}(\Omega, d \sigma)} \\
& \leq c\left\|\mathbf{G}\left(u_{j}^{q} d \sigma\right)\right\|_{L^{\gamma+q}(\Omega, d \sigma)}+c\|\mathbf{G} \mu\|_{L^{\gamma+q}(\Omega, d \sigma)},
\end{aligned}
$$

where $c=\max \left(1,2^{\frac{1-\gamma-q}{\gamma+q}}\right)$. In view of Theorem 2.15, the assumption (1.20) is equivalent to the weighted norm inequality (2.22) with $\omega=\sigma$ and $r=\gamma+q$. Therefore, 
we can estimate the first term on the right-hand side of (5.1) by applying (2.22) with $f=u_{j}^{q} \in L^{\frac{\gamma+q}{q}}(\Omega, d \sigma)$,

$$
\begin{aligned}
& \left\|\mathbf{G}\left(u_{j}^{q} d \sigma\right)\right\|_{L^{\gamma+q}(\Omega, d \sigma)} \leq C\left(\int_{\Omega} u_{j}^{\gamma+q} d \sigma\right)^{\frac{q}{\gamma+q}} \\
& \leq C\left(\int_{\Omega} u_{j+1}^{\gamma+q} d \sigma\right)^{\frac{q}{\gamma+q}}=C\left\|u_{j+1}\right\|_{L^{\gamma+q}(\Omega, d \sigma)}^{q} .
\end{aligned}
$$

By (5.1) and (5.2), we have

$$
\left\|u_{j+1}\right\|_{L^{\gamma+q}(\Omega, d \sigma)} \leq C c\left\|u_{j+1}\right\|_{L^{\gamma+q}(\Omega, d \sigma)}^{q}+c\|\mathbf{G} \mu\|_{L^{\gamma+q}(\Omega, d \sigma)^{\prime}} .
$$

We estimate the first term on the right-hand side of (5.3) using Young's inequality,

$$
C c\left\|u_{j+1}\right\|_{L^{\gamma+q}(\Omega, d \sigma)}^{q} \leq q\left\|u_{j+1}\right\|_{L^{\gamma+q}(\Omega, d \sigma)}+(1-q)(C c)^{\frac{1}{1-q}} .
$$

Hence, by (5.3) and (5.4), we obtain

$$
\left\|u_{j+1}\right\|_{L^{\gamma+q}(\Omega, d \sigma)} \leq(C c)^{\frac{1}{1-q}}+\frac{c}{1-q}\|\mathbf{G} \mu\|_{L^{\gamma+q}(\Omega, d \sigma)}<+\infty .
$$

By induction, we have shown that each $u_{j} \in L^{\gamma+q}(\Omega, d \sigma)$. Finally, applying the monotone convergence theorem to the sequence $\left\{u_{j}\right\}_{j=0}^{\infty}$, we see that the pointwise limit $u:=\lim _{j \rightarrow \infty} u_{j}$ exists so that $u>0, u \in L^{\gamma+q}(\Omega, d \sigma)$, and satisfies (3.28).

Conversely, if there exists a positive solution $u \in L^{\gamma+q}(\Omega, d \sigma)$ to (3.28), it is clear that (1.26) holds. Moreover, (1.20) follows from the global pointwise lower bound (2.17):

$$
u(x) \geq c[\mathbf{G} \sigma(x)]^{\frac{1}{1-q}}, \quad \forall x \in \Omega,
$$

which does not require quasi-symmetry of $G$ (see $[\mathrm{GV}]$ ).

An essential link between conditions (1.20), (1.21) and (1.26), will be obtained in the next lemma. It is an extension of Lemma 3.15 in the case $\gamma=1$. 
Lemma 5.2. Let $0<q<1, \gamma>0$, and $\sigma, \mu \in \mathcal{M}^{+}(\Omega)$. Suppose $G$ is a positive lower semicontinuous kernel on $\Omega \times \Omega$, which satisfies the WMP. Then conditions (1.20) and (1.21) imply (1.26).

Proof. Without loss of generality, we may assume $\sigma, \mu \not \equiv 0$. Consider the following three cases:

- Case 1: $\gamma+q>1$. Applying the iterated inequality (2.18) with $\omega=\mu$ and $s=\gamma+q$, together with Fubini's theorem and Hölder's inequality with the exponents $\frac{\gamma}{\gamma+q-1}$ and $\frac{\gamma}{1-q}$, we obtain

$$
\begin{aligned}
\int_{\Omega}(\mathbf{G} \mu)^{\gamma+q} d \sigma & \leq c \int_{\Omega} \mathbf{G}\left((\mathbf{G} \mu)^{\gamma+q-1} d \mu\right) d \sigma \\
& =c \int_{\Omega}(\mathbf{G} \mu)^{\gamma+q-1} \mathbf{G} \sigma d \mu \\
& \leq c\left[\int_{\Omega}(\mathbf{G} \mu)^{\gamma} d \mu\right]^{\frac{\gamma+q-1}{\gamma}}\left[\int_{\Omega}(\mathbf{G} \sigma)^{\frac{\gamma}{1-q}} d \mu\right]^{\frac{1-q}{\gamma}} .
\end{aligned}
$$

The second integral on the right-hand side of (5.6) is estimated by a similar argument as above. In fact, applying (2.18) again with $\omega=\sigma$ and $s=\frac{\gamma}{1-q}$, along with Fubini's theorem and Hölder's inequality with the exponents $\frac{\gamma+q}{\gamma+q-1}$ and $\gamma+q$, we deduce

$$
\begin{aligned}
\int_{\Omega}(\mathbf{G} \sigma)^{\frac{\gamma}{1-q}} d \mu & \leq c \int_{\Omega} \mathbf{G}\left((\mathbf{G} \sigma)^{\frac{\gamma}{1-q}-1} d \sigma\right) d \mu \\
& =c \int_{\Omega}(\mathbf{G} \sigma)^{\frac{\gamma+q-1}{1-q}} \mathbf{G} \mu d \sigma \\
& \leq c\left[\int_{\Omega}(\mathbf{G} \sigma)^{\frac{\gamma+q}{1-q}} d \sigma\right]^{\frac{\gamma+q-1}{\gamma+q}}\left[\int_{\Omega}(\mathbf{G} \mu)^{\gamma+q} d \sigma\right]^{\frac{1}{\gamma+q}} .
\end{aligned}
$$

By (5.6) and (5.7), we have

$$
\left[\int_{\Omega}(\mathbf{G} \mu)^{\gamma+q} d \sigma\right]^{1-\frac{1-q}{\gamma(\gamma+q)}} \leq c\left[\int_{\Omega}(\mathbf{G} \mu)^{\gamma} d \mu\right]^{\frac{\gamma+q-1}{\gamma}}\left[\int_{\Omega}(\mathbf{G} \sigma)^{\frac{\gamma+q}{1-q}} d \sigma\right]^{\frac{(\gamma+q-1)(1-q)}{\gamma(\gamma+q)}},
$$

which is finite by (1.20) and (1.21), and hence (1.26) holds.

- Case 2: $\gamma+q<1$. Write

$$
\int_{\Omega}(\mathbf{G} \mu)^{\gamma+q} d \sigma=\int_{\Omega}(\mathbf{G} \mu)^{\gamma+q} F^{a-1} F^{1-a} d \sigma
$$


where $0<a<1$ and $F$ is a positive measurable function to be determined later. Applying Hölder's inequality with the exponents $\frac{1}{a}$ and $\frac{1}{1-a}$, we get

$$
\int_{\Omega}(\mathbf{G} \mu)^{\gamma+q} d \sigma \leq\left(\int_{\Omega}(\mathbf{G} \mu)^{\frac{\gamma+q}{a}} F^{\frac{a-1}{a}} d \sigma\right)^{a}\left(\int_{\Omega} F d \sigma\right)^{1-a} .
$$

Setting $F=(\mathbf{G} \sigma)^{\frac{\gamma+q}{1-q}}$ and $a=\gamma+q$ in (5.9), we obtain

$$
\int_{\Omega}(\mathbf{G} \mu)^{\gamma+q} d \sigma \leq\left(\int_{\Omega} \mathbf{G} \mu(\mathbf{G} \sigma)^{\frac{\gamma+q-1}{\gamma+q}} d \sigma\right)^{\gamma+q}\left(\int_{\Omega}(\mathbf{G} \sigma)^{\frac{\gamma+q}{1-q}} d \sigma\right)^{1-\gamma-q}
$$

The first integral on the right-hand side of (5.10) is estimated by using Fubini's theorem, followed by inequality (2.19) with $\omega=\sigma$ and $s=\frac{\gamma}{1-q}$,

$$
\begin{aligned}
\int_{\Omega} \mathbf{G} \mu(\mathbf{G} \sigma)^{\frac{\gamma+q-1}{\gamma+q}} d \sigma & =\int_{\Omega} \mathbf{G}\left((\mathbf{G} \sigma)^{\frac{\gamma+q-1}{\gamma+q}} d \sigma\right) d \mu \\
& \leq c \int_{\Omega}(\mathbf{G} \sigma)^{\frac{\gamma}{1-q}} d \mu .
\end{aligned}
$$

As above, we deduce

$$
\begin{aligned}
\int_{\Omega}(\mathbf{G} \sigma)^{\frac{\gamma}{1-q}} d \mu & \leq\left(\int_{\Omega} \mathbf{G} \sigma(\mathbf{G} \mu)^{\gamma+q-1} d \mu\right)^{\frac{\gamma}{1-q}}\left(\int_{\Omega}(\mathbf{G} \mu)^{\gamma} d \mu\right)^{\frac{1-\gamma-q}{1-q}} \\
& =\left(\int_{\Omega} \mathbf{G}\left((\mathbf{G} \mu)^{\gamma+q-1} d \mu\right) d \sigma\right)^{\frac{\gamma}{1-q}}\left(\int_{\Omega}(\mathbf{G} \mu)^{\gamma} d \mu\right)^{\frac{1-\gamma-q}{1-q}} \\
& \leq c\left(\int_{\Omega}(\mathbf{G} \mu)^{\gamma+q} d \sigma\right)^{\frac{\gamma}{1-q}}\left(\int_{\Omega}(\mathbf{G} \mu)^{\gamma} d \mu\right)^{\frac{1-\gamma-q}{1-q}} .
\end{aligned}
$$

Combining the preceding estimates, we have

$$
\left(\int_{\Omega}(\mathbf{G} \mu)^{\gamma+q} d \sigma\right)^{1-\frac{\gamma(\gamma+q)}{1-q}} \leq c\left[\int_{\Omega}(\mathbf{G} \mu)^{\gamma} d \mu\right]^{\frac{(1-\gamma-q)(\gamma+q)}{1-q}}\left[\int_{\Omega}(\mathbf{G} \sigma)^{\frac{\gamma+q}{1-q}} d \sigma\right]^{1-\gamma-q}
$$

This proves (1.26), since both integrals on the right-hand side of (5.12) are finite by (1.20) and (1.21).

- Case 3: $\gamma+q=1$. Fix a positive number $\frac{1}{2-q}<a<1$. Applying Hölder's 
inequality with the exponents $\frac{1}{a}$ and $\frac{1}{1-a}$ we have

$$
\begin{aligned}
\int_{\Omega} \mathbf{G} \mu d \sigma & =\int_{\Omega} \mathbf{G} \mu(\mathbf{G} \sigma)^{\frac{a-1}{1-q}}(\mathbf{G} \sigma)^{\frac{1-a}{1-q}} d \sigma \\
& \leq\left(\int_{\Omega}(\mathbf{G} \mu)^{\frac{1}{a}}(\mathbf{G} \sigma)^{\frac{a-1}{a(1-q)}} d \sigma\right)^{a}\left(\int_{\Omega}(\mathbf{G} \sigma)^{\frac{1}{1-q}} d \sigma\right)^{1-a} .
\end{aligned}
$$

We estimate the first integral on the right-hand side of (5.13) using inequalities (2.18) and (2.19), together with Fubini's theorem and Hölder's inequality with the exponents $\frac{a(1-q)}{1-a}$ and $\frac{a(1-q)}{a(2-q)-1}$,

$$
\begin{aligned}
\int_{\Omega}(\mathbf{G} \mu)^{\frac{1}{a}}(\mathbf{G} \sigma)^{\frac{a-1}{a(1-q)}} d \sigma & \leq c \int_{\Omega} \mathbf{G}\left[(\mathbf{G} \mu)^{\frac{1-a}{a}} d \mu\right](\mathbf{G} \sigma)^{\frac{a-1}{a(1-q)}} d \sigma \\
& =c \int_{\Omega}(\mathbf{G} \mu)^{\frac{1-a}{a}} \mathbf{G}\left((\mathbf{G} \sigma)^{\frac{a-1}{a(1-q)}} d \sigma\right) d \mu \\
& \leq c \int_{\Omega}(\mathbf{G} \mu)^{\frac{1-a}{a}}(\mathbf{G} \sigma)^{\frac{a-1}{a(1-q)}+1} d \mu \\
& \leq c\left(\int_{\Omega}(\mathbf{G} \mu)^{1-q} d \mu\right)^{\frac{1-a}{a(1-q)}}\left(\int_{\Omega} \mathbf{G} \sigma d \mu\right)^{\frac{a(2-q)-1}{a(1-q)}} \\
& =c\left(\int_{\Omega}(\mathbf{G} \mu)^{1-q} d \mu\right)^{\frac{1-a}{a(1-q)}}\left(\int_{\Omega} \mathbf{G} \mu d \sigma\right)^{\frac{a(2-q)-1}{a(1-q)}} .
\end{aligned}
$$

Combining the preceding estimates, we deduce

$$
\left(\int_{\Omega} \mathbf{G} \mu d \sigma\right)^{1-\frac{a(2-q)-1}{(1-q)}} \leq c\left(\int_{\Omega}(\mathbf{G} \mu)^{1-q} d \mu\right)^{\frac{1-a}{(1-q)}}\left(\int_{\Omega}(\mathbf{G} \sigma)^{\frac{1}{1-q}} d \sigma\right)^{1-a}
$$

which is finite by (1.20) and (1.21). Thus (1.26) holds.

We are now prepared to show that conditions (1.20) and (1.21) are necessary and sufficient for the existence of a positive solution $u \in L^{\gamma+q}(\Omega, d \sigma) \cap L^{\gamma}(\Omega, d \mu)$ to integral equation (3.28), under the same restrictions on the kernel $G$ as above.

Theorem 5.3. Let $0<q<1, \gamma>0$, and let $\sigma, \mu \in \mathcal{M}^{+}(\Omega)$ with $\sigma \not \equiv 0$. Suppose $G$ is a positive, lower semicontinuous, quasi-symmetric kernel on $\Omega \times \Omega$, which satisfies the WMP. Suppose (1.20) and (1.21) hold. Then there exists a positive solution 
$u \in L^{\gamma+q}(\Omega, d \sigma) \cap L^{\gamma}(\Omega, d \mu)$ to (3.28). The converse statement is also valid without the quasi-symmetry assumption on $G$.

Proof. Suppose (1.20) and (1.21) hold. Then, by Lemma 5.2, we see that (1.26) holds. Thus, in light of Theorem 5.1, there exists a positive solution $u \in L^{\gamma+q}(\Omega, d \sigma)$ to (3.28). We will show that $u \in L^{\gamma}(\Omega, d \mu)$ as well. By (1.21), it suffices to establish

$$
\int_{\Omega}\left[\mathbf{G}\left(u^{q} d \sigma\right)\right]^{\gamma} d \mu<+\infty
$$

Without loss of generality, we may assume that $G$ is symmetric, and $\mu \not \equiv 0$. Consider the following two cases:

- Case 1: $\gamma \geq 1$. Applying (2.19) with $\omega:=\sigma u^{q}$ and $s:=\gamma$, along with Fubini's theorem, and Hölder's inequality with the exponents $\frac{\gamma+q}{\gamma+q-1}$ and $\gamma+q$, we have

$$
\begin{aligned}
\int_{\Omega}\left[\mathbf{G}\left(u^{q} d \sigma\right)\right]^{\gamma} d \mu & \leq c \int_{\Omega} \mathbf{G}\left(\left(\mathbf{G}\left(u^{q} d \sigma\right)\right)^{\gamma-1} u^{q} d \sigma\right) d \mu \\
& =c \int_{\Omega}\left(\mathbf{G}\left(u^{q} d \sigma\right)\right)^{\gamma-1}(\mathbf{G} \mu) u^{q} d \sigma \\
& \leq c\left[\int_{\Omega}\left(\mathbf{G}\left(u^{q} d \sigma\right)\right)^{\frac{(\gamma-1)(\gamma+q)}{\gamma+q-1}} u^{\frac{q(\gamma+q)}{\gamma+q-1}} d \sigma\right]^{\frac{\gamma+q-1}{\gamma+q}}\left[\int_{\Omega}(\mathbf{G} \mu)^{\gamma+q} d \sigma\right]^{\frac{1}{\gamma+q}} \\
& \leq c\left[\int_{\Omega} u^{\gamma+q} d \sigma\right]^{\frac{\gamma+q-1}{\gamma+q}}\left[\int_{\Omega}(\mathbf{G} \mu)^{\gamma+q} d \sigma\right]^{\frac{1}{\gamma+q}} \\
& \leq c \int_{\Omega} u^{\gamma+q} d \sigma<+\infty
\end{aligned}
$$

- Case 2: $0<\gamma<1$. We write

$$
\int_{\Omega}\left[\mathbf{G}\left(u^{q} d \sigma\right)\right]^{\gamma} d \mu=\int_{\Omega}\left[\mathbf{G}\left(u^{q} d \sigma\right)\right]^{\gamma} F^{a-1} F^{1-a} d \mu
$$

where $0<a<1$ and $F$ is a positive measurable function to be determined later. Applying Hölder's inequality with the conjugate exponents $\frac{1}{a}$ and $\frac{1}{1-a}$ yields

$$
\int_{\Omega}\left[\mathbf{G}\left(u^{q} d \sigma\right)\right]^{\gamma} d \mu \leq\left[\int_{\Omega} \mathbf{G}\left(u^{q} d \sigma\right) F^{\frac{a-1}{a}} d \mu\right]^{a}\left[\int_{\Omega} F d \mu\right]^{1-a} .
$$


Setting $F:=(\mathbf{G} \mu)^{\gamma}$ and $a:=\gamma$ in $(5.16)$, we get

$$
\int_{\Omega}\left[\mathbf{G}\left(u^{q} d \sigma\right)\right]^{\gamma} d \mu \leq\left[\int_{\Omega} \mathbf{G}\left(u^{q} d \sigma\right)(\mathbf{G} \mu)^{\gamma-1} d \mu\right]^{\gamma}\left[\int_{\Omega}(\mathbf{G} \mu)^{\gamma} d \mu\right]^{1-\gamma} .
$$

We estimate the first integral on the right-hand side of (5.17) using Fubini's theorem, followed by inequality (2.19) with $\omega=\mu$ and $s=\gamma$,

$$
\begin{aligned}
\int_{\Omega} \mathbf{G}\left(u^{q} d \sigma\right)(\mathbf{G} \mu)^{\gamma-1} d \mu & =\int_{\Omega} \mathbf{G}\left((\mathbf{G} \mu)^{r-q-1} d \mu\right) u^{q} d \sigma \\
& \leq c \int_{\Omega}(\mathbf{G} \mu)^{\gamma} u^{q} d \sigma \\
& \leq c \int_{\Omega} u^{\gamma+q} d \sigma<+\infty
\end{aligned}
$$

By (5.17) and (5.18), together with (1.21), this proves (5.15).

Conversely, since $u \in L^{\gamma}(\Omega, d \mu)$, it is clear that (1.21) holds. Further, by Theorem 5.1, condition (1.20) is valid since $u \in L^{\gamma+q}(\Omega, d \sigma)$.

As an application of the preceding theorem, when $G$ is a positive Green function associated with $\mathcal{L}$ in $\Omega$, we deduce the first part of Theorem 1.3 by appealing to the characterization of the generalized Green energy obtained in Chapter 4.

Theorem 5.4. Let $0<q<1, \gamma>0$, and let $\sigma, \mu \in \mathcal{M}^{+}(\Omega)$ with $\sigma \not \equiv 0$. Let $G$ be a positive Green function associated with $\mathcal{L}$ on $\Omega$. Then there exists a positive solution $u \in L_{l o c}^{q}(\Omega, d \sigma)$ to (1.3) satisfying (1.5) if and only if (1.20) and (1.21) hold.

In this case, $u$ is a minimal solution in the sense that $u \leq v$ q.e. for any positive solution $v \in L_{l o c}^{q}(\Omega)$ to (1.3) which satisfies (1.5).

Proof. This follows from Theorem 4.1 with $\omega:=u^{q} d \sigma+\mu$, together with Theorem 5.3. Moreover, arguing by induction, we see that minimality of such a solution follows immediately from its construction in Theorem 5.1 (cf. Lemma 3.17). 


\subsection{Proofs of Corollaries 1.4 and $\mathbf{1 . 5}$}

Applying the next theorem with $\omega:=u^{q} d \sigma+\mu$ yields Corollary 1.4.

Theorem 5.5. Let $n \geq 3$, and $\omega \in \mathcal{M}^{+}(\Omega)$ with $\omega \not \equiv 0$. Let $G$ be a positive Green function associated with $\mathcal{L}$ on $\Omega$. Suppose that $u:=\mathbf{G} \omega$ satisfies (1.5) for some $0<\gamma \leq 1$. Then $u \in \dot{W}_{0}^{1, p}(\Omega)$ where $p=\frac{n(1+\gamma)}{n+\gamma-1}$. If, in addition, $|\Omega|<+\infty$, then the assertion is valid for $1 \leq p \leq \frac{n(1+\gamma)}{n+\gamma-1}$.

Proof. The classical case $\gamma=1$ is known, so we can assume $\gamma \in(0,1)$. Observe that $u$ is a positive $\mathcal{A}$-superharmonic function with zero boundary values, and $p=\frac{n(1+\gamma)}{n+\gamma-1} \in\left(\frac{n}{n-1}, 2\right)$. For each $k \in \mathbb{N}$, set $u_{k}=\min (u, k)$, which is a positive $\mathcal{A}$ superharmonic function of the class $L^{\infty}(\Omega) \cap W_{l o c}^{1,2}(\Omega)$. Let $\left\{\Omega_{k}\right\}_{k=1}^{\infty}$ be an increasing sequence of relatively compact open subsets of $\Omega$ such that $\Omega=\bigcup_{k=1}^{\infty} \Omega_{k}$. Applying Hölder's inequality with the exponents $\frac{2}{p}$ and $\frac{2}{2-p}$, followed by Sobolev's inequality [MZ, Theorem 1.56], we obtain

$$
\begin{aligned}
& \left\|\left(\nabla u_{k}\right) \chi_{\Omega_{k}}\right\|_{L^{p}(\Omega)}^{p}=\int_{\Omega_{k}}\left|\nabla u_{k}\right|^{p} d x \\
& =\int_{\Omega_{k}}\left|\nabla u_{k}\right|^{p} u_{k}^{\frac{(\gamma-1) p}{2}} u_{k}^{\frac{(1-\gamma) p}{2}} d x \\
& \leq\left(\int_{\Omega_{k}}\left|\nabla u_{k}\right|^{2} u_{k}^{\gamma-1} d x\right)^{\frac{p}{2}}\left(\int_{\Omega_{k}} u_{k}^{\frac{(1-\gamma) p}{2-p}} d x\right)^{\frac{2-p}{2}} \\
& \leq\left(\int_{\Omega}|\nabla u|^{2} u^{\gamma-1} d x\right)^{\frac{p}{2}}\left\|u_{k}\right\|_{L^{\frac{(1-\gamma) p}{2-p}}\left(\Omega_{k}\right)}^{\frac{(1-\gamma) p}{2}}\left\|\nabla u_{k}\right\|_{L^{p}\left(\Omega_{k}\right)}^{\frac{(1-\gamma) p}{2}}, \\
& \leq c\left(\int_{\Omega}|\nabla u|^{2} u^{\gamma-1} d x\right)^{\frac{p}{2}}
\end{aligned}
$$

that is,

$$
\left\|\left(\nabla u_{k}\right) \chi_{\Omega_{k}}\right\|_{L^{p}(\Omega)}^{p-\frac{(1-\gamma) p}{2}} \leq c\left(\int_{\Omega}|\nabla u|^{2} u^{\gamma-1} d x\right)^{\frac{p}{2}},
$$

where $c$ is a positive constant independent of $k$. Since $p>\frac{(1-\gamma) p}{2}$, letting $k \rightarrow \infty$ 
in (5.19) yields the assertion by the monotone convergence theorem. This proves $u \in \dot{W}_{0}^{1, p}(\Omega)$, which is obviously true for all $1 \leq p \leq \frac{n(1+\gamma)}{n+\gamma-1}$ when $|\Omega|<+\infty$.

The next proposition shows in particular that a pair of conditions in (1.23) is sufficient for both (1.20) and (1.21).

Proposition 5.6. Let $G$ be a positive lower semicontinuous kernel on $\Omega \times \Omega$ which satisfies

$$
G(x, y) \leq c I_{2 \alpha}(x-y), \quad \forall x, y \in \Omega,
$$

where $I_{2 \alpha}(\cdot)=|\cdot|^{2 \alpha-n}$ is the Riesz kernel of order $2 \alpha\left(0<\alpha<\frac{n}{2}\right)$ on $\mathbb{R}^{n}$, and $c$ is a positive constant. Let $\beta>0$. If $\omega \in L^{s}(\Omega)$ is a positive function, where $s=\frac{n(\beta+1)}{n+2 \alpha \beta}$, then

$$
\mathbf{G} \omega \in L^{\beta}(\Omega, d \omega) .
$$

Proof. Observe that $s=\frac{n(\beta+1)}{n+2 \alpha \beta}>\frac{n(\beta+1)}{n+n \beta}=1$. Then

$$
\int_{\Omega}(\mathbf{G} \omega)^{\beta} d \omega \leq\left(\int_{\Omega}(\mathbf{G} \omega)^{\beta s^{\prime}} d x\right)^{\frac{1}{s^{\prime}}}\|\omega\|_{L^{s}(\Omega)}
$$

where $s^{\prime}=\frac{s}{s-1}$ is the Hölder conjugate of $s$. Denote $\tilde{\omega}$ the zero extension of $\omega$ to $\mathbb{R}^{n}$. By (5.20) and the Hardy-Littlewood-Sobolev inequality, there is a positive constant $C \geq c$ such that

$$
\|\mathbf{G} \omega\|_{L^{\beta s^{\prime}}(\Omega)} \leq C\left\|\mathbf{I}_{2 \alpha} \tilde{\omega}\right\|_{L^{\beta s^{\prime}}\left(\mathbb{R}^{n}\right)} \leq C\|\tilde{\omega}\|_{L^{s}\left(\mathbb{R}^{n}\right)}=C\|\omega\|_{L^{s}(\Omega)} .
$$

Combining (5.22) and (5.23) yields (5.21).

Proposition 5.6 shows that (1.24) implies (1.22). Hence, Corollary 1.5 follows from Corollary 1.4 . 


\section{Bibliography}

[AH] D. R. Adams And L. I. Hedberg, Function Spaces and Potential Theory, Grundlehren der math. Wissenschaften 314, Springer, BerlinHeidelberg-New York, 1996.

[An] A. Ancona, Some results and examples about the behavior of harmonic functions and Green's functions with respect to second order elliptic operators, Nagoya Math. J. 165 (2002), 123-158.

[BB] P. BÉnilan And H. Brezis, Nonlinear problems related to the ThomasFermi equation, J. Evol. Equ. 3 (2003), no. 4, 673-770.

[BO] L. Boccardo And L. Orsina, Sublinear equations in $L^{s}$, Houston J. Math. 20 (1994), 99-114.

[BF] L. Brasco and G. Franzina, Convexity properties of Dirichlet integrals and Picone-type inequalities, Kodai Math. J. 37 (2014), 769-799.

[Br] M. Brelot, Lectures on Potential Theory, Lectures on Mathematics 19, Tata Institute of Fundamental Research, Bombay, 1960.

[BrB] H. Brezis And F. E. Browder, A property of Sobolev spaces, Commun. PDE 44 (1979), 1077-1083.

[BK] H. Brezis and S. Kamin, Sublinear elliptic equations on $\mathbb{R}^{n}$, Manuscr. Math. 74 (1992), 87-106.

[CV1] D. T. CaO and I. E. Verbitsky, Finite energy solutions of quasilinear elliptic equations with sub-natural growth terms, Calc. Var. PDE 52 (2015), $529-546$.

[CV2] D. T. CAO AND I. E. Verbitsky, Nonlinear elliptic equations and intrinsic potentials of Wolff type, J. Funct. Anal. 272 (2017), 112-165.

[COV1] C. Cascante, J. M. Ortega, and I. E. Verbitsky, Trace inequalities of Sobolev type in the upper triangular case, Proc. London Math. Soc. 80 (2000), 391-414.

[COV2] C. Cascante, J. M. Ortega, and I. E. Verbitsky, On $L^{p}-L^{q}$ trace inequalities, J. London Math. Soc. 74 (2006), 497-511. 
[GH] A. GRIGOR'YAN And W. HANSEn, Lower estimates for a perturbed Green function, J. Anal. Math. 104 (2008), 25-58.

[GV] A. Grigor'yan And I. E. Verbitsky, Pointwise estimates of solutions to nonlinear equations for nonlocal operators, to appear in Ann. Sc. Norm. Super. Pisa, arXiv: 1707.09596.

[HW] L. I. HedBerg AND T. WolfF, Thin sets in nonlinear potential theory, Ann. Inst. Fourier (Grenoble) 33 (1983), 161-187.

[HKM] J. Heinonen, T. Kilpeläinen, and O. Martio, Nonlinear Potential Theory of Degenerate Elliptic Equations, Dover Publications, 2006 (unabridged republ. of 1993 edition, Oxford University Press).

[JMV] B. J. JAYE, V. G. MAZ'YA, AND I. E. Verbitsky, Existence and regularity of positive solutions of elliptic equations of Schrödinger type, J. Anal. Math. 118 (2012), 577-621.

[K] C. E. Kenig, Harmonic Analysis Techniques for Second Order Elliptic Boundary Value Problems, CBMS Reg. Conf. Ser. Math. 83, American Mathematical Society, Providence, RI, 1994.

[KKT] T. KilpeläInen, T. Kuusi, and A. Tuhola-Kujanpä̈̈, Superharmonic functions are locally renormalized solutions, Ann. Inst. H. Poincaré, Anal. Non Linéaire 28 (2011), 775-795.

[KM1] T. KILPeläInen AND J. MalÝ, Degenerate elliptic equations with measure data and nonlinear potentials, Ann. Scuola Norm. Super. Pisa, Cl. Sci. 19 (1992), 591-613.

[KM2] T. KILPELÄINEN AND J. MALÝ, The Wiener test and potential estimates for quasilinear elliptic equations, Acta Math. 172 (1994), 137-161.

[Kol] T. KolsRud, A uniqueness theorem for higher order elliptic partial differential equations, Math. Scand. 51 (1982), 323-332.

[KuMi] T. KuUsi And G. Mingione, Guide to nonlinear potential estimates, Bull. Math. Sci. 4 (2014), 1-82.

[L] N. S. LAndkof, Foundations of Modern Potential Theory, Grundlehren der math. Wissenschaften 180, Springer, New York-Heidelberg, 1972.

[Li] P. L. Lions, On the existence of positive solutions of semilinear elliptic equations, SIAM Rev. 24 (1982), 441-467.

[LSW] W. Littman, G. Stampacchia, and H. F. Weinberger Regular points for elliptic equations with discontinuous coefficients, Ann. Sc. Norm. Super. Pisa 17 (1963), 43-77. 
[MZ] J. MalÝ And W. ZIEmer, Fine Regularity of Solutions of Elliptic Partial Differential Equations, Math. Surveys Monogr. 51, Amer. Math. Soc., Providence, RI, 1997.

[Maz] V. MAZ'YA, Sobolev Spaces, with Applications to Elliptic Partial Differential Equations, 2nd revised augm. ed., Grundlehren der math. Wissenschaften 342, Springer, Berlin, 1997.

[MV] V. G. MAZ'YA AND I. E. VerBitsky, The Schrödinger operator on the energy space: boundedness and compactness criteria, Acta Math. 188 (2002), 263-302.

[MS] G. Metafune And C. Spina, An integration by parts formula in Sobolev spaces, Mediterr. J. Math. 5 (2008), 357-369.

[PV] N. C. Phuc And I. E. Verbitsky, Quasilinear and Hessian equations of Lane-Emden type, Ann. Math. 168 (2008), 859-914.

[P] A. C. Ponce, Elliptic PDEs, Measures and Capacities. From the Poisson Equation to Nonlinear Thomas-Fermi Problems, EMS Tracts in Mathematics 23, European Mathematical Society (EMS), Zürich, Switzerland, 2016

[QV1] S. QUINn AND I. E. VERBITSKY, Weighted norm inequalities of $(1, q)$-type for integral and fractional maximal operators, Harmonic Analysis, Partial Differential Equations and Applications, in Honor of Richard L. Wheeden, eds. S. Chanillo et al., Birkhäuser, Ser. Appl. Numer. Harmonic Anal. (2017), 217-238.

[QV2] S. QUINn AND I. E. VerBITsky, A sublinear version of Schur's lemma and elliptic PDE, Analysis \& PDE 11 (2018), 439-466.

[SV1] A. Seesanea And I. E. Verbitsky, Finite energy solutions to inhomogeneous nonlinear elliptic equations with sub-natural growth terms, Adv. Calc. Var. (2017), DOI: 10.1515/acv-2017-0035, arXiv:1709.02048.

[SV2] A. Seesanea And I. E. Verbitsky, Solutions to sublinear elliptic equations with finite generalized energy (submitted), arXiv:1804.09255.

[St] E. M. STEIN Singular Integrals and Differentiability Properties of Functions, Princteon University Press, Princeton, NJ, 1970.

[TW] N. S. Trudinger And X. J. WAng, On the weak continuity of elliptic operators and application to potential theory, Amer. J. Math. 124 (2002), 369-410.

[Vaz] J. L. VÁzquez The Porous Medium Equation, Oxford Mathematical Monographs, The Clarendon Press, Oxford University Press, Oxford, 2007. 
[Ver1] I. E. Verbitsky, Nonlinear potentials and trace inequalities, Oper. Theory: Adv. Appl. 110 (1999), 323-343.

[Ver2] I. E. Verbitsky, Sublinear equations and Schur's test for integral operators, 50 Years with Hardy Spaces, a Tribute to Victor Havin, Oper. Theory: Adv. Appl. 261 (2018), 467-484. 
Adisak Seesanea was born on February 7, 1988 in Ratchaburi, Thailand. He attended Silpakorn University and graduated with a first class honored Bachelor's degree in Mathematics in 2010. After receiving his Master's degree in Applied Mathematics from Suranaree University of Technology in 2013, he came to the United States and began his doctoral study in Mathematics at the University of Missouri. Upon completion of his degree in July 2018, he will take a Research Fellow position at Hokkaido University in Japan. 